\title{
The Evolution of Stellar Populations in the Outer Disks of Spiral Galaxies $^{1}$
}

\author{
Stacey Alberts ${ }^{2}$, Daniela Calzetti ${ }^{2}$, Hui Dong ${ }^{2}$, L. C. Johnson ${ }^{3}$, Daniel A. Dale ${ }^{4}$, Luciana \\ Bianchi $^{5}$, Rupali Chandar ${ }^{6}$, Robert C. Kennicutt ${ }^{7}$, Gerhardt R. Meurer ${ }^{8}$, Michael Regan $^{9}$, \\ David Thilker ${ }^{5}$
}

\begin{abstract}
We investigate recent star formation in the extended ultraviolet (XUV) disks of five nearby galaxies (NGC 0628, NGC 2090, NGC 2841, NGC 3621, and NGC 5055) using a long wavelength baseline comprised of ultraviolet and mid-infrared imaging from the Galaxy Evolution Explorer and the Spitzer Infrared Array Camera. We identify 229 unresolved stellar complexes across targeted portions of their XUV disks and utilize spectral energy distribution fitting to measure their stellar ages and masses through comparison with Starburst99 population synthesis models of instantaneous burst populations. We find that the median age of outer disk associations in our sample is $\sim 100$ Myr with a large dispersion that spans the entire range of our models (1 Myr-1 Gyr). This relatively evolved state for most associations addresses the observed dearth of $\mathrm{H} \alpha$ emission in some outer disks, as $\mathrm{H} \alpha$ can only be observed in star forming regions younger than $~ 10$ Myr. The large age dispersion is robust against variations in extinction (in the range $E(B-V)=0-0.3 \mathrm{mag})$ and variations in the upper end of the stellar Initial Mass Function (IMF). In particular, we demonstrate that the age dispersion is insensitive to steepening of the IMF, up to extreme slopes.
\end{abstract}

Subject headings: Galaxies:spiral — galaxies:star clusters — stars:evolution

\footnotetext{
${ }^{1}$ Based on observations obtained with the Spitzer Space Telescope as part of the Cycle 3 GO program 30753 .

${ }^{2}$ Department of Astronomy, LGRT-B 619E, University of Massachusetts, Amherst, MA 01003, USA

${ }^{3}$ Department of Astronomy, University of Washington, Seattle, WA 98195, USA

${ }^{4}$ Department of Physics and Astronomy, University of Wyoming, Laramie, WY 82071, USA

${ }^{5}$ Department of Physics and Astronomy, Johns Hopkins University, Baltimore, MD 21218, USA

${ }^{6}$ Department of Physics and Astronomy, University of Toledo, Toledo, OH 43606, USA

${ }^{7}$ Institute of Astronomy, Cambridge University, Cambridge CB3 0HA, UK

${ }^{8}$ International Centre for Radio Astronomy, University of Western Australia, Perth, WA 6009, AUS

${ }^{9}$ Space Telescope Science Institute, Baltimore, MD 21218, USA
} 


\section{Introduction}

The discovery of in-situ star formation (SF) in the extended ultraviolet (XUV) disks of spiral galaxies (Thilker et al. 2007) offers us a chance to study the processes of star formation in extreme low-density environments. As the driving force behind the formation and evolution of galactic disks, star formation has been extensively studied in the high-density 'inner' disk. Extending this understanding with studies in the outer disk will have many far-reaching implications. Are we in the presence of a different mode of star formation relative to that of the inner disks? Is the stellar Initial Mass Function (IMF) observed in inner disks applicable to outer disk environments? Can we confirm or challenge current models or scaling relations such as the Schmidt-Kennicutt law (Kennicutt 1998b) or the classical SF threshold (Kennicutt 1989; Martin \& Kennicutt 2001)? What effects do the feedback processes from massive stars in the outer disks have on, and are they a primary component in, the chemical enrichment of the pristine halo? Star formation in the XUV disks of nearby galaxies can be studied in great detail and, as SF is found to occur later in the outer disk than the inner disk (Mun̈oz-Mateos et al. 2007; Roskar et al. 2008), it may provide a similar environment to the low-density, low-metallicity, pristine conditions of newly forming galaxies in the early Universe. In addition, the long term implications of galaxy interactions may still be imprinted on these XUV disks.

The possibility of star formation in the outer disks of galaxies was first revealed by the presence of $\mathrm{H} \alpha$ emission out beyond the galaxies' optical radii (Ferguson et al. 1998; Lelievre \& Roy 2000) and by the discovery of B stars in the outer disk of M31 (Cuillandre et al. 2001). This was confirmed by the Galaxy Evolution Explorer (GALEX), which discovered smooth ultraviolet (UV) profiles that extended well beyond $R_{25}$ in a number of galaxies (Thilker et al. 2005; Gil de Paz et al. 2005; Boissier et al. 2007). This was quickly followed by a study of about 200 S0-Sm galaxies from the GALEX Nearby Galaxies Survey (NGS, e.g. Bianchi et al. (2003, 2009) and references therein; Gil de Paz et al. (2007b)), with corollary optical-NIR imaging by Thilker et al. (2007), who found XUV disks in $\sim 30 \%$ of local $(<40 \mathrm{Mpc})$ galaxies and grouped them into two major types (see, also, Zaritsky \& Christlein $(2007)$ ). These disks are characterized by the presence of many UV-bright knots (Thilker et al. 2005; Gil de Paz et al. 2005), which have since been associated with HI structures, display metallicities in the range of $\mathrm{Z} / \mathrm{Z}_{\odot}=1 / 10-1$ (Ferguson et al. 1998; Gil de Paz et al. 2007a; Bresolin et al. 2009; (Werk et al. 2010), and have been determined to be locales of star forming activity.

As star formation tracers $\mathrm{H} \alpha$, far-ultraviolet (FUV), and near-ultraviolet (NUV) emission all tell us about different populations (Kennicutt 1998a). $\mathrm{H} \alpha$ traces the ionizing population ( $<10 \mathrm{Myr}$ ), while the UV is composed of emission from all young stars, with FUV sensitive to the youngest phases of SF and NUV to a slightly more evolved population ( $\sim 100$ 
Myr). It has been found that the UV emission is more extended than the $\mathrm{H} \alpha$ emission in about half of XUV disks, with the remaining half showing roughly equal extent for UV and $\mathrm{H} \alpha$ (Goddard et al. 2010). The cases of M83 and NGC 4625 demonstrate this dearth of $\mathrm{H} \alpha$ emission, with only $\sim 10-20 \%$ of UV clumps showing associated $\mathrm{H} \alpha$ (Thilker et al. 2005) and, in general, the $\mathrm{H} \alpha$ spatial covering fraction recovered in these XUV disks is about half that seen in the UV (Goddard et al. 2010). We address two of the possible explanations that have been put forth: (1) the star forming associations in these regions exist at a wide range of ages with a majority of associations beyond the age where ionizing stars still exist ( 10 Myr) (Thilker et al. 2005; Zaritsky \& Christlein 2007; Goddard et al. 2010) or (2) in low-density regions high mass star formation is effectively suppressed via a top-light IMF (Meurer et al. 2009). These two solutions are generally difficult to separate due to the ageIMF degeneracy. In this paper, we aim to determine the age range and age distribution of star forming associations in the outer disks of five galaxies assuming a standard Kroupa IMF (Kroupa et al. 2001) and utilizing a long wavelength baseline by combining GALEX far-ultraviolet and near-ultraviolet imaging with Spitzer mid-infrared (MIR; 3.6-8.0 $\mu \mathrm{m}$ ) bands. Then we will perform the same analysis using a top-light IMF and will outline the effects of steepening the IMF slope on the age/mass distribution of our associations. The advantage of our approach, relative to previous studies, is that we do not rely on the presence of ionized gas to assess the existence of IMF variations; we instead exploit the long wavelength baseline afforded by the combination of GALEX and Spitzer data to break the age-IMF degeneracy.

In Section 2, we provide a brief description of how we selected our galaxies and the data used in this paper. Section 3 outlines the process of association identification and selection as well as our photometry. In Section 4, we discuss our models and how they are applied to the data. In Section 5, we present the statistical results per galaxy of the age/mass distribution and, in Section 6, the analysis of how the IMF affects this distribution. Section 7 concludes with a brief outline of future work.

\section{Galaxy Selection, Observations, and Data Reduction}

A recent study of M83 has demonstrated the effectiveness of combining the UV data from GALEX with the MIR bands of the Spitzer Infrared Array Camera (IRAC) to constrain the ages and masses of star forming associations (Dong et al. 2008). To expand on this study, five

galaxies from the GALEX Atlas of Nearby Galaxies (Gil de Paz et al. 2007b) were chosen as a sub-sample of XUV disks that represent the full range in UV morphologies. These different morphologies have been hypothesised to be different phases of disk building (Thilker et al. 2007) and could indicate some influence of the parent galaxy on outer disk populations. Target regions beyond 1.5 $R_{\text {opt }}$ were then chosen for Spitzer and imaged using IRAC's four 
bands: $3.6 \mu \mathrm{m}, 4.5 \mu \mathrm{m}, 5.8 \mu \mathrm{m}$ and $8.0 \mu \mathrm{m}$. These data join the FUV $(\sim 1529 \AA)$ and NUV $(\sim 2312 \stackrel{\circ}{A})$ bands from GALEX.

The five galaxies, NGC 0628, NGC 2090, NGC 2841, NGC 3621, and NGC 5055, can be seen in Figure 1: Table 1 contains their relevant information. These galaxies were selected to be observed with Spitzer because they are close enough $(6-12 \mathrm{Mpc})$ that the IRAC resolution of $\sim 2$ ", which corresponds to a physical scale of 60-110 pc, is larger than the typical size of a compact stellar cluster $(\sim 2-5 \mathrm{pc})$, but not much larger than the typical size of a scaled OB association, $\sim 50$ pc (Maiz-Apellaniz 2001). The outer disk regions targeted with Spitzer were selected to have concentrated UV emission within the central $\sim 1$ arcminute of the $\sim 5$ arcminute Spitzer IRAC field of view in order to maximize the number of detections in the infrared.

As discussed in Thilker et al. (2007), there are two types of XUV disks. Type 1 disks have an extended region of UV-bright and optically-faint complexes that lay beyond the classical threshold for star formation in low-density environments. These disks are represented here by NGC 0628, NGC 2841, NGC 3621, and NGC 5055. NGC 2090 is a Type 2 disk, which is defined as having an extensive outer region within the SF threshold with optically low surface brightness and a blue (FUV-NIR) color.

Table 2 contains the locations of the target regions (also displayed in Figure 1), as well as galaxy-specific exposure times and sensitivities. For the GALEX FUV and NUV filters (Morrissey et al. 2007), the exposure times range from $1644 \mathrm{~s}$ for NGC 0628 to $7269 \mathrm{~s}$ for NGC 3621, as compared to 1500 s integrations for NGS. These correspond to a 3 sigma FUV sensitivity, corrected for Galactic extinction, of $3.46 \times 10^{-18}$ and $2.0 \times 10^{-18} \mathrm{erg} \mathrm{s}^{-1} \mathrm{~cm}^{-2}$ $\AA^{-1}$, respectively.

For the infrared observations, the Spitzer IRAC instrument (Fazio et al. 2004) observed the outer disk fields as part of GO Program 30753 (PI: D. Calzetti). The fields were observed using two separate Astronomical Observing Requests (AORs) that each consisted of nine or ten 100 second dithered integrations (30 seconds for NGC 3621). The dithered imaging allows for accurate removal of cosmic rays and detector artifacts, while executing the

Table 1. Galaxy Information

\begin{tabular}{lcccc}
\hline \hline & $\begin{array}{c}\text { RA } \\
(\mathrm{J} 2000)\end{array}$ & $\begin{array}{c}\text { Dec } \\
(\mathrm{J} 2000)\end{array}$ & $\begin{array}{c}\text { Distance } \\
(\mathrm{Mpc})\end{array}$ & $\begin{array}{c}\text { XUV-Disk } \\
\text { Type }\end{array}$ \\
\hline NGC 0628 & 013641.77 & +154700.50 & 7.3 & $\mathrm{I}$ \\
NGC 2090 & 054701.89 & -341502.20 & 11.2 & $\mathrm{II}$ \\
NGC 2841 & 092202.63 & +505835.47 & 9.8 & $\mathrm{I}$ \\
NGC 3621 & 111816.51 & -324850.60 & 6.6 & $\mathrm{I}$ \\
NGC 5055 & 131549.33 & +420145.40 & 8.3 & $\mathrm{I}$ \\
\hline
\end{tabular}




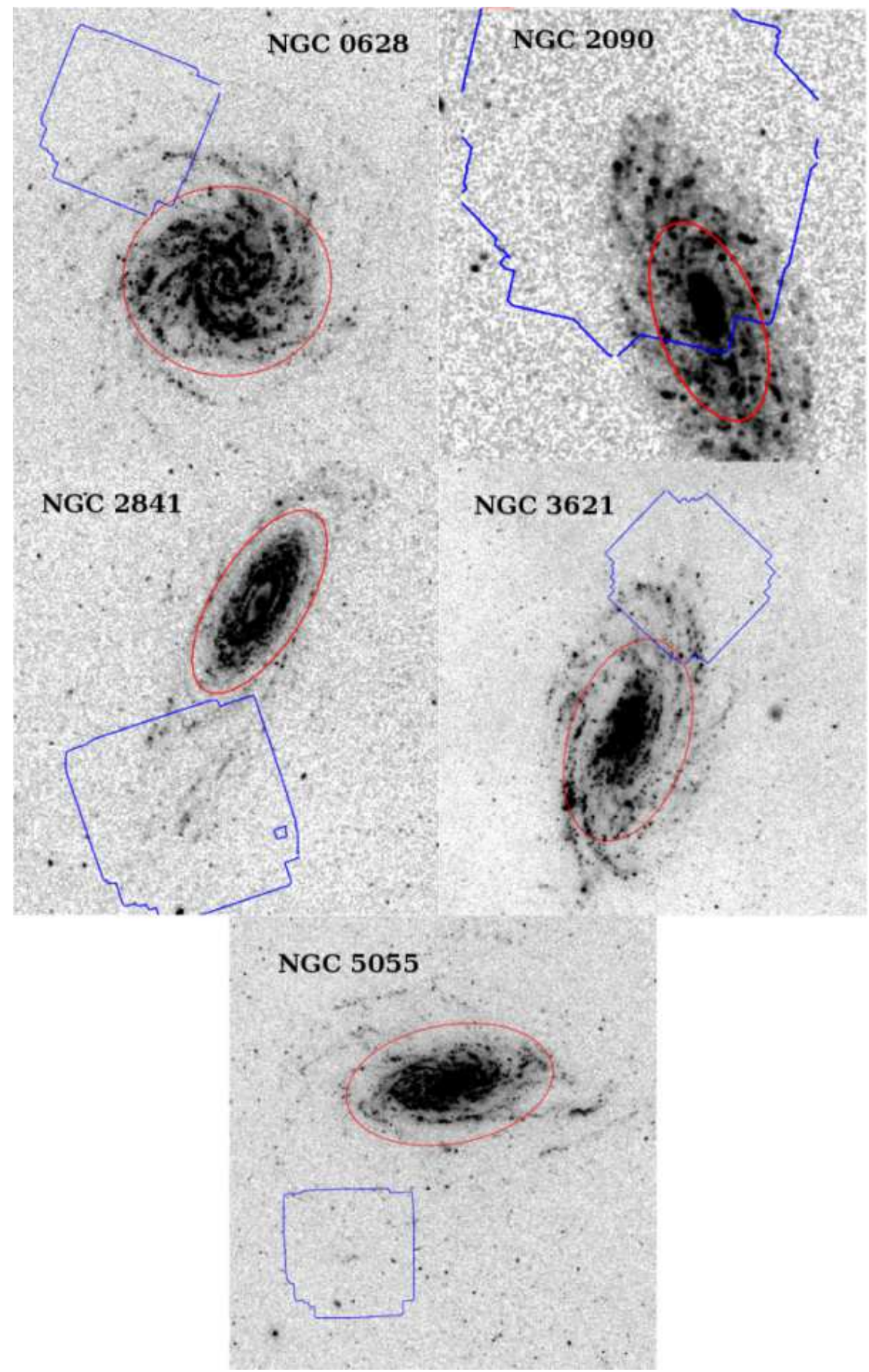

Fig. 1. - The GALEX FUV images for the five galaxies in this study. The red circle traces the $R_{25}$ boundary and the blue box shows the target region imaged with Spitzer/IRAC. 
two AORs separately enables asteroid removal and image redundancy. Imaging data were initially processed by the S14.4 version of the Spitzer Science Center IRAC Basic Calibrated Data (BCD) pipeline. Post-processing image refinement was performed using the Local Volume Legacy (LVL; Dale et al. (2009)) imaging pipeline, which includes artifact correction, mosaicing, and sky subtraction. For this study, the IRAC images were resampled, conserving surface brightness, from an original pixel scale of 0.75 " to match the GALEX pixel scale of 1.5". All four IRAC images and the GALEX FUV images were aligned and registered to the appropriate GALEX NUV image. The final IRAC mosaics have total integration times of $\geq 1800 \mathrm{~s}$ per pixel, yielding an average 3 sigma $3.6 \mu \mathrm{m}$ sensitivity of $8.98 \times 10^{-21} \mathrm{erg} \mathrm{s}^{-1}$ $\mathrm{cm}^{-2} \stackrel{\circ}{A}^{-1}$. Total integration times and sensitivities for each galaxy are provided in Table 2 .

\section{Association Identification and Selection}

Given the resolution of our images, the star forming associations we seek to identify will appear as UV-bright point sources. Following the procedures of Dong et al. (2008), we identify all $2 \sigma$ sources in the FUV image using SExtractor (version 2.4.4, Bertin, E., \& Arnouts, S. (1996)), discarding any sources outside the IRAC FOV. Due to the low count rate for GALEX, its background is best treated as a Poissonian distribution whereas the background for Spitzer IRAC images can be treated as Gaussian. Because of this difference, we employ the IRAF routine 'phot' for photometry and background estimation. The flux in each band is measured in circular apertures for each association, subtracting the local background through iterative rejection using the median value for GALEX and mode for Spitzer images. For efficiency's sake, a single aperture size is chosen for each galaxy (see Table 3) and applied to all associations within that galaxy. Since all of our associations are point sources at the resolution of GALEX and IRAC, we can then apply an appropriate aperture correction.

All apertures are equal to or greater than 5 pixels (7.5"), so no aperture correction is

Table 2. Image Information

\begin{tabular}{|c|c|c|c|c|c|c|c|c|}
\hline \multirow{2}{*}{$\begin{array}{l}\text { Target } \\
\text { Field }\end{array}$} & \multirow{2}{*}{$\begin{array}{c}\text { RA } \\
(\mathrm{J} 2000)\end{array}$} & \multirow{2}{*}{$\begin{array}{c}\text { Dec } \\
(\mathrm{J} 2000)\end{array}$} & \multirow{2}{*}{$\begin{array}{c}\text { Galactocentric } \\
\text { Distance }\end{array}$} & \multicolumn{3}{|c|}{ Exposure Time (s) } & \multicolumn{2}{|c|}{$3 \sigma$ Sensitivity $^{\mathrm{a}}$} \\
\hline & & & & FUV & NUV & IRAC & FUV & $3.6 \mu \mathrm{m}$ \\
\hline NGC 0628-OUT & 013702.00 & +155505.00 & $9.5^{\prime}\left(1.8 R_{o p t}\right)$ & 1644.0 & 1644.0 & 1900.0 & $3.46 \times 10^{-18}$ & $1.02 \times 10^{-20}$ \\
\hline NGC 2090-OUT & 054709.50 & -341127.00 & $4.1^{\prime}\left(1.7 R_{o p t}\right)$ & 2281.0 & 4107.1 & 1800.0 & $3.95 \times 10^{-18}$ & $9.60 \times 10^{-21}$ \\
\hline NGC 2841-OUT & 092221.50 & +505033.00 & 9.3' $\left(2.3 R_{o p t}\right)$ & 1775.4 & 1775.5 & 1900.0 & $1.94 \times 10^{-18}$ & $7.01 \times 10^{-21}$ \\
\hline NGC 3621-OUT & 111759.42 & -323942.60 & $10.1^{\prime}\left(1.7 R_{o p t}\right)$ & 7269.1 & 7269.1 & 1800.0 & $2.00 \times 10^{-18}$ & $1.00 \times 10^{-20}$ \\
\hline NGC 5055-OUT & 131621.70 & +415142.00 & $12.9^{\prime}\left(2.1 R_{o p t}\right)$ & 1660.0 & 1660.0 & 1900.0 & $1.76 \times 10^{-18}$ & $8.09 \times 10^{-21}$ \\
\hline
\end{tabular}

a Sensitivities are given in units of $\operatorname{erg~s}^{-1} \mathrm{~cm}^{-2} \stackrel{\circ}{A}^{-1}$ pixel $^{-1}$. The FUV sensitivities have been corrected for Galactic extinction (Table 3). 
needed for the GALEX fluxes (FUV FWHM 4.5"). To find the aperture correction for the IRAC bands, we referred to the IRAC Handbook and the point source aperture corrections found there. For our smallest apertures, this amounted to a $\sim 5 \%$ correction. Taking into account the crowding of the field, a sufficiently large annulus for each source was chosen to measure the local background. In some cases, the flux of the source in one or more bands was very faint and with background subtraction, these fluxes became negative. In these cases, a $3 \sigma$ upper limit is adopted. The fluxes measured in all six bands are used to derive each association's spectral energy distribution (SED). Since to determine the age/mass of an association we will need to fit a model to its SED, and since we have only a few datapoints per association (less than six, as will be explained in Section 4), those associations that are missing the NUV or $3.6 \mu \mathrm{m}$ point are discarded and those missing the $4.5 \mu \mathrm{m}$ point are flagged. Finally, the two GALEX bands are corrected for foreground Galactic extinction using the values in Table 3 .

Two main sources of noise dominate the uncertainty on each flux measurement: the noise from the source detection and the variance of the local background. The noise from the source for UV and MIR bands is the Poissonian noise of the measurement, converted to electrons. The background noise is the standard deviation on the sky pixels distribution, also converted into electrons. This is then multiplied by the square root of the number of pixels in the aperture. The two contributions are added in quadrature. For IRAC data, the uncertainty is dominated by the variance in the background; for the UV bands, the dominant source of error is the uncertainty in the source counts.

The total number of sources is listed in Table 3 for each galaxy. Contaminating stars and galaxies are then eliminated with a number of methods. From a visual inspection, foreground stars are removed by discarding any source with diffraction spikes in the original $3.6 \mu \mathrm{m}$ image. Similarly, any source with visible extended emission at $3.6 \mu \mathrm{m}$ is rejected.

Table 3. Photometry

\begin{tabular}{ccccc}
\hline \hline Galaxy & $\begin{array}{c}\text { Aperture } \\
\text { Diameter (arcseconds) }\end{array}$ & $\begin{array}{c}\text { Foreground Galactic } \\
\text { Extinction } E(B-V)^{\mathrm{a}}\end{array}$ & $\begin{array}{c}\text { Total } \\
\text { \# Sources }\end{array}$ & $\begin{array}{c}\text { Final } \\
\text { \# Sources }\end{array}$ \\
\hline NGC 0628 & 7.5 & 0.07 & 48 & 32 \\
NGC 2090 & 7.5 & 0.04 & 63 & 47 \\
NGC 2841 & 10.5 & 0.016 & 87 & 75 \\
NGC 3621 & 7.5 & 0.08 & 67 & 58 \\
NGC 5055 & 12.0 & 0.018 & 146 & 17 \\
\hline
\end{tabular}

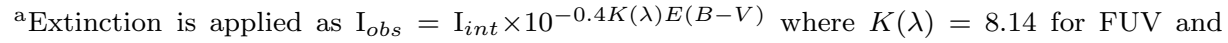
8.74 for NUV (Wyder et al. 2005). Extinction values for each galaxy are from the dust maps of Schlegel et al. (1998). 
Dim, low-mass stars are eliminated by imposing the criteria:

$$
\log \left(\frac{f_{\lambda}(F U V)}{f_{\lambda}(N U V)}\right)>-0.5
$$

which will eliminate sources that are red in the GALEX bands (lifetimes $\gtrsim 1$ Gyr). This criterion will also remove clusters with ages $\gtrsim 1$ Gyr, but this is acceptable as our primary goal is to identify clusters of recent star formation. 
Table 4. Source List

\begin{tabular}{|c|c|c|c|c|c|c|c|c|}
\hline $\begin{array}{c}\text { Association } \\
\text { ID }\end{array}$ & $\begin{array}{c}\text { RA }(J 2000) \\
\text { degree }\end{array}$ & $\begin{array}{c}\text { Dec }(J 2000) \\
\text { degree }\end{array}$ & $\begin{array}{l}\text { FUV }^{\mathrm{a}} \\
F_{\lambda}(1 \sigma)\end{array}$ & $\begin{array}{l}\mathrm{NUV}^{\mathrm{a}} \\
F_{\lambda}(1 \sigma)\end{array}$ & $\begin{array}{c}3.6^{\mathrm{a}} \\
F_{\lambda}(1 \sigma)\end{array}$ & $\begin{array}{c}4.5^{\mathrm{a}} \\
F_{\lambda}(1 \sigma)\end{array}$ & $\begin{array}{c}5.8^{\mathrm{a}} \\
F_{\lambda}(1 \sigma)\end{array}$ & $\begin{array}{c}8.0^{\mathrm{a}} \\
F_{\lambda}(1 \sigma)\end{array}$ \\
\hline \multicolumn{9}{|c|}{$\underline{\text { NGC } 0628 \text { Associations }}$} \\
\hline 1 & 013700.55 & +155118.9 & $2437.54(194.23)$ & $900.84(51.99)$ & $3.09(0.47)$ & $-0.58(0.94)$ & $-8.84(1.25)$ & $4.04(0.50)$ \\
\hline 2 & 013703.67 & +155429.4 & $919.19(119.96)$ & $240.34(32.49)$ & $4.14(0.17)$ & $2.09(0.17)$ & $-4.47(0.45)$ & $3.97(0.24)$ \\
\hline 3 & 013704.82 & +155427.9 & $814.75(113.36)$ & $296.35(32.88)$ & $14.79(0.18)$ & $8.58(0.16)$ & $-6.37(0.45)$ & $6.18(0.26)$ \\
\hline 4 & $0137 \quad 15.63$ & +155433.8 & $239.11(67.21)$ & $31.49(24.33)$ & $0.46(0.36)$ & $0.40(0.343)$ & $5.79(2.65)$ & $5.33(0.48)$ \\
\hline 5 & 013656.92 & +155411.5 & $283.53(73.81)$ & $230.79(31.92)$ & $0.42(0.18)$ & $0.30(0.19)$ & $6.88(1.53)$ & $-4.58(0.85)$ \\
\hline 6 & 013656.08 & +155356.3 & $457.59(93.03)$ & $152.05(32.41)$ & $2.71(0.18)$ & $1.12(0.21)$ & $-7.75(0.47)$ & $-1.173(0.88)$ \\
\hline 7 & 013656.81 & +155354.9 & $536.67(98.15)$ & $264.49(37.79)$ & $8.69(0.19)$ & $3.50(0.19)$ & $-2.26(0.46)$ & $1.88(0.28)$ \\
\hline 8 & 013657.64 & +155348.9 & $619.57(104.46)$ & $379.81(38.67)$ & $2.60(0.18)$ & $0.52(0.18)$ & $1.02(1.37)$ & $3.39(0.30)$ \\
\hline 9 & 013658.26 & +155339.7 & $851.90(119.06)$ & $380.60(37.32)$ & $5.56(0.20)$ & $3.00(0.18)$ & $-6.34(0.47)$ & $9.40(0.27)$ \\
\hline 10 & 013656.81 & +155335.3 & $158.49(67.90)$ & $13.24(29.49)$ & $0.90(0.20)$ & $0.21(0.20)$ & $2.34(1.37)$ & $-3.79(0.88)$ \\
\hline 11 & 013701.06 & +155327.9 & $1480.79(150.17)$ & $650.13(43.98)$ & $8.87(0.18)$ & $5.95(0.18)$ & $-16.98(0.47)$ & $4.64(0.25)$ \\
\hline 12 & 013702.84 & +155317.4 & $860.31(119.75)$ & $289.97(36.80)$ & $0.82(0.19)$ & $0.11(0.19)$ & $0.76(1.37)$ & $1.99(0.29)$ \\
\hline 13 & 013654.94 & +155257.9 & $4557.66(260.95)$ & $2000.80(70.88)$ & $21.66(0.28)$ & $12.07(0.26)$ & $-25.76(0.63)$ & $11.08(0.38)$ \\
\hline 14 & 013704.29 & +155303.9 & $1043.19(128.71)$ & $550.51(42.79)$ & $2.22(0.21)$ & $1.25(0.20)$ & $0.78(1.50)$ & $1.30(0.27)$ \\
\hline 15 & 013703.15 & +155320.4 & $948.72(124.94)$ & $322.47(38.13)$ & $0.44(0.18)$ & $-0.09(0.59)$ & $6.47(1.36)$ & $2.06(0.26)$ \\
\hline 16 & 013653.48 & +155250.4 & $847.97(128.13)$ & $440.40(47.94)$ & $16.13(0.40)$ & $7.61(0.37)$ & $-7.33(1.00)$ & $7.09(0.45)$ \\
\hline 17 & 013659.62 & +155244.4 & $559.76(97.36)$ & $335.42(36.21)$ & $0.40(0.20)$ & $0.07(0.21)$ & $-3.55(0.50)$ & $3.75(0.32)$ \\
\hline 18 & 013714.59 & +155241.3 & $816.66(115.29)$ & $594.56(42.92)$ & $29.29(0.43)$ & $11.82(0.38)$ & $-18.67(1.15)$ & $31.54(0.59)$ \\
\hline 19 & 013657.22 & +155223.4 & $913.49(121.48)$ & $383.49(37.48)$ & $0.44(0.21)$ & $-0.27(0.66)$ & $-6.84(0.55)$ & $-0.12(0.91)$ \\
\hline 20 & 013659.09 & +155214.4 & $594.98(102.33)$ & $401.09(38.27)$ & $5.90(0.21)$ & $1.74(0.20)$ & $-3.44(0.53)$ & $2.47(0.30)$ \\
\hline 21 & 013701.90 & +155154.9 & $521.07(97.57)$ & $110.31(28.12)$ & $6.56(0.27)$ & $4.21(0.26)$ & $-3.27(0.65)$ & $5.10(0.33)$ \\
\hline 22 & 013659.82 & +155141.4 & $574.64(102.96)$ & $76.85(33.09)$ & $4.67(0.28)$ & $1.43(0.23)$ & $-8.95(0.68)$ & $-0.25(1.08)$ \\
\hline 23 & 013659.41 & +155120.6 & $1624.18(161.02)$ & $587.02(47.46)$ & $1.45(0.41)$ & $0.59(0.33)$ & $4.42(2.77)$ & $4.33(0.44)$ \\
\hline 24 & 013708.88 & +155720.3 & $799.41(111.27)$ & $417.43(37.94)$ & $0.42(0.32)$ & $0.11(0.26)$ & $7.61(2.02)$ & $-5.70(1.15)$ \\
\hline 25 & 013700.14 & +155621.9 & $780.61(111.15)$ & $337.49(35.63)$ & $0.33(0.29)$ & $0.02(0.24)$ & $3.45(1.59)$ & $-2.84(0.95)$ \\
\hline 26 & 013701.18 & +155624.9 & $520.29(93.26)$ & $415.42(37.51)$ & $16.74(0.27)$ & $8.83(0.24)$ & $-12.25(0.50)$ & $9.60(0.32)$ \\
\hline 27 & 013701.60 & +155615.9 & $458.59(88.87)$ & $306.97(35.15)$ & $26.41(0.29)$ & $13.30(0.24)$ & $-22.01(0.49)$ & $21.52(0.35)$ \\
\hline 28 & 013651.09 & +155559.4 & $422.11(84.89)$ & $176.37(30.52)$ & $1.41(0.30)$ & $-1.52(1.08)$ & $4.23(2.53)$ & $-6.46(1.66)$ \\
\hline 29 & 013655.58 & +155556.6 & $254.87(70.40)$ & $10.95(24.27)$ & $0.43(0.22)$ & $0.25(0.191)$ & $3.48(1.44)$ & $-2.55(0.88)$ \\
\hline 30 & 013658.27 & +155559.3 & $356.63(79.26)$ & $223.52(32.48)$ & $2.03(0.29)$ & $1.43(0.22)$ & $-0.87(0.52)$ & $1.60(0.30)$ \\
\hline 31 & 013645.68 & +155508.4 & $1284.08(140.80)$ & $424.77(40.05)$ & $14.25(0.29)$ & $6.96(0.18)$ & $-32.59(0.95)$ & $13.12(0.39)$ \\
\hline 32 & $\begin{array}{lll}01 & 37 & 02.01\end{array}$ & +155445.9 & $1306.73(142.05)$ & $593.00(43.65)$ & $3.71(0.18)$ & $1.58(0.17)$ & $-4.32(0.44)$ & $-0.96(0.75)$ \\
\hline \multicolumn{9}{|c|}{$\underline{\text { NGC } 2090 \text { Associations }}$} \\
\hline 1 & 054655.00 & -34157.0 & $2969.17(189.08)$ & $1396.36(52.26)$ & $124.04(0.31)$ & $47.05(0.41)$ & $-105.98(0.67)$ & $57.91(0.51)$ \\
\hline 2 & 05476.86 & $-34 \quad 1537.0$ & $4684.45(236.49)$ & $2519.19(63.93)$ & $76.55(2.25)$ & $6.83(0.57)$ & $-21.60(1.84)$ & $4.60(0.66)$ \\
\hline 3 & $\begin{array}{lll}05 & 47 & 10.37\end{array}$ & $-34 \quad 15 \quad 38.5$ & $2130.59(185.67)$ & $1241.85(60.10)$ & $9.97(0.41)$ & $5.60(0.43)$ & $-21.39(1.17)$ & $7.38(0.43)$ \\
\hline 4 & 05478.67 & -341231.0 & $3947.28(241.72)$ & $1846.38(70.42)$ & $14.14(0.47)$ & $5.90(0.24)$ & $-55.52(0.57)$ & $61.23(0.49)$ \\
\hline 5 & $\begin{array}{lll}05 & 47 & 10.12\end{array}$ & $-34 \quad 12 \quad 16.0$ & $1224.98(137.42)$ & $472.97(40.79)$ & $2.85(0.25)$ & $2.37(0.19)$ & $5.39(1.45)$ & $-0.93(0.65)$ \\
\hline 6 & 05478.06 & $-34 \quad 1205.5$ & $10001.50(312.69)$ & $4853.41(73.08)$ & $20.15(0.36)$ & $9.30(0.22)$ & $-96.61(0.47)$ & $122.38(0.31)$ \\
\hline 7 & $\begin{array}{lll}05 & 47 & 12.78\end{array}$ & $-34 \quad 1229.5$ & $423.18(108.95)$ & $305.41(42.42)$ & $16.89(0.35)$ & $7.71(0.22)$ & $-6.68(0.53)$ & $5.69(0.27)$ \\
\hline 8 & 05473.35 & $-34 \quad 1253.5$ & $3197.48(212.51)$ & $1403.25(63.67)$ & $15.08(0.68)$ & $4.50(0.36)$ & $-45.47(0.59)$ & $54.68(0.39)$ \\
\hline 9 & $05 \quad 475.77$ & -341320.5 & $3837.07(223.56)$ & $1663.02(81.63)$ & $5.37(1.22)$ & $2.40(0.51)$ & $-28.60(0.82)$ & $35.60(0.61)$ \\
\hline 10 & 05476.01 & $-34 \quad 1308.5$ & $5148.20(269.62)$ & $2851.27(102.05)$ & $28.27(1.12)$ & $12.92(0.53)$ & $-47.25(0.85)$ & $49.99(0.64)$ \\
\hline 11 & $\begin{array}{lll}05 & 47 & 12.42\end{array}$ & $-34 \quad 1240.0$ & $1465.53(140.24)$ & $1047.07(44.16)$ & $65.52(0.38)$ & $26.55(0.21)$ & $-37.63(0.46)$ & $15.88(0.22)$ \\
\hline 12 & 05470.93 & $-34 \quad 12 \quad 44.5$ & $2381.87(182.38)$ & $1117.98(61.64)$ & $4.34(1.06)$ & $2.37(0.53)$ & $-27.82(0.68)$ & $35.78(0.49)$ \\
\hline 13 & 054659.96 & $-34 \quad 1314.5$ & $2466.75(210.55)$ & $1145.70(79.01)$ & $9.38(0.82)$ & $5.27(0.52)$ & $-22.62(0.71)$ & $26.94(0.60)$ \\
\hline 14 & $05 \quad 470.81$ & $-34 \quad 1326.5$ & $6007.34(308.05)$ & $2798.25(97.99)$ & $35.02(1.30)$ & $17.01(0.65)$ & $-163.75(1.17)$ & $201.18(0.96)$ \\
\hline 15 & 05472.38 & $-34 \quad 13 \quad 17.5$ & $1839.48(244.93)$ & $1133.02(100.58)$ & $16.09(0.69)$ & $7.14(0.62)$ & $-63.33(1.12)$ & $67.55(0.86)$ \\
\hline 16 & 054655.61 & $-34 \quad 1246.0$ & $951.98(121.36)$ & $748.09(38.71)$ & $31.43(0.39)$ & $21.13(0.28)$ & $-18.55(0.95)$ & $53.60(0.32)$ \\
\hline 17 & 054711.93 & $-34 \quad 1256.5$ & $2704.80(184.09)$ & $1124.76(48.06)$ & $4.22(0.48)$ & $1.11(0.27)$ & $-3.60(0.53)$ & $1.09(0.27)$ \\
\hline 18 & 05471.05 & $-34 \quad 1259.5$ & $528.65(158.75)$ & $52.64(56.23)$ & $7.45(0.48)$ & $4.18(0.33)$ & $-4.10(0.63)$ & $10.78(0.60)$ \\
\hline 19 & $05 \quad 477.70$ & $-34 \quad 13 \quad 32.4$ & $2534.68(185.20)$ & $1087.31(60.35)$ & $1.29(0.68)$ & $1.12(0.37)$ & $-6.98(0.65)$ & $20.39(0.44)$ \\
\hline 20 & 05478.06 & -341316.0 & $2560.97(203.73)$ & $1199.08(71.77)$ & $14.31(0.46)$ & $6.63(0.35)$ & $-18.78(0.55)$ & $14.28(0.38)$ \\
\hline 21 & $05 \quad 478.43$ & -341323.5 & $3071.10(216.29)$ & $1472.02(69.46)$ & $15.57(0.57)$ & $7.74(0.40)$ & $-16.94(0.63)$ & $13.70(0.37)$ \\
\hline 22 & $05 \quad 4657.42$ & -341325.0 & $8094.95(281.58)$ & $3917.28(72.04)$ & $20.00(0.67)$ & $8.40(0.40)$ & $-25.97(1.08)$ & $18.05(0.44)$ \\
\hline 23 & 054656.82 & -341331.0 & $4349.20(215.44)$ & $1744.69(58.60)$ & $25.49(0.78)$ & $11.36(0.40)$ & $-38.83(1.19)$ & $46.17(0.45)$ \\
\hline 24 & $05 \quad 47 \quad 10.60$ & $-34 \quad 1319.0$ & $448.14(128.14)$ & $207.90(45.75)$ & $4.00(0.52)$ & $3.08(0.34)$ & $0.14(1.69)$ & $-4.11(1.15)$ \\
\hline 25 & $0547 \quad 10.00$ & $-34 \quad 13 \quad 32.5$ & $1117.50(151.22)$ & $348.36(48.50)$ & $5.74(0.41)$ & $4.61(0.29)$ & $-5.67(0.58)$ & $4.84(0.36)$ \\
\hline 26 & 054654.04 & $-34 \quad 1341.5$ & $1955.90(145.86)$ & $634.46(37.87)$ & $5.21(0.50)$ & $2.61(0.33)$ & $-18.63(1.17)$ & $1.41(0.46)$ \\
\hline 27 & 05478.67 & $-34 \quad 1359.5$ & $1385.48(167.75)$ & $754.54(50.53)$ & $19.31(0.40)$ & $8.45(0.28)$ & $-12.24(0.61)$ & $27.71(0.47)$ \\
\hline
\end{tabular}


Table 4-Continued

\begin{tabular}{|c|c|c|c|c|c|c|c|c|}
\hline $\begin{array}{c}\text { Association } \\
\text { ID }\end{array}$ & $\begin{array}{c}\text { RA }(\mathrm{J} 2000) \\
\text { degree }\end{array}$ & $\begin{array}{c}\text { Dec(J2000) } \\
\text { degree }\end{array}$ & $\begin{array}{l}\mathrm{FUV}^{\mathrm{a}} \\
F_{\lambda}(1 \sigma)\end{array}$ & $\begin{array}{l}\mathrm{NUV}^{\mathrm{a}} \\
F_{\lambda}(1 \sigma)\end{array}$ & $\begin{array}{c}3.6^{\mathrm{a}} \\
F_{\lambda}(1 \sigma) \\
\end{array}$ & $\begin{array}{c}4.5^{\mathrm{a}} \\
F_{\lambda}(1 \sigma) \\
\end{array}$ & $\begin{array}{c}5.8^{\mathrm{a}} \\
F_{\lambda}(1 \sigma) \\
\end{array}$ & $\begin{array}{c}8.0^{\mathrm{a}} \\
F_{\lambda}(1 \sigma) \\
\end{array}$ \\
\hline 28 & $05 \quad 47 \quad 7.94$ & -341356.5 & $1827.04(172.14)$ & $839.27(58.78)$ & $12.50(0.48)$ & $5.69(0.34)$ & $-27.53(0.59)$ & $30.13(0.42)$ \\
\hline 30 & $0547 \quad 7.46$ & -341037.0 & $1216.87(152.99)$ & $714.25(41.47)$ & $3.67(0.25)$ & $1.27(0.18)$ & $-5.32(0.39)$ & $4.45(0.22)$ \\
\hline 31 & 054656.45 & -341455.0 & $3365.79(223.42)$ & $1248.85(72.55)$ & $15.75(1.30)$ & $6.96(0.56)$ & $-17.99(2.04)$ & $14.82(0.51)$ \\
\hline 32 & 05476.61 & -341035.5 & $1611.63(139.48)$ & $712.82(39.86)$ & $4.54(0.26)$ & $3.36(0.17)$ & $-9.08(0.44)$ & $2.18(0.22)$ \\
\hline 33 & 05479.15 & -341444.5 & $2945.92(191.89)$ & $1567.68(56.27)$ & $2.55(0.77)$ & $2.67(0.34)$ & $-3.06(0.71)$ & $2.88(0.51)$ \\
\hline 35 & $0547 \quad 11.09$ & $\begin{array}{lll}-34 & 15 & 04.0\end{array}$ & $2222.31(174.96)$ & $1235.16(46.36)$ & $0.67(0.40)$ & $-0.46(1.03)$ & $10.61(2.56)$ & $5.37(0.41)$ \\
\hline 36 & $\begin{array}{lll}05 & 47 & 10.12\end{array}$ & $-34 \quad 1041.5$ & $725.99(135.27)$ & $353.50(35.30)$ & $6.04(0.29)$ & $2.23(0.21)$ & $-8.46(0.42)$ & $6.77(0.19)$ \\
\hline 37 & 05479.88 & -341520.5 & $1060.87(135.71)$ & $112.43(44.66)$ & $0.76(0.40)$ & $0.42(0.35)$ & $10.00(2.61)$ & $1.66(0.40)$ \\
\hline 38 & 05479.63 & $-3410 \quad 47.5$ & $492.59(113.03)$ & $141.07(36.93)$ & $1.72(0.26)$ & $1.19(0.19)$ & $2.46(1.25)$ & $-0.08(0.67)$ \\
\hline 39 & 054655.61 & -341056.5 & $1764.14(142.19)$ & $797.93(34.31)$ & $48.77(0.25)$ & $32.4(0.23)$ & $-63.81(0.74)$ & $44.75(0.31)$ \\
\hline 40 & $05 \quad 477.34$ & -341114.5 & $1765.56(153.99)$ & $748.66(38.94)$ & $24.68(0.35)$ & $9.31(0.21)$ & $-21.91(0.52)$ & $15.21(0.27)$ \\
\hline 41 & 05477.58 & -341135.5 & $1693.53(171.97)$ & $820.86(49.51)$ & $5.37(0.32)$ & $2.81(0.20)$ & $-16.23(0.41)$ & $20.28(0.21)$ \\
\hline 42 & $\begin{array}{lll}05 & 47 & 10.24\end{array}$ & -341117.5 & $2248.85(166.58)$ & $1038.72(47.25)$ & $13.98(0.25)$ & $6.45(0.19)$ & $-8.99(0.49)$ & $7.43(0.25)$ \\
\hline 43 & 054710.60 & -341138.5 & $2209.60(201.46)$ & $766.97(56.76)$ & $26.97(0.48)$ & $14.41(0.25)$ & $-27.71(0.49)$ & $20.74(0.28)$ \\
\hline 44 & $\begin{array}{lll}05 & 47 & 12.17\end{array}$ & -341143.0 & $11845.50(337.68)$ & $5032.86(90.28)$ & $28.38(0.67)$ & $13.52(0.36)$ & $-51.88(0.58)$ & $58.93(0.38)$ \\
\hline 46 & 05474.32 & -341210.0 & $3699.32(231.19)$ & $1873.34(67.15)$ & $15.54(0.64)$ & $6.79(0.32)$ & $-20.52(0.53)$ & $26.15(0.31)$ \\
\hline 47 & $05 \quad 476.25$ & -341225.0 & $3968.03(234.56)$ & $2137.33(67.25)$ & $6.44(0.63)$ & $3.83(0.26)$ & $-25.62(0.49)$ & $32.25(0.38)$ \\
\hline \multicolumn{9}{|c|}{$\underline{\text { NGC } 2841 \text { Associations }}$} \\
\hline 1 & $\begin{array}{lll}09 & 22 & 16.22\end{array}$ & +505409.9 & $1320.07(123.91)$ & 193.05(35.09) & $1.29(0.60)$ & $-0.09(1.32)$ & $-4.18(1.04)$ & $5.25(0.70)$ \\
\hline 2 & $\begin{array}{lll}09 & 22 & 17.81\end{array}$ & +505355.0 & $334.37(97.45)$ & $345.14(38.81)$ & $7.83(0.63)$ & $1.77(0.40)$ & $-6.74(1.13)$ & $10.31(0.69)$ \\
\hline 3 & $0922 \quad 21.78$ & +505346.3 & $221.43(84.78)$ & $212.41(35.67)$ & 19.94(0.49) & $8.50(0.38)$ & $-19.82(0.93)$ & $11.25(0.66)$ \\
\hline 4 & $\begin{array}{lll}09 & 22 & 22.09\end{array}$ & +505358.3 & $1578.65(132.69)$ & $112.54(34.29)$ & $6.67(0.55)$ & $5.61(0.45)$ & $-31.07(0.86)$ & $8.39(0.69)$ \\
\hline 5 & $\begin{array}{lll}09 & 22 & 19.24\end{array}$ & +505353.6 & $2009.15(148.87)$ & 691.77(43.6) & $9.44(0.55)$ & $3.38(0.39)$ & $-8.59(1.01)$ & $16.79(0.64)$ \\
\hline 6 & $0922 \quad 20.36$ & +505343.2 & 1469.41(139.70) & $744.21(48.19)$ & $4.49(0.55)$ & $0.62(0.41)$ & $5.02(2.78)$ & $2.00(0.63)$ \\
\hline 7 & 092230.20 & +505319.7 & $2029.19(156.30)$ & $1031.96(51.49)$ & $14.15(0.44)$ & $4.79(0.27)$ & $-11.25(1.19)$ & $11.89(0.58)$ \\
\hline 8 & 092226.22 & +505339.0 & $2388.51(157.14)$ & $479.12(41.8)$ & $5.27(0.43)$ & $2.12(0.46)$ & $0.82(2.79)$ & $-0.36(1.86)$ \\
\hline 9 & $0922 \quad 27.34$ & +505327.1 & $1993.82(154.72)$ & $530.70(41.98)$ & $10.34(0.50)$ & $7.19(0.37)$ & $-16.07(0.99)$ & $10.49(0.50)$ \\
\hline 10 & 092205.28 & +505357.2 & $1053.60(125.19)$ & $131.60(31.68)$ & $1.41(0.83)$ & $-0.18(2.19)$ & $0.13(6.7)$ & $2.43(1.13)$ \\
\hline 11 & 092205.79 & +505318.2 & $1120.28(127.49)$ & $256.47(33.66)$ & $15.14(0.78)$ & $7.72(0.48)$ & $-53.38(1.65)$ & $2.39(0.75)$ \\
\hline 12 & 092208.65 & +505301.9 & $1345.86(135.01)$ & $94.57(34.93)$ & $7.85(0.45)$ & $4.0(0.32)$ & $-3.39(1.05)$ & $-2.56(1.53)$ \\
\hline 13 & $\begin{array}{lll}09 & 22 & 19.11\end{array}$ & +505310.1 & $1030.51(124.38)$ & $157.87(32.91)$ & $8.43(0.39)$ & $6.32(0.32)$ & $-19.52(0.86)$ & $-3.34(1.35)$ \\
\hline 14 & 092217.85 & +505258.0 & $1183.14(129.63)$ & $205.43(34.44)$ & $12.23(0.29)$ & $5.37(0.25)$ & $-15.34(0.85)$ & $4.37(0.36)$ \\
\hline 18 & 092207.40 & +505242.3 & $1354.96(125.78)$ & $226.26(34.88)$ & $5.28(0.41)$ & $2.89(0.30)$ & $9.26(3.65)$ & $3.15(0.46)$ \\
\hline 19 & 092213.30 & +505147.2 & $1446.57(128.43)$ & $195.54(34.02)$ & $8.27(0.30)$ & $4.49(0.28)$ & $2.36(1.66)$ & $-0.27(1.16)$ \\
\hline 20 & 092210.10 & +505238.0 & $1840.59(141.29)$ & $726.43(45.87)$ & $40.87(0.31)$ & $23.56(0.28)$ & $-35.19(0.80)$ & $31.56(0.39)$ \\
\hline 21 & $0922 \quad 10.74$ & +505224.6 & $2010.29(146.08)$ & $338.14(37.49)$ & $2.01(0.36)$ & $1.51(0.26)$ & $7.97(2.13)$ & $-0.93(1.25)$ \\
\hline 22 & $0922 \quad 11.70$ & +505214.1 & $2105.25(149.41)$ & $688.25(43.3)$ & $3.21(0.27)$ & $0.69(0.26)$ & $-3.93(0.68)$ & $2.10(0.39)$ \\
\hline 23 & 092205.35 & +505230.2 & $1126.54(127.71)$ & $175.95(33.21)$ & $21.08(0.48)$ & $3.21(0.39)$ & $-1.82(1.57)$ & $9.08(0.52)$ \\
\hline 24 & $\begin{array}{lll}09 & 22 & 14.54\end{array}$ & +505226.3 & $1253.70(131.99)$ & $226.47(34.92)$ & $0.40(0.24)$ & $-0.52(0.77)$ & $-3.26(0.67)$ & $0.11(0.34)$ \\
\hline 25 & $\begin{array}{lll}09 & 22 & 04.87\end{array}$ & +505234.7 & $1046.15(124.93)$ & $193.89(33.44)$ & $26.14(0.48)$ & $5.49(0.39)$ & $25.28(5.23)$ & $1.7(0.59)$ \\
\hline 26 & $09 \quad 2232.62$ & +505210.9 & $1220.23(130.88)$ & $155.50(32.43)$ & $13.36(0.25)$ & $4.88(0.25)$ & $-13.36(0.78)$ & $3.92(0.36)$ \\
\hline 27 & $0922 \quad 28.98$ & +505204.7 & $1174.87(129.35)$ & $279.07(33.91)$ & $13.65(0.22)$ & $13.03(0.21)$ & $-31.81(0.63)$ & $18.22(0.32)$ \\
\hline 28 & $09 \quad 2207.91$ & +505151.4 & $1069.05(125.72)$ & $489.03(38.00)$ & $7.50(0.27)$ & $4.78(0.30)$ & $-3.86(0.65)$ & $9.77(0.46)$ \\
\hline 29 & $0922 \quad 12.82$ & +505202.2 & $1955.41(153.49)$ & $344.88(38.27)$ & $1.67(0.22)$ & $0.76(0.23)$ & $2.64(1.78)$ & $-1.05(1.15)$ \\
\hline 30 & 092218.07 & +505132.5 & $1549.77(131.87)$ & $259.02(39.38)$ & $1.53(0.25)$ & $-0.46(0.68)$ & $-0.64(0.57)$ & $-0.09(0.95)$ \\
\hline 31 & $\begin{array}{lll}09 & 22 & 19.50\end{array}$ & +505122.1 & $2212.00(139.09)$ & $768.34(41.33)$ & $5.60(0.29)$ & $2.81(0.19)$ & $-11.36(0.52)$ & $7.27(0.30)$ \\
\hline 32 & 092226.75 & +505224.0 & $846.40(117.73)$ & $35.11(32.09)$ & $2.88(0.22)$ & $2.65(0.22)$ & $-2.87(0.59)$ & $-4.57(1.01)$ \\
\hline 33 & 092232.16 & +505142.3 & $1138.30(128.11)$ & $47.33(29.52)$ & $16.75(0.20)$ & $10.81(0.21)$ & $-20.09(0.56)$ & $6.64(0.31)$ \\
\hline 34 & 092237.08 & +505130.6 & $834.16(117.27)$ & $379.65(37.28)$ & $9.09(0.23)$ & $5.66(0.25)$ & $-5.7(0.62)$ & $6.68(0.41)$ \\
\hline 35 & 092224.72 & +505128.4 & $1073.20(125.87)$ & $26.12(30.22)$ & $3.88(0.24)$ & $2.74(0.25)$ & $-8.88(0.50)$ & $0.84(0.34)$ \\
\hline 36 & 092229.16 & +505122.7 & $748.47(114.03)$ & $18.09(28.45)$ & $11.04(0.20)$ & $8.47(0.19)$ & $-20.46(0.55)$ & $14.42(0.30)$ \\
\hline 37 & 092235.66 & +505115.5 & $1200.05(130.20)$ & $223.37(37.2)$ & $9.22(0.23)$ & $1.95(0.22)$ & $2.36(1.66)$ & $-0.24(1.09)$ \\
\hline 38 & 092235.03 & +505117.0 & $1453.31(138.45)$ & $335.09(36.25)$ & $2.43(0.23)$ & $0.57(0.21)$ & $0.33(1.55)$ & $4.87(0.34)$ \\
\hline 39 & 092227.43 & +505113.6 & $997.48(123.21)$ & $124.17(32.61)$ & $4.79(0.22)$ & $2.20(0.19)$ & $-4.46(0.55)$ & $4.22(0.31)$ \\
\hline 40 & 092211.91 & +505106.6 & $769.79(114.85)$ & $134.24(32.25)$ & $3.96(0.25)$ & $1.61(0.21)$ & $-3.13(0.61)$ & $2.91(0.36)$ \\
\hline 41 & 092215.88 & +505053.4 & $1313.19(133.95)$ & $396.04(37.16)$ & $40.78(0.24)$ & $23.90(0.23)$ & $-31.75(0.62)$ & $43.51(0.29)$ \\
\hline 42 & $0922 \quad 27.92$ & +505045.1 & $1263.25(132.31)$ & $537.98(39.09)$ & $40.57(0.22)$ & $24.01(0.19)$ & $-33.97(0.58)$ & $46.41(0.27)$ \\
\hline 43 & $\begin{array}{lll}09 & 22 & 18.26\end{array}$ & +505044.5 & $1359.08(135.44)$ & $301.91(36.04)$ & $48.96(0.26)$ & $24.45(0.19)$ & $-34.38(0.60)$ & $21.76(0.28)$ \\
\hline
\end{tabular}


Table 4-Continued

\begin{tabular}{|c|c|c|c|c|c|c|c|c|}
\hline $\begin{array}{c}\text { Association } \\
\text { ID }\end{array}$ & $\begin{array}{c}\text { RA }(\mathrm{J} 2000) \\
\text { degree }\end{array}$ & $\begin{array}{c}\operatorname{Dec}(\mathrm{J} 2000) \\
\text { degree }\end{array}$ & $\begin{array}{l}\mathrm{FUV}^{\mathrm{a}} \\
F_{\lambda}(1 \sigma)\end{array}$ & $\begin{array}{l}\mathrm{NUV}^{\mathrm{a}} \\
F_{\lambda}(1 \sigma)\end{array}$ & $\begin{array}{c}3.6^{\mathrm{a}} \\
F_{\lambda}(1 \sigma)\end{array}$ & $\begin{array}{c}4.5^{\mathrm{a}} \\
F_{\lambda}(1 \sigma)\end{array}$ & $\begin{array}{c}5.8^{\mathrm{a}} \\
F_{\lambda}(1 \sigma)\end{array}$ & $\begin{array}{c}8.0^{\mathrm{a}} \\
F_{\lambda}(1 \sigma)\end{array}$ \\
\hline 44 & 092223.01 & +505043.3 & $1125.50(127.67)$ & $176.24(34.30)$ & $9.23(0.23)$ & $5.48(0.20)$ & $-14.29(0.62)$ & $2.88(0.28)$ \\
\hline 46 & 092218.90 & +505035.6 & $1270.26(122.63)$ & $120.62(36.27)$ & $31.43(0.30)$ & $13.94(0.21)$ & $-24.55(0.66)$ & $2.42(0.29)$ \\
\hline 47 & $09 \quad 22 \quad 14.15$ & +505035.3 & $1201.74(130.26)$ & $146.14(32.07)$ & $1.13(0.21)$ & $0.05(0.23)$ & $-5.59(0.59)$ & $-1.01(1.04)$ \\
\hline 48 & $\begin{array}{lll}09 & 22 & 04.81\end{array}$ & +505022.7 & $923.86(120.57)$ & $316.96(36.32)$ & $56.11(0.26)$ & $37.01(0.30)$ & $-60.5(0.83)$ & $77.82(0.39)$ \\
\hline 49 & 092222.58 & +504932.8 & $2162.67(159.28)$ & $876.20(43.08)$ & $51.6(0.31)$ & $21.54(0.26)$ & $-28.35(0.51)$ & $10.23(0.34)$ \\
\hline 50 & 092221.13 & +505019.2 & $2789.44(168.50)$ & $770.36(43.86)$ & $1.66(0.22)$ & $0.52(0.21)$ & $-0.22(0.55)$ & $2.35(0.32)$ \\
\hline 51 & $0922 \quad 20.82$ & +505005.7 & $1930.70(154.92)$ & $636.17(49.62)$ & $5.17(0.27)$ & $2.34(0.21)$ & $-2.33(0.62)$ & $3.56(0.31)$ \\
\hline 52 & $09 \quad 22 \quad 44.72$ & +505020.5 & $997.70(123.22)$ & $112.78(31.58)$ & $13.96(0.55)$ & $6.74(0.47)$ & $-29.22(1.93)$ & $5.77(0.78)$ \\
\hline 53 & 092229.20 & +505016.7 & $1286.06(133.06)$ & $292.58(34.48)$ & $6.45(0.21)$ & $3.08(0.22)$ & $-8.27(0.59)$ & $8.24(0.33)$ \\
\hline 54 & 092209.58 & +505006.5 & $1313.43(133.95)$ & $520.04(38.53)$ & $8.67(0.20)$ & $4.36(0.19)$ & $-1.92(0.54)$ & $8.63(0.31)$ \\
\hline 55 & 092213.86 & +504951.8 & $1638.72(144.18)$ & $767.39(42.92)$ & $28.26(0.20)$ & $14.88(0.21)$ & $-14.35(0.50)$ & $10.25(0.35)$ \\
\hline 56 & $\begin{array}{lll}09 & 22 & 03.41\end{array}$ & +504957.1 & $809.94(116.36)$ & $128.64(31.26)$ & $13.92(0.28)$ & $6.27(0.31)$ & $-4.05(0.80)$ & $1.50(0.47)$ \\
\hline 57 & $\begin{array}{lll}09 & 22 & 33.17\end{array}$ & +504958.9 & $1044.35(124.86)$ & $89.98(32.39)$ & $5.07(0.32)$ & $2.30(0.22)$ & $5.47(1.75)$ & $0.84(0.36)$ \\
\hline 58 & 092227.00 & +504949.6 & $1373.71(135.91)$ & $419.63(36.40)$ & $10.73(0.23)$ & $5.19(0.27)$ & $-7.60(0.44)$ & $8.20(0.36)$ \\
\hline 59 & $0922 \quad 17.98$ & +504943.0 & $1120.40(127.50)$ & $158.65(31.19)$ & $9.36(0.23)$ & $4.38(0.21)$ & $-16.33(0.57)$ & $2.50(0.31)$ \\
\hline 60 & $0922 \quad 30.34$ & +504927.2 & $1380.51(136.13)$ & $263.74(33.90)$ & $4.38(0.20)$ & $2.31(0.19)$ & $1.47(1.66)$ & $-0.81(1.04)$ \\
\hline 62 & $0922 \quad 18.63$ & +504928.1 & $1816.12(149.47)$ & $435.71(34.49)$ & $7.37(0.20)$ & $3.54(0.20)$ & $-4.33(0.54)$ & $7.88(0.28)$ \\
\hline 63 & $\begin{array}{lll}09 & 22 & 19.11\end{array}$ & +504916.1 & $1449.66(138.33)$ & $466.22(37.73)$ & $6.94(0.21)$ & $3.16(0.17)$ & $-5.35(0.54)$ & $7.00(0.28)$ \\
\hline 64 & 092235.88 & +504929.0 & $714.19(112.71)$ & $299.91(33.71)$ & $4.23(0.28)$ & $3.37(0.24)$ & $3.55(1.99)$ & $0.01(0.42)$ \\
\hline 65 & 092230.53 & +504848.3 & $968.21(122.17)$ & $62.40(30.33)$ & $24.48(0.29)$ & $9.74(0.24)$ & $-11.21(0.62)$ & $7.76(0.33)$ \\
\hline 66 & 092229.43 & +504834.7 & $1122.03(127.55)$ & $85.10(31.04)$ & $0.57(0.26)$ & $0.02(0.24)$ & $-2.43(0.62)$ & $-1.39(1.11)$ \\
\hline 67 & $09 \quad 22 \quad 16.61$ & +504832.4 & $842.08(117.57)$ & $275.70(33.39)$ & $22.8(0.26)$ & $13.58(0.26)$ & $-16.25(0.64)$ & $12.85(0.37)$ \\
\hline 68 & $09 \quad 22 \quad 15.35$ & +504820.4 & $1458.53(138.61)$ & $436.95(35.66)$ & $25.33(0.36)$ & $11.02(0.36)$ & $-10.77(0.65)$ & $32.79(0.50)$ \\
\hline 69 & 092236.24 & +504823.1 & $1120.65(127.51)$ & $181.69(31.43)$ & $0.79(0.31)$ & $0.32(0.34)$ & $-17.03(1.14)$ & $3.79(0.48)$ \\
\hline 70 & 092208.56 & +504757.4 & $1065.19(125.59)$ & $40.48(29.72)$ & $1.73(0.40)$ & $1.61(0.33)$ & $-20.86(0.94)$ & $-1.6(1.57)$ \\
\hline 71 & 092202.09 & +504746.5 & $778.30(115.17)$ & $116.23(31.13)$ & $7.84(0.75)$ & $4.42(0.53)$ & $-1644.28(1.85)$ & $8.22(0.71)$ \\
\hline 72 & 092218.86 & +504737.1 & $886.54(119.21)$ & $108.67(32.21)$ & $56.23(0.35)$ & $29.05(0.28)$ & $-58.04(0.85)$ & $58.45(0.43)$ \\
\hline 73 & $0922 \quad 37.38$ & +504729.1 & $1017.91(123.93)$ & $47.87(29.78)$ & $71.27(0.56)$ & $12.01(0.39)$ & $43.17(4.94)$ & $9.42(0.63)$ \\
\hline 74 & 092204.64 & +504719.7 & $1010.94(123.69)$ & $476.48(37.56)$ & $8.05(0.45)$ & $3.41(0.29)$ & $-40.32(2.15)$ & $12.85(0.55)$ \\
\hline 75 & 092232.79 & +504725.9 & $921.70(120.49)$ & $200.82(32.45)$ & $60.05(0.46)$ & $35.17(0.35)$ & $-49.61(1.04)$ & $19.85(0.55)$ \\
\hline \multicolumn{9}{|c|}{ NGC 3621 Associations } \\
\hline 1 & 111757.78 & $\begin{array}{lll}-32 & 4306.8\end{array}$ & $2243.43(136.95)$ & $1260.11(45.39)$ & $0.12(0.37)$ & $0.12(0.30)$ & 8.06(1.91) & $-1.48(1.07)$ \\
\hline 2 & $\begin{array}{lll}11 & 17 & 59.20\end{array}$ & $\begin{array}{lll}-32 & 43 & 08.4\end{array}$ & $1998.36(138.01)$ & $1107.82(56.15)$ & $7.90(0.37)$ & $3.56(0.30)$ & $-18.34(0.56)$ & $22.62(0.30)$ \\
\hline 6 & 111801.70 & $-32 \quad 44 \quad 14.4$ & $9896.20(206.19)$ & $5294.60(63.85)$ & $15.99(0.91)$ & $9.58(0.77)$ & $-25.08(1.79)$ & $22.71(1.03)$ \\
\hline 7 & $\begin{array}{lll}11 & 18 & 04.43\end{array}$ & $\begin{array}{lll}-32 & 44 & 02.4\end{array}$ & $2855.58(132.46)$ & $1464.53(46.12)$ & $58.83(0.51)$ & $26.05(0.30)$ & $-37.75(1.30)$ & $22.59(0.67)$ \\
\hline 8 & $\begin{array}{lll}11 & 17 & 55.77\end{array}$ & -323950.3 & $587.16(77.47)$ & $368.14(31.61)$ & $6.92(0.28)$ & $2.82(0.21)$ & $-2.16(0.40)$ & $2.42(0.23)$ \\
\hline 9 & $\begin{array}{lll}11 & 18 & 10.02\end{array}$ & -324021.9 & $1301.39(103.12)$ & $707.34(38.63)$ & $23.19(0.25)$ & $9.35(0.19)$ & $-14.63(0.46)$ & $4.78(0.28)$ \\
\hline 10 & $\begin{array}{lll}11 & 18 & 13.71\end{array}$ & $\begin{array}{lll}-32 & 40 & 02.4\end{array}$ & $5242.53(172.64)$ & $2644.11(61.88)$ & $12.34(0.33)$ & $3.99(0.23)$ & $-13.33(0.57)$ & $14.81(0.35)$ \\
\hline 11 & $1118 \quad 12.28$ & -324006.9 & $2786.07(143.47)$ & $1422.47(55.31)$ & $3.21(0.30)$ & $1.09(0.21)$ & $2.20(1.56)$ & $6.07(0.33)$ \\
\hline 12 & 111803.85 & -323954.9 & 1359.93(97.29) & $682.65(31.06)$ & $33.70(0.27)$ & $12.70(0.21)$ & $-13.86(0.40)$ & $3.79(0.25)$ \\
\hline 13 & 111756.60 & $\begin{array}{lll}-32 & 40 & 03.8\end{array}$ & $1893.15(104.55)$ & $713.00(35.81)$ & $13.94(0.30)$ & $5.40(0.22)$ & $-8.15(0.37)$ & $6.51(0.23)$ \\
\hline 14 & $1118 \quad 15.73$ & -323954.9 & $949.06(83.63)$ & $434.29(27.91)$ & $1.53(0.32)$ & $-0.69(0.84)$ & $8.40(2.41)$ & $0.80(0.41)$ \\
\hline 15 & 111808.00 & $-32 \quad 4015.9$ & $6369.25(166.24)$ & $3195.22(48.58)$ & $7.45(0.23)$ & $3.64(0.17)$ & $-17.52(0.39)$ & $9.58(0.28)$ \\
\hline 16 & $11 \quad 1803.72$ & -324129.4 & $1566.48(112.34)$ & $849.89(42.95)$ & $1.84(0.23)$ & $0.13(0.18)$ & $-3.62(0.38)$ & $-1.48(0.79)$ \\
\hline 17 & $11 \quad 1806.34$ & $-32 \quad 4032.4$ & $1311.11(105.24)$ & $919.76(41.95)$ & $84.78(0.35)$ & $34.11(0.19)$ & $-33.9(0.44)$ & $11.38(0.26)$ \\
\hline 18 & $\begin{array}{lll}11 & 18 & 05.51\end{array}$ & $-32 \quad 4056.4$ & $8647.39(199.52)$ & $4877.93(66.34)$ & $15.98(0.32)$ & $7.36(0.18)$ & $-15.83(0.42)$ & $14.57(0.24)$ \\
\hline 19 & 111742.70 & -324030.6 & $355.50(64.19)$ & $239.26(26.55)$ & $20.23(0.34)$ & $10.06(0.33)$ & $-30.84(0.77)$ & $7.41(0.40)$ \\
\hline 20 & $11 \quad 1809.43$ & $-32 \quad 4041.4$ & $593.62(99.77)$ & $328.20(35.49)$ & $15.49(0.22)$ & $8.66(0.20)$ & $-11.41(0.46)$ & $5.01(0.28)$ \\
\hline 21 & $11 \quad 18 \quad 17.63$ & $\begin{array}{lll}-32 & 40 & 44.4\end{array}$ & $522.51(99.59)$ & $219.46(34.05)$ & $2.82(0.64)$ & $0.13(0.44)$ & $-0.57(1.52)$ & $4.37(0.72)$ \\
\hline 22 & $\begin{array}{lll}11 & 18 & 01.47\end{array}$ & $-32 \quad 4056.4$ & $857.74(81.72)$ & $299.50(31.3)$ & $6.52(0.29)$ & $2.58(0.22)$ & $3.54(1.24)$ & $1.71(0.25)$ \\
\hline 23 & $\begin{array}{lll}11 & 18 & 19.77\end{array}$ & $\begin{array}{lll}-32 & 41 & 00.9\end{array}$ & $679.36(86.05)$ & $363.79(35.10)$ & $4.11(0.65)$ & $-0.36(2.27)$ & $8.00(5.65)$ & 2.61(1.37) \\
\hline 24 & $\begin{array}{lll}11 & 18 & 19.29\end{array}$ & $-32 \quad 4053.4$ & $932.51(97.19)$ & $758.27(36.86)$ & $4.46(0.89)$ & $1.55(0.67)$ & $-9.38(2.13)$ & $-1.58(3.30)$ \\
\hline 25 & $1118 \quad 18.46$ & $-32 \quad 4100.9$ & $986.28(86.86)$ & $356.60(36.88)$ & $11.95(0.97)$ & $3.77(0.63)$ & $17.64(6.71)$ & $-8.45(2.81)$ \\
\hline 26 & 111754.82 & -324050.3 & $1133.68(84.83)$ & $438.01(28.27)$ & $13.96(0.23)$ & $7.88(0.20)$ & $-11.39(0.36)$ & $3.74(0.25)$ \\
\hline 27 & 111808.24 & $-3240 \quad 59.4$ & $1320.69(100.01)$ & $593.39(36.55)$ & $2.32(0.21)$ & $1.06(0.18)$ & $-3.96(0.43)$ & $0.80(0.31)$ \\
\hline 28 & 111800.16 & $-3241 \quad 11.4$ & $1142.09(95.43)$ & $566.99(34.87)$ & $13.86(0.22)$ & $7.89(0.17)$ & $-14.37(0.39)$ & $13.83(0.23)$ \\
\hline 29 & 111759.45 & -324133.9 & $4611.38(148.52)$ & $2402.79(47.64)$ & $4.96(0.25)$ & $2.32(0.17)$ & $-2.59(0.40)$ & $-0.03(0.66)$ \\
\hline 30 & $\begin{array}{lll}11 & 17 & 58.73\end{array}$ & $\begin{array}{lll}-32 & 42 & 05.4\end{array}$ & $521.06(89.45)$ & $183.97(37.93)$ & $10.19(0.33)$ & $4.70(0.22)$ & $-3.12(0.42)$ & $0.58(0.29)$ \\
\hline 31 & 111758.37 & $\begin{array}{lll}-32 & 42 & 18.8\end{array}$ & $2553.14(132.28)$ & $1420.85(43.65)$ & $23.95(0.35)$ & $9.71(0.23)$ & $-13.20(0.39)$ & $9.12(0.28)$ \\
\hline
\end{tabular}


Table 4-Continued

\begin{tabular}{|c|c|c|c|c|c|c|c|c|}
\hline $\begin{array}{c}\text { Association } \\
\text { ID }\end{array}$ & $\begin{array}{c}\text { RA }(J 2000) \\
\text { degree }\end{array}$ & $\begin{array}{c}\operatorname{Dec}(\mathrm{J} 2000) \\
\text { degree }\end{array}$ & $\begin{array}{l}\mathrm{FUV}^{\mathrm{a}} \\
F_{\lambda}(1 \sigma)\end{array}$ & $\begin{array}{l}\mathrm{NUV}^{\mathrm{a}} \\
F_{\lambda}(1 \sigma)\end{array}$ & $\begin{array}{c}3.6^{\mathrm{a}} \\
F_{\lambda}(1 \sigma) \\
\end{array}$ & $\begin{array}{c}4.5^{\mathrm{a}} \\
F_{\lambda}(1 \sigma) \\
\end{array}$ & $\begin{array}{c}5.8^{\mathrm{a}} \\
F_{\lambda}(1 \sigma) \\
\end{array}$ & $\begin{array}{c}8.0^{\mathrm{a}} \\
F_{\lambda}(1 \sigma) \\
\end{array}$ \\
\hline 32 & 111818.93 & -324033.9 & $2407.00(117.15)$ & $1281.40(38.02)$ & $13.90(0.71)$ & $7.03(0.48)$ & $-18.87(1.59)$ & $0.89(0.91)$ \\
\hline 34 & 111817.86 & $\begin{array}{lll}-32 & 41 & 02.4\end{array}$ & $705.34(83.42)$ & $307.99(37.92)$ & $10.33(0.92)$ & $5.44(0.57)$ & $-1.63(2.00)$ & $1.93(0.78)$ \\
\hline 35 & $\begin{array}{lll}11 & 18 & 02.89\end{array}$ & -324147.4 & $268.89(96.61)$ & $101.09(35.71)$ & $10.52(0.22)$ & $3.96(0.20)$ & $-6.37(0.40)$ & $4.39(0.26)$ \\
\hline 36 & 111811.69 & $\begin{array}{lll}-32 & 42 & 02.4\end{array}$ & $1670.35(104.11)$ & $921.23(35.63)$ & $3.77(0.52)$ & $1.52(0.43)$ & $-4.43(1.22)$ & $5.83(0.72)$ \\
\hline 37 & 111812.99 & -324154.9 & $1027.50(95.58)$ & $528.53(34.94)$ & $11.48(0.63)$ & $7.93(0.43)$ & $-34.74(1.39)$ & $-0.93(2.04)$ \\
\hline 39 & 111803.84 & $\begin{array}{lll}-32 & 42 & 23.4\end{array}$ & $667.07(85.77)$ & $222.21(30.48)$ & $7.51(0.29)$ & $2.55(0.24)$ & $-6.75(0.52)$ & $-3.67(1.04)$ \\
\hline 40 & $\begin{array}{lll}11 & 18 & 04.91\end{array}$ & $-3242 \quad 27.9$ & $1740.23(108.25)$ & $694.28(33.22)$ & $1.24(0.33)$ & $0.62(0.24)$ & $-2.40(0.53)$ & $7.64(0.41)$ \\
\hline 41 & $\begin{array}{lll}11 & 18 & 05.50\end{array}$ & $\begin{array}{lll}-32 & 42 & 27.9\end{array}$ & 1356.41(99.99) & $572.20(34.47)$ & $1.05(0.36)$ & $1.04(0.25)$ & $-7.56(0.63)$ & $0.22(0.44)$ \\
\hline 42 & $\begin{array}{lll}11 & 17 & 59.92\end{array}$ & -324245.9 & $5297.22(160.53)$ & $2714.89(51.62)$ & $31.24(0.43)$ & $12.04(0.25)$ & $-13.82(0.51)$ & $4.96(0.31)$ \\
\hline 43 & 111754.72 & -323545.8 & $1800.32(98.12)$ & $830.26(29.86)$ & $1.89(0.82)$ & $0.34(0.69)$ & $-2.95(1.90)$ & $2.21(0.78)$ \\
\hline 44 & 111755.40 & $\begin{array}{lll}-32 & 43 & 08.3\end{array}$ & $2792.85(126.27)$ & $1371.61(44.12)$ & $6.04(0.35)$ & $1.45(0.32)$ & $0.53(1.96)$ & $4.56(0.40)$ \\
\hline 45 & 111802.53 & -324256.4 & 1867.96(99.71) & $849.14(35.87)$ & $2.90(0.31)$ & $1.06(0.28)$ & $-7.37(0.61)$ & $8.30(0.42)$ \\
\hline 46 & 111752.19 & $\begin{array}{lll}-32 & 43 & 08.3\end{array}$ & $821.04(74.88)$ & $369.11(26.04)$ & $3.18(0.55)$ & $3.10(0.33)$ & $-5.55(1.00)$ & $7.09(0.47)$ \\
\hline 47 & 111806.10 & $\begin{array}{lll}-32 & 43 & 14.4\end{array}$ & $1509.85(105.78)$ & $622.26(38.02)$ & $2.62(0.53)$ & $-0.01(1.45)$ & $5.28(3.32)$ & $-16.5(2.20)$ \\
\hline 48 & 111804.67 & -324321.9 & 987.53(94.07) & $380.44(36.73)$ & $6.77(0.50)$ & $3.45(0.44)$ & $-5.23(1.10)$ & $1.57(0.67)$ \\
\hline 50 & 111754.68 & -324344.3 & $1079.12(91.93)$ & $510.94(31.09)$ & $10.05(0.63)$ & $4.01(0.49)$ & $-21.78(1.12)$ & $5.59(0.52)$ \\
\hline 51 & $\begin{array}{lll}11 & 18 & 02.29\end{array}$ & -324342.9 & $964.34(86.37)$ & $683.79(32.67)$ & $0.11(0.58)$ & $0.09(0.53)$ & $-9.33(1.07)$ & $-1.79(2.07)$ \\
\hline 52 & 111758.28 & $\begin{array}{lll}-32 & 36 & 03.8\end{array}$ & $661.66(73.92)$ & $189.50(24.97)$ & $9.18(0.37)$ & $3.63(0.36)$ & $-10.87(0.88)$ & $1.42(0.42)$ \\
\hline 53 & 111807.53 & -323851.9 & $1351.09(85.71)$ & $766.82(42.77)$ & $16.79(0.99)$ & $7.29(0.44)$ & $-4.89(0.70)$ & $-0.05(1.00)$ \\
\hline 54 & 111800.52 & $-32 \quad 38 \quad 57.9$ & $384.90(79.55)$ & $253.36(31.27)$ & $2.08(0.21)$ & $0.61(0.20)$ & $2.98(1.21)$ & $-2.42(0.72)$ \\
\hline 55 & $\begin{array}{lll}11 & 18 & 01.71\end{array}$ & $\begin{array}{lll}-32 & 39 & 09.9\end{array}$ & $624.68(77.51)$ & $198.65(30.36)$ & $9.47(0.27)$ & $5.09(0.20)$ & $-9.88(0.40)$ & $9.24(0.27)$ \\
\hline 56 & 111801.59 & -323924.9 & 1229.37(93.21) & $640.69(34.75)$ & $20.69(0.49)$ & $13.70(0.29)$ & $-17.87(0.43)$ & $6.77(0.28)$ \\
\hline 57 & 111757.08 & $\begin{array}{lll}-32 & 39 & 24.8\end{array}$ & $4924.55(143.85)$ & $2594.04(42.67)$ & $18.21(0.20)$ & $6.86(0.18)$ & $-11.22(0.37)$ & $6.14(0.23)$ \\
\hline 58 & $11 \quad 1757.32$ & -323930.8 & $4003.19(131.79)$ & $2004.32(40.82)$ & $9.59(0.20)$ & $3.71(0.18)$ & $-2.25(0.38)$ & $1.76(0.23)$ \\
\hline \multicolumn{9}{|c|}{$\underline{\text { NGC } 5055 \text { Associations }}$} \\
\hline 1 & 131620.50 & +414835.6 & $2395.78(172.20)$ & $1375.27(53.16)$ & $107.81(0.50)$ & $47.89(0.33)$ & $-130.26(1.04)$ & $253.88(0.59)$ \\
\hline 2 & 131623.49 & +415331.0 & $1140.49(133.17)$ & $303.89(38.03)$ & $10.45(0.34)$ & $6.44(0.27)$ & $-20.67(0.71)$ & $5.82(0.36)$ \\
\hline 3 & 131607.90 & +415307.3 & $1113.16(132.19)$ & $100.95(37.20)$ & $57.49(0.41)$ & $22.22(0.34)$ & $-17.13(1.24)$ & $14.79(0.48)$ \\
\hline 4 & 131609.12 & +415326.7 & $1782.40(154.37)$ & $516.30(39.63)$ & $38.17(0.54)$ & $21.41(0.38)$ & $-34.56(1.24)$ & $11.57(0.46)$ \\
\hline 5 & 131622.00 & +415223.6 & 1249.06(136.99) & $249.07(34.63)$ & $17.26(0.28)$ & $8.77(0.22)$ & $0.65(1.96)$ & $8.68(0.34)$ \\
\hline 6 & 131606.83 & +415222.3 & $1216.54(135.86)$ & $205.38(34.39)$ & $4.36(0.40)$ & $2.29(0.36)$ & $17.24(4.31)$ & $7.85(0.48)$ \\
\hline 7 & 131624.29 & +415241.5 & $1021.85(128.87)$ & $411.50(35.77)$ & $33.11(0.30)$ & $15.55(0.24)$ & $-19.91(0.63)$ & $12.23(0.34)$ \\
\hline 11 & 131620.11 & +415122.1 & $2131.99(164.77)$ & $886.75(47.02)$ & $67.24(0.41)$ & $27.75(0.28)$ & $-35.63(0.63)$ & $13.00(0.34)$ \\
\hline 12 & 131610.98 & +415019.2 & $1380.56(141.47)$ & $756.48(45.30)$ & $22.64(0.23)$ & $18.91(0.26)$ & $-45.04(0.67)$ & $28.59(0.34)$ \\
\hline 13 & 131625.75 & +415122.0 & $1135.79(133.00)$ & $277.14(38.06)$ & $22.33(0.37)$ & $10.48(0.27)$ & $-15.96(0.56)$ & $7.7(0.32)$ \\
\hline 14 & 131626.96 & +415122.0 & $991.30(127.74)$ & $124.03(31.99)$ & $1.13(0.32)$ & $0.46(0.27)$ & $-4.91(0.59)$ & $0.99(0.36)$ \\
\hline 15 & 131627.89 & +414955.0 & $659.25(114.74)$ & $264.63(33.33)$ & $23.07(0.29)$ & $12.01(0.29)$ & $-23.10(0.63)$ & $3.67(0.38)$ \\
\hline 16 & 131633.85 & +415445.8 & $940.66(125.85)$ & $134.95(30.48)$ & $4.39(0.50)$ & $1.49(0.46)$ & $-16.62(1.51)$ & $4.48(0.67)$ \\
\hline 17 & $\begin{array}{lll}13 & 16 & 17.14\end{array}$ & +414852.1 & $849.34(122.35)$ & $234.32(35.46)$ & $10.39(0.39)$ & $4.7(0.31)$ & $9.19(2.46)$ & $1.33(0.47)$ \\
\hline
\end{tabular}

Note. - (a) Fluxes are in units of $10^{-19} \mathrm{ergs} \mathrm{s}^{-1} \mathrm{~cm}^{-2} \mathrm{~A}^{-1}$; the $1 \sigma$ uncertainties are indicated in parenthesis, following the flux value. For FUV and NUV band, the flux has been corrected for the foreground Milky Way extinction. 
To quantify the remaining background galaxy contamination, we derived the number of potential contaminants within the region of interest ( $\sim 1$ square arcminute). Our deepest observations are of NGC 3621, with a 3 sigma FUV detection limit corresponding to an $\mathrm{AB}$ magnitude of 25.9. Taking into account that objects larger than 2" will be resolved by IRAC and recognizable as extended sources, we can estimate the number of point sources within 1 square degree to be $\sim 10^{4}$ (Xu et al. 2005). This leaves us with a conservative $3-5$ galaxies per square arcminute for NGC 3621, and less for our other shallower galaxies. In reality, the number of contaminating galaxies is even less than our already conservative estimate. Most galaxies are redder on average, due to older stellar populations or larger dust content, than the associations of interest here. These galaxies are automatically discarded through Equation (1) because of their faint FUV emission. Furthermore, dusty low redshift galaxies would present excess $8.0 \mu \mathrm{m}$ emission from Polycyclic Aromatic Hydrocarbons (PAH) in the IRAC4 band (e.g. (Leger \& Puget 1984; Smith et al. 2007)) which we do not detect in our images (see Section 4.1). This analysis indicates that the level of background galaxy contamination in our sample is low and we neglect it in what follows.

Our final number of sources is 229. Their locations, fluxes, and uncertainties are listed in Table 4. Figures 2-6 show the star forming associations on the FUV, NUV, $3.6 \mu \mathrm{m}$ and $8.0 \mu \mathrm{m}$ images of each galaxy. 

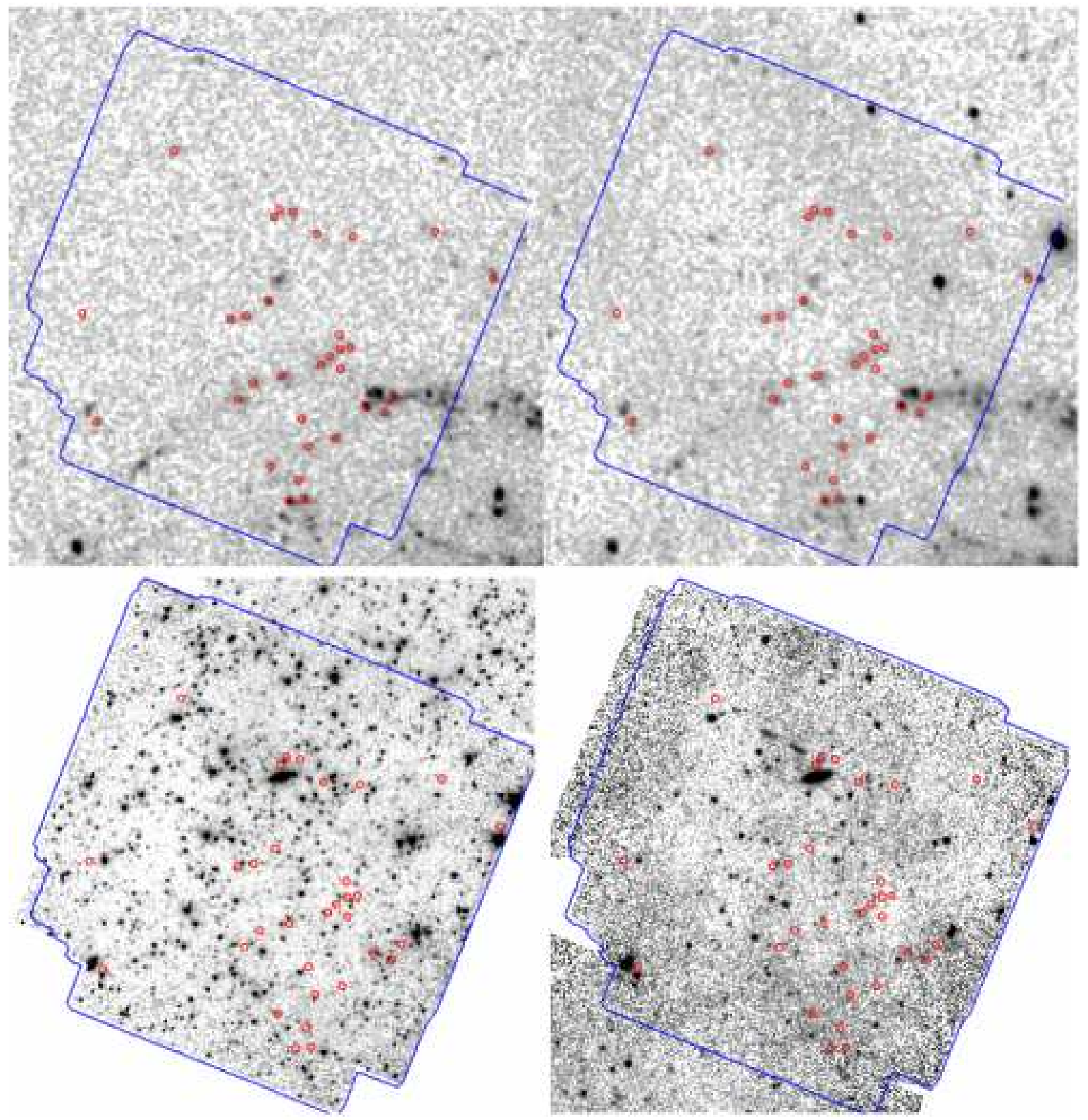

Fig. 2.- GALEX FUV (top left) and NUV (top right) and Spitzer $3.6 \mu \mathrm{m}$ (bottom left) and $8.0 \mu \mathrm{m}$ (bottom right) images of the target region in NGC 0628. There are $32 \mathrm{UV}$-bright star forming associations which are indicated by the red apertures used in measuring the photometry (see Table 3 for aperture sizes). UV sources that are not selected are either below our detection threshold, discarded due to missing the $3.6 \mu \mathrm{m}$ band, or candidates for foreground stars or background galaxies. 


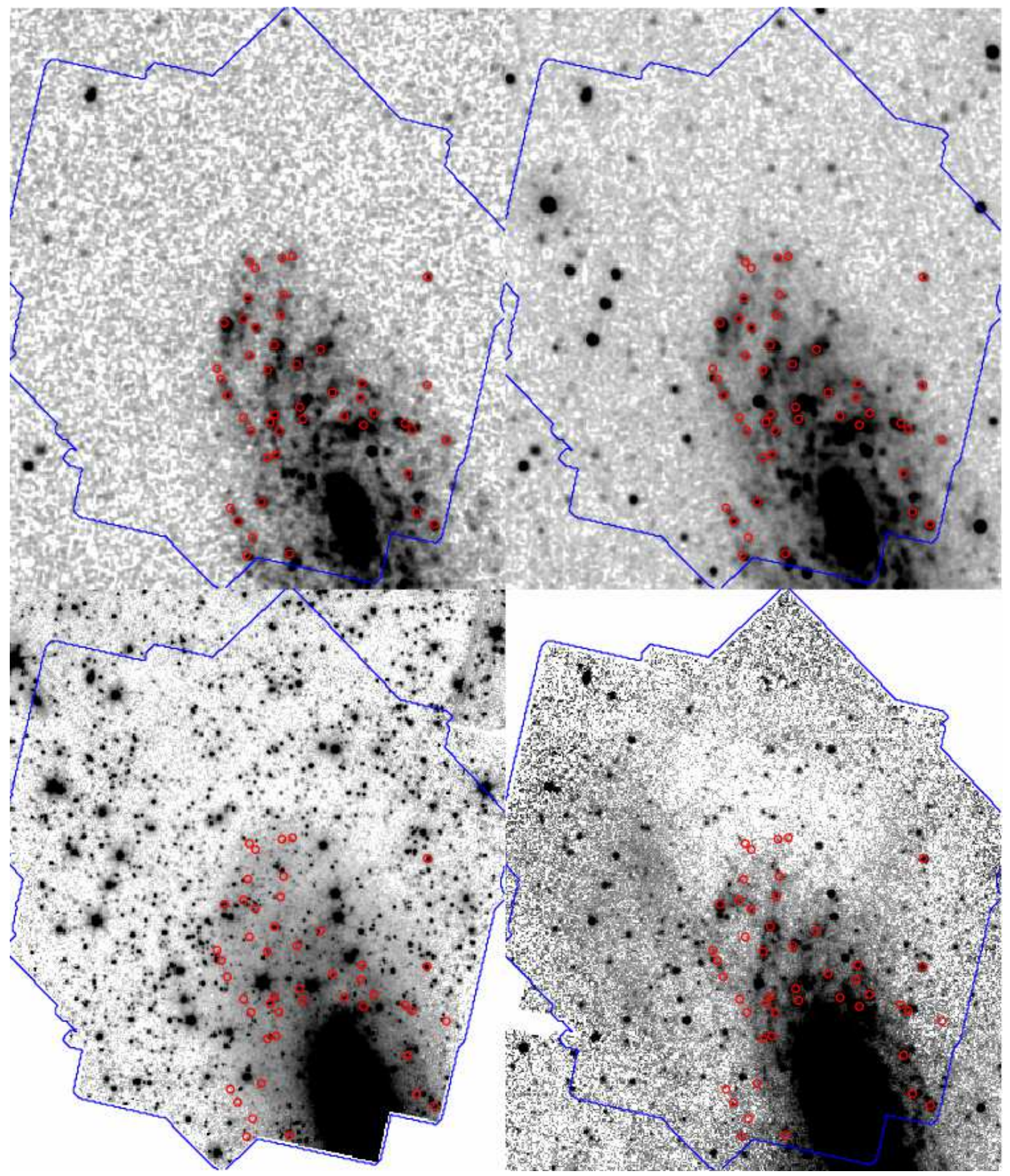

Fig. 3.- As in Figure 2, for NGC 2090. There are 47 final UV-bright associations. 


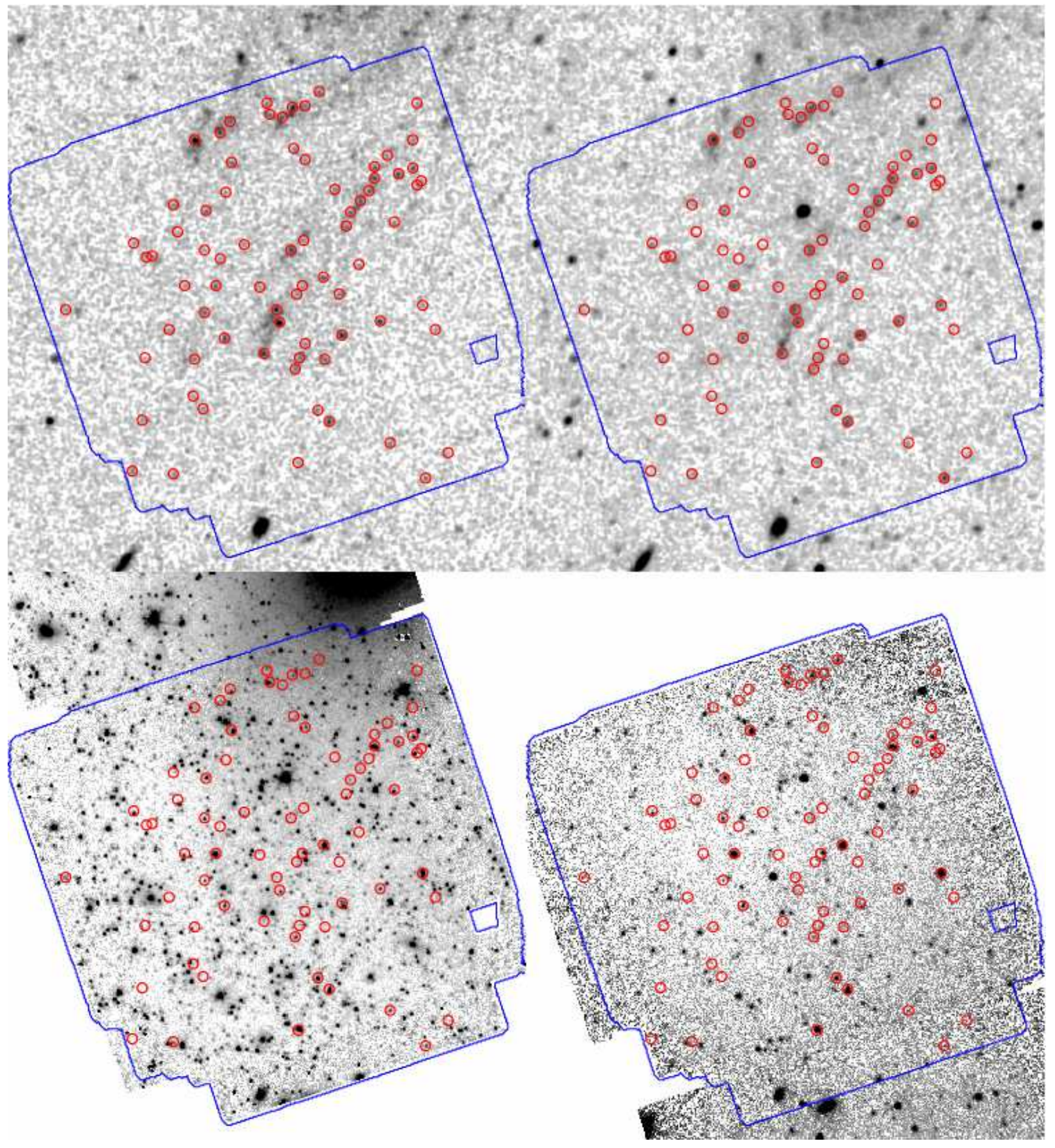

Fig. 4.- As in Figure 2, for NGC 2841. There are 75 final UV-bright associations. 


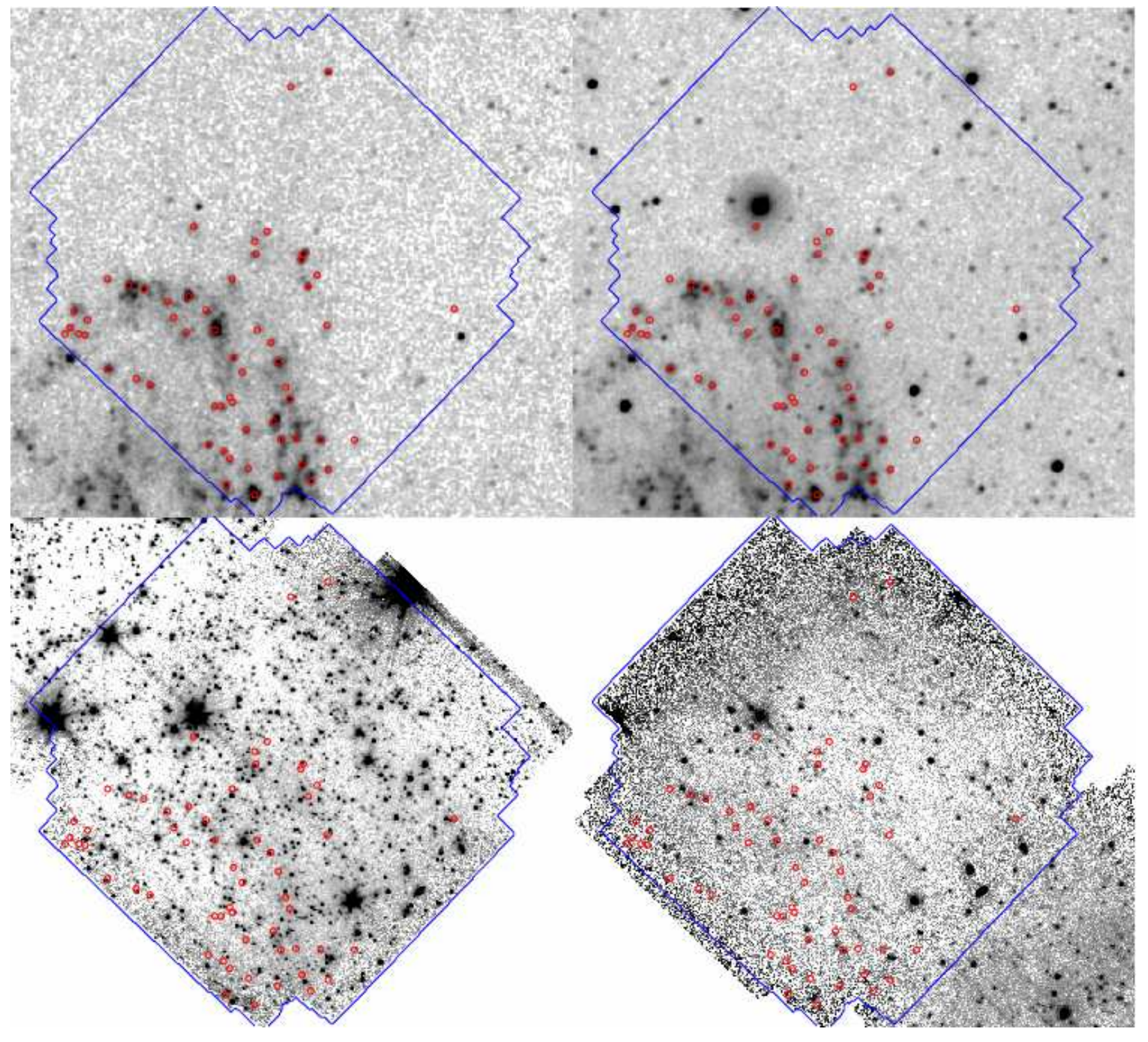

Fig. 5.- As in Figure 2, for NGC 3621. There are 57 final UV-bright associations. 


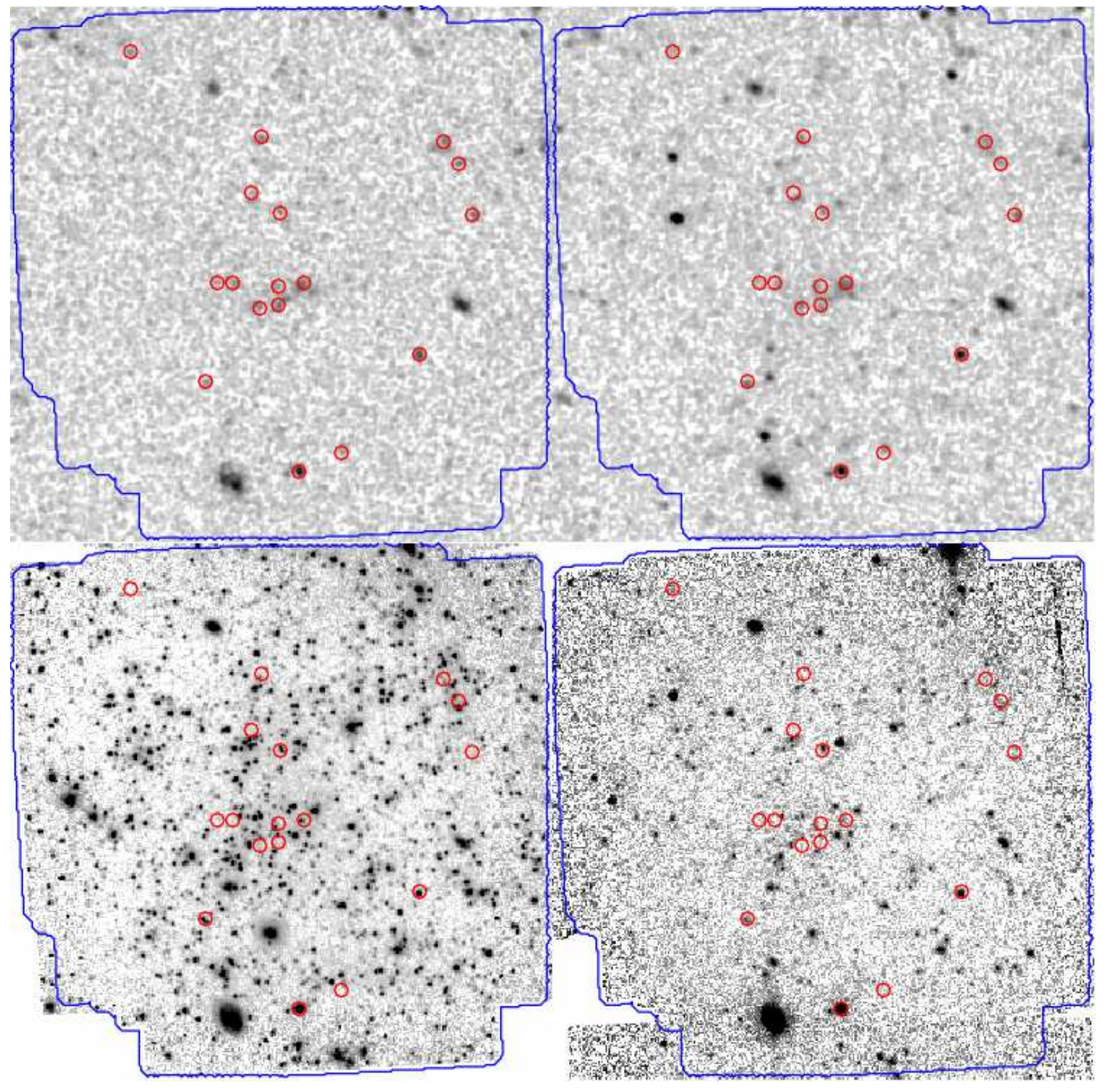

Fig. 6.- As in Figure 2, for NGC 5055. There are only 17 final associations, due to intrinsically high noise levels (see Section 5.5). 


\section{Analysis}

\subsection{Stellar Population Modeling}

To constrain the age and mass of each source we fit their UV+MIR SED with a model SED of a single age stellar population. These model SEDs are a combination of nebular and stellar continuum emission and, as such, do not account for the effects of extinction, i.e. the redistribution of ultraviolet light into the infrared by dust. We can make the reasonable

assumption that the $3.6 \mu \mathrm{m}$ and $4.5 \mu \mathrm{m}$ bands are free of this effect (Pahre et al. 2004; Calzetti et al. 2005; Engelbracht et al. 2008). This is not in general true for the $5.8 \mu \mathrm{m}$ and $8.0 \mu \mathrm{m}$ bands as they may contain a significant amount of dust emission including that of polycyclic aromatic hydrocarbons (PAHs). As such, they will not be directly included in our fits, leaving us with four bands.

To further address the question of dust, we note that Gil de Paz et al. (2007a) found sub-solar metallicities for the HII regions in the outer disk of M83 with $\mathrm{Z} / \mathrm{Z}_{\odot}=1 / 5-1 / 10$. Coupled with the result that low metallicity regions are expected to also have under-luminous $8.0 \mu \mathrm{m}$ dust emission (Engelbracht et al. 2005, 2008; Calzetti et al. 2007; Draine et al. 2007), we infer that outer disks will have little dust in general. To see if this is true for our galaxies and our particular associations, we create dust maps by taking the $8.0 \mu \mathrm{m}$ image and subtracting off a scaled $3.6 \mu \mathrm{m}$ image (which represents the continuum). In general, these dust maps show weak or null dust detections near our associations, which is in agreement with the low metallicity values. This will be discussed in more detail for each galaxy in Section 5 .

All of our fits are done using Starburst99 stellar population synthesis models (Leitherer et al. 1999; Vazquez et al. 2005). Our associations are small (60-110 pc) and therefore most likely contain a single or a few stellar clusters, which we model as instantaneous burst populations with sub-solar metallicities $(\mathrm{Z}=0.004)$. Comparisons between sub-solar and solar metallicity models show only negligible differences at the wavelengths utilized in this study. Initially the IMF (see also Section 6) is chosen to be the default Kroupa IMF, which is expressed by a broken power law $\left(\phi(\mathrm{M}) \propto \mathrm{M}^{-\alpha}\right)$ with a slope of $\alpha=1.3$ for $0.1 M_{\odot}<M<0.5 M_{\odot}$ and $\alpha=2.3$ for $0.5 M_{\odot}<M<100 M_{\odot}$ (Kroupa et al. 2001). We employ the Padova tracks with full asymptotic giant branch (AGB) evolution, the latter to better fit the MIR fluxes. The contribution by thermally-pulsating AGB stars should be minimal for associations much younger than 1 Gyr (Maraston et al. 2006). Models are generated in timesteps of 1 Myr for 1-10 Myr and $5 \mathrm{Myr}$ for $10 \mathrm{Myr}-1 \mathrm{Gyr}$ and then convolved with the GALEX and IRAC filter bandpasses to create the final synthetic photometry. 


\subsection{Age and Mass Fitting}

Observational data are fit with the synthetic photometry from models using $\chi^{2}$-minimization (Dong et al. 2008):

$$
\chi^{2}(t, E(B-V), M, Z)=\sum_{N} \frac{\left(L_{o b s}-A \times L_{\text {model }}\right)^{2}}{\sigma_{o b s}^{2}}
$$

where $\mathrm{N}$ is the number of filters per source, $L_{o b s}$ is the observed luminosity, $L_{\text {model }}$ is the model luminosity, and $\sigma_{o b s}$ is the measurement uncertainty. Effectively the age of each association is found by comparing the shape of each association's SED to the shape of each model, i.e. the intensity in UV relative to the intensity in the IR bands. The mass is an $a$ priori unknown scaling factor which is represented by the dimensionless parameter $A$, where $A=\frac{M}{10^{6} M_{\odot}}, M$ is the mass in the aperture, and $10^{6} M_{\odot}$ is the default mass of the Starburst99 models. The underlying stellar components (and thus the mass) of an association are best traced by the $3.6 \mu \mathrm{m}$ emission and so we use this as our guideline to find the mass scaling factor; however, we do not fix the mass to this point, but allow the scaling factor to vary within the $3.6 \mu \mathrm{m} 1 \sigma$ errors during fitting. In the few cases of very large errors, we also impose a minimum detectable mass derived by combining the FUV $3 \sigma$ detection threshold with a 1 Myr stellar population model, our bluest synthetic SED. A good fit (within the $1 \sigma$ errors) is shown in Figure 7.

The age-extinction degeneracy prevents us from fitting for age and extinction simultaneously. Though we have reason to believe (as outlined above) that these regions are low-dust and low-metallicity, we generate fits for two fixed extinctions (in addition to foreground Galactic extinction, hereafter implied): $E(B-V)=0$ and $E(B-V)=0.3$, the latter being the average extinction value for inner disks and the high end of the extinction values observed in the outer disk of M83 (Gil de Paz et al. 2007a). Extinction is applied using Milky Way-type dust with $A_{F U V} / E(B-V)=8.38$ and $A_{N U V} / E(B-V)=8.74$ (Wyder et al. 2005). The actual extinction is specific to a given association and difficult to ascertain with the available information. However, by analyzing both extreme fits, we can make determinations about the relative extinction of these galaxies as a whole and make predictions about the 'best fit' for a given association within a set of reasonable solutions. In general, we also note that higher extinction values are found for younger associations and lower extinctions for older associations. We also fit the associations in our most extincted galaxy (NGC 2841, see Section 5.4) using the starburst attenuation curve of Calzetti et al. (2000), but find that the results are similar to those obtained using the Milky Way extinction curve plus foreground dust. We ascribe this to the small extinction values that characterize our regions.

The age and mass of each association are listed in Table 5, with associations separated 
for each galaxy into two groups: those better fit by $E(B-V)=0$ and those better fit by $E(B-V)=0.3$. The $1 \sigma$ uncertainties were determined by generating age/mass confidence intervals for each association. An example of a typical confidence interval (at 68\%, 90\%, and $99 \%$ levels) can be seen in Figure 8. It should be noted that, because the mass is tied to the value of the $3.6 \mu \mathrm{m}$ datapoint, increasing the mass will result in a decreased UV to MIR ratio, which corresponds to an older age. In other words, there is a positive correlation between age and mass.
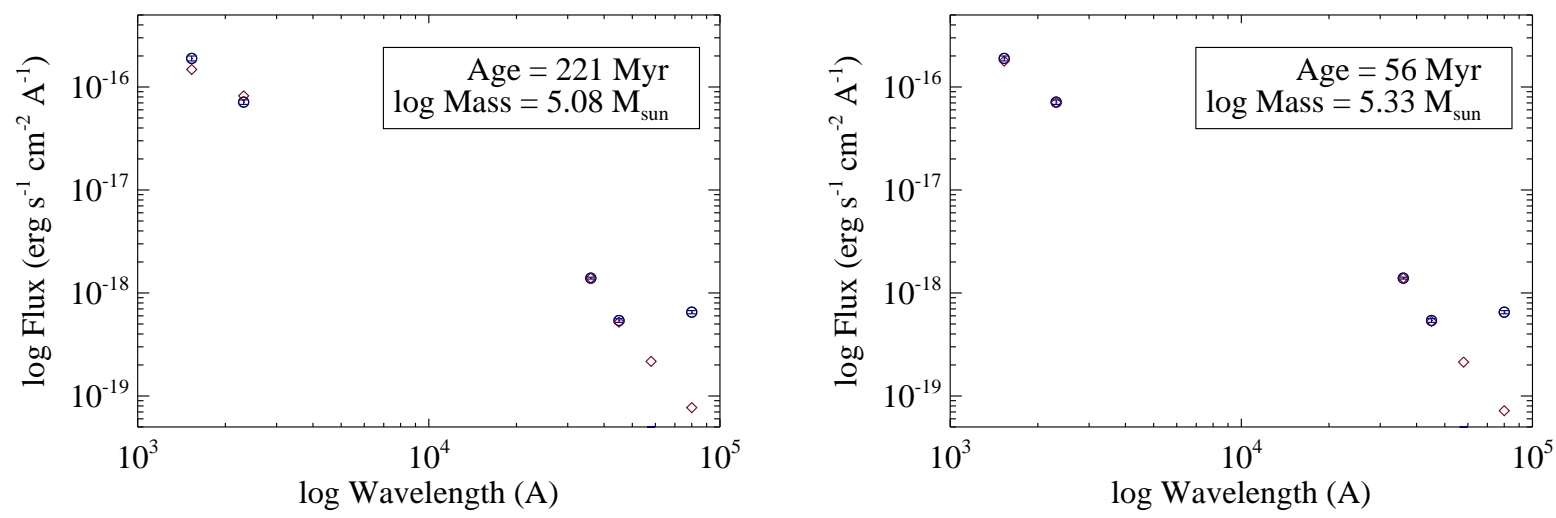

Fig. 7.- Association 13 in NGC 3621 is a good example of an association that is well fit in both the no dust (left) and $E(B-V)=0.3$ (right) cases. The circles (blue) with error bars represent the datapoints from this association and the diamonds (red) represent the model points. The 5.8 and $8.0 \mu \mathrm{m}$ points are not used in any fits due to PAH emission contamination. In this particular case, the $5.8 \mu \mathrm{m}$ emission is not detected. 

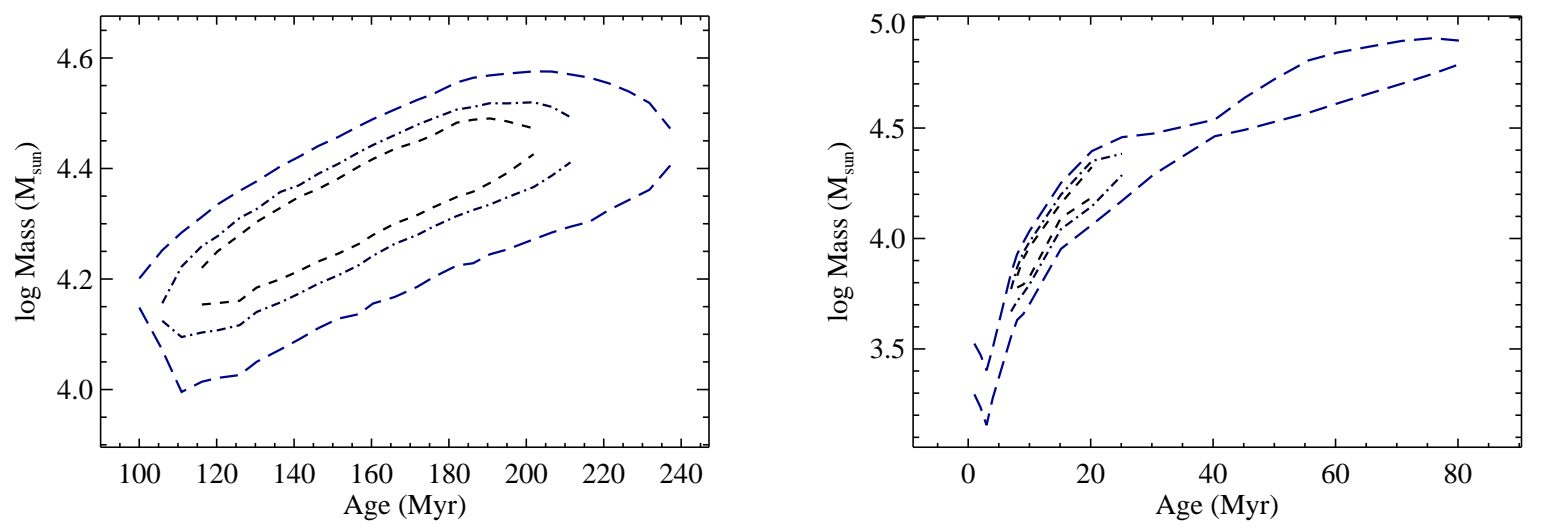

Fig. 8.- Confidence contours for association 21 in NGC 3621 with $E(B-V)=0.0$ (left) and $E(B-V)=0.3$ (right). This association's best fit age is $\sim 166 \mathrm{Myr}$ and, in terms of dust content, is not significantly different from the other associations in this or other galaxies. The short dashed black line is at a $68 \%$ confidence level, the dot dashed purple line at $90 \%$, and the long dashed blue line is $99 \%$. 
Table 5. Derived Ages and Masses for All Associations

\begin{tabular}{|c|c|c|c|c|}
\hline \multirow{2}{*}{$\begin{array}{c}\text { Association } \\
\text { ID }\end{array}$} & \multicolumn{2}{|c|}{ Age (Myr) } & \multicolumn{2}{|c|}{$\log \operatorname{Mass}\left(M_{\odot}\right)$} \\
\hline & $E(B-V)=0.0$ & $E(B-V)=0.3$ & $E(B-V)=0.0$ & $E(B-V)=0.3$ \\
\hline \multicolumn{5}{|c|}{$\underline{\text { NGC } 0628 \text { Associations }}$} \\
\hline \multicolumn{5}{|c|}{ Best Fit with No Dust } \\
\hline 3 & $989_{-5}^{+>11}$ & $116_{-5}^{+5}$ & $5.82_{-0.01}^{+0.01}$ & $5.28_{-0.00}^{+0.03}$ \\
\hline 5 & $90_{-40}^{+10}$ & $6_{-1}^{+1}$ & $4.02_{-0.10}^{+0.10}$ & $3.53_{-0.17}^{+0.00}$ \\
\hline 8 & $120_{-4}^{+16}$ & $15_{-5}^{+5}$ & $4.46^{+0.04}$ & $4.39^{+0.03}$ \\
\hline 12 & $35_{-5}^{+60}$ & $6_{-1}^{+-5}$ & $4.40_{-0.50}^{+0.50}$ & $\begin{array}{r}-0.04 \\
4.41_{-0.03}^{+0.03}\end{array}$ \\
\hline 14 & $106_{-5}^{+5}$ & $7_{-3}^{\overline{+}+1}$ & $4.57_{-0.03}^{+0.03}$ & $4.10_{-0.34}^{+0.03}$ \\
\hline $15^{\mathrm{a}}$ & $1_{-1}^{+29^{-5}}$ & $6_{-1}^{+3}$ & $2.72_{-1.06}^{+1.06}$ & $\begin{array}{l}-0.34 \\
3.82_{-0.04}^{+0.04}\end{array}$ \\
\hline 17 & $65_{-45}^{+25}$ & $6_{-1}^{+1}$ & $4.06_{-0.17}^{+0.17}$ & $3.73_{-0.07}^{+0.06}$ \\
\hline 18 & $881_{-59}^{+74}$ & $111_{-5}^{+5}$ & $6.06_{-0.03}^{+0.03}$ & $5.60_{-0.01}^{+0.01}$ \\
\hline $19^{\mathrm{a}}$ & $20_{-19}^{+\overline{5} 6}$ & $6_{-1}^{+1}$ & $3.58_{-0.68}^{+0.68}$ & $3.87_{-0.14}^{+0.01}$ \\
\hline 23 & $35_{-10}^{+19}$ & $6_{-1}^{+1}$ & $4.10^{+0.18}$ & $4.11_{-0.12}^{+0.14}$ \\
\hline 24 & $60_{-45}^{-10}$ & $6_{-1}^{+1}$ & $4.14_{-0.20}^{+0.18}$ & $3.88_{-0.06}^{+0.12}$ \\
\hline 25 & $\begin{array}{l}-45 \\
20_{-19}^{+60}\end{array}$ & $6_{-1}^{+1}$ & $\begin{array}{l}-0.20 \\
3.52_{-0.71}^{+0.71}\end{array}$ & $\begin{array}{r}-0.06 \\
3.81_{-0.06}^{+0.05}\end{array}$ \\
\hline 26 & $822_{-9}^{+166}$ & $111_{-5}^{+5}$ & $5.81_{-0.07}^{+0.07}$ & $5.37_{-0.04}^{+0.06}$ \\
\hline 28 & $126_{-15}^{+56}$ & $9_{-8}^{+6}$ & $4.21_{-0.18}^{+0.18}$ & $3.82_{-0.58}^{+0.045}$ \\
\hline 30 & $191_{-39}^{+16}$ & $1_{-1}^{+1}$ & $4.49_{-0.05}^{+0.05}$ & $3.50_{-0.06}^{+0.05}$ \\
\hline 31 & $725_{-187}^{+43}$ & $96_{-5}^{+5}$ & $5.70_{-0.03}^{+0.03}$ & $5.50_{-0.01}^{+0.00}$ \\
\hline 32 & $116_{-5}^{+4}$ & $15_{-8}^{+5}$ & $4.67_{-0.02}^{+0.02}$ & $4.61_{-0.39}^{+0.02}$ \\
\hline \multicolumn{5}{|c|}{ Best Fit with Dust } \\
\hline $1^{\mathrm{a}}$ & $35_{-5}^{+65}$ & $6_{-1}^{+1}$ & $4.29^{+0.51}$ & $4.31_{-0.13}^{+0.03}$ \\
\hline $2^{\mathrm{b}}$ & $211^{-5}+10$ & $25^{-1}+5$ & $4.69^{+0.03}$ & $4.57^{-0.02}$ \\
\hline $4^{\mathrm{b}}$ & $1_{-1}^{+226}$ & $2_{-1}^{+23}$ & $1.98_{-2.07}^{+0.03}$ & $2.94_{-0.28}^{+0.02}$ \\
\hline $6^{\mathrm{b}}$ & $211_{-16}^{+20}$ & $25_{-5}^{+46}$ & $4.49_{-0.05}^{+0.05}$ & $4.37_{-0.04}^{+0.45}$ \\
\hline 7 & $562_{-290}^{+61}$ & $100_{-5}^{+5}$ & $5.37_{-0.02}^{+0.05}$ & $5.22_{-0.05}^{+0.04}$ \\
\hline $9^{\mathrm{b}}$ & $211^{+5}$ & $25^{+-50}$ & $4.83^{+0.03}$ & $4.70^{+0.42}$ \\
\hline 10 & $237_{-65}^{+665}$ & $30_{-20}^{+8}$ & $3.91_{-0.64}^{+0.63}$ & $3.88_{-0.51}^{+0.02}$ \\
\hline $11^{\mathrm{b}}$ & $202_{-5}^{+55}$ & $1_{-1}^{+\overline{1}^{20}}$ & $5.05_{-0.00}^{+0.00}$ & $4.15_{-0.01}^{+0.01}$ \\
\hline $13^{\mathrm{b}}$ & $186_{-5}^{+5}$ & $1_{-1}^{+1}$ & $5.48_{-0.00}^{+0.00}$ & $4.54_{-0.00}^{+0.00}$ \\
\hline 16 & $617_{-41}^{+133}$ & $100_{-5}^{+6}$ & $5.65_{-0.11}^{+0.11}$ & $5.50_{-0.08}^{+0.01}$ \\
\hline $20^{\mathrm{b}}$ & $211_{-5}^{-41}$ & $56_{-30}^{+15}$ & $4.81_{-0.02}^{+0.11}$ & $5.03_{-0.36}^{+0.08}$ \\
\hline 21 & $989_{-264}^{+>11}$ & $111_{-5}^{+9}$ & $5.49_{-0.02}^{+0.02}$ & $4.99_{-0.05}^{+0.09}$ \\
\hline 22 & $302_{-51}^{+49}$ & $35_{-5}^{+65}$ & $4.67_{-0.14}^{+0.14}$ & $4.59_{-0.04}^{+0.44}$ \\
\hline 27 & $1000_{-11}^{+1}>5$ & $126_{-5}^{+15}$ & $6.08_{-0.00}^{+0.00}$ & $5.48_{-0.00}^{+0.02}$ \\
\hline 29 & $126_{-125}^{+250}$ & $3_{-2}^{+22}$ & $3.66_{-0.36}^{+0.36}$ & $2.80_{-0.21}^{+0.98}$ \\
\hline \multicolumn{5}{|c|}{ NGC 2090 Associations } \\
\hline & & Best Fit with No & & \\
\hline 2 & $313_{-11}^{+5}$ & $96_{-5}^{+5}$ & $6.26_{-0.00}^{+0.00}$ & $6.54_{-0.00}^{+0.00}$ \\
\hline 3 & $146_{-10}^{+11}$ & $1_{-1}^{+\overline{8}^{5}}$ & $5.46_{-0.04}^{+0.04}$ & $4.58_{-0.06}^{+0.00}$ \\
\hline 4 & $126_{-5}^{+\frac{1}{+5}}$ & $9_{-1}^{+1}$ & $5.57_{-0.03}^{+0.03}$ & $5.18_{-0.01}^{+0.06}$ \\
\hline 6 & $106_{-5}^{+5}$ & $7_{-1}^{+1}$ & $5.90_{-0.00}^{+0.00}$ & $5.38_{-0.00}^{+0.00}$ \\
\hline 7 & $989_{-45}^{+5} 11$ & $116_{-5}^{+14}$ & $6.25_{-0.02}^{+0.02}$ & $5.71_{-0.01}^{+0.00}$ \\
\hline 8 & $166_{-15}^{+16}$ & $10_{-1}^{+1^{5}}$ & $5.62_{-0.05}^{+0.05}$ & $\begin{array}{r}5.14_{-0.02}^{+0.02} \\
0.02\end{array}$ \\
\hline 9 & $100_{-5}^{+5}$ & $6_{-1}^{+1}$ & $5.41_{-0.02}^{+0.02}$ & $4.90_{-0.02}^{+0.03}$ \\
\hline 10 & $186_{-5}^{+5}$ & $20_{-5}^{+5}$ & $5.97_{-0.01}^{+0.01}$ & $5.79_{-0.01}^{+0.01}$ \\
\hline 12 & $106_{-6}^{+5}$ & $6_{-1}^{+-5}$ & $5.26_{-0.03}^{+0.03}$ & $4.73_{-0.12}^{+0.01}$ \\
\hline 13 & $141_{-15}^{+25}$ & $2_{-1}^{+\frac{1}{7}}$ & $5.45_{-0.08}^{+0.08}$ & $4.52_{-0.03}^{+0.150}$ \\
\hline 16 & $989_{-5}^{+>11}$ & $116_{-5}^{+5}$ & $6.52_{-0.01}^{+0.01}$ & $5.98_{-0.00}^{+0.00}$ \\
\hline 17 & $100_{-5}^{+5}$ & $6_{-1}^{+1^{-5}}$ & $5.24_{-0.02}^{+0.02}$ & $4.76_{-0.02}^{+0.02}$ \\
\hline 19 & $56_{-30}^{+30}$ & $6_{-1}^{-1}$ & $4.92_{-0.22}^{+0.02}$ & $4.71_{-0.03}^{+0.02}$ \\
\hline 21 & $191_{-5}^{+5}$ & $20_{-5}^{+5}$ & $5.71_{-0.02}^{+0.02}$ & $5.53_{-0.02}^{+0.01}$ \\
\hline 22 & $111_{-5}^{+5}$ & $7_{-1}^{+1}$ & $5.82_{-0.01}^{+0.01}$ & $5.39_{-0.01}^{+0.01}$ \\
\hline
\end{tabular}


Table 5-Continued

\begin{tabular}{|c|c|c|c|c|}
\hline \multirow{2}{*}{$\begin{array}{c}\text { Association } \\
\text { ID }\end{array}$} & \multicolumn{2}{|c|}{ Age (Myr) } & \multicolumn{2}{|c|}{$\log \operatorname{Mass}\left(M_{\odot}\right)$} \\
\hline & $E(B-V)=0.0$ & $E(B-V)=0.3$ & $E(B-V)=0.0$ & $E(B-V)=0.3$ \\
\hline 30 & $111_{-5}^{+5}$ & $7_{-1}^{+8}$ & $5.06_{-0.02}^{+0.02}$ & $4.62_{-0.03}^{+0.40}$ \\
\hline 33 & $96_{-5}^{+5}$ & $6_{-1}^{+1}$ & $5.34_{-0.02}^{+0.02}$ & $4.85_{-0.02}^{+0.02}$ \\
\hline 34 & $111_{-5}^{+5}$ & $15_{-5}^{+5}$ & $4.96_{-0.03}^{+0.03}$ & $4.91_{-0.04}^{+0.02}$ \\
\hline $35^{\mathrm{a}}$ & $20_{-14}^{+\overline{5}^{5}}$ & $6_{-1}^{+\overline{1}^{5}}$ & $4.42_{-0.02}^{+0.03}$ & $4.50_{-0.00}^{+0.04}$ \\
\hline 39 & $989_{-11}^{+>11}$ & $126_{-126}^{+126}$ & $6.70_{-0.01}^{+0.01}$ & $6.11_{-0.00}^{+0.00}$ \\
\hline 41 & $130_{-10}^{+5}$ & $8_{-1}^{+1}$ & $5.22_{-0.02}^{+0.02}$ & $4.82_{-0.02}^{+0.02}$ \\
\hline 46 & $141_{-5}^{+10}$ & $9_{-1}^{+1}$ & $5.64_{-0.04}^{+0.04}$ & $5.21_{-0.02}^{+0.01}$ \\
\hline \multicolumn{5}{|c|}{ Best Fit with Dust } \\
\hline 1 & $1000_{-5}^{+>5}$ & $136_{-6.0}^{+10}$ & $7.10_{-0.00}^{+0.00}$ & $6.53_{-0.00}^{+0.01}$ \\
\hline $5^{\mathrm{b}}$ & $120_{-5}^{+10}$ & $3_{-1}^{+1}$ & $4.99_{-0.02}^{+0.02}$ & $4.09_{-0.03}^{+0.03}$ \\
\hline 11 & $1000_{-11}^{+}>5$ & $120_{-5}^{+5}$ & $6.83_{-0.00}^{+0.00}$ & $6.28_{-0.00}^{+0.01}$ \\
\hline $14^{\mathrm{b}}$ & $202_{-5}^{+5}$ & $20_{-5}^{+5}$ & $6.03_{-0.00}^{+0.00}$ & $5.86_{-0.01}^{+0.01}$ \\
\hline $15^{\mathrm{b}}$ & $211_{-5}^{+10}$ & $60_{-5}^{+11}$ & $5.64_{-0.02}^{+0.02}$ & $5.9_{-0.06}^{+0.07}$ \\
\hline 18 & $989_{-194}^{+>11}$ & $116_{-5.0}^{+41}$ & $5.91_{-0.04}^{+0.04}$ & $5.38_{-0.08}^{+0.06}$ \\
\hline $20^{\mathrm{b}}$ & $195_{-5}^{+\frac{1}{5}}$ & $20_{-5}^{+\overline{5}^{5.0}}$ & $5.64_{-0.02}^{+0.02}$ & $5.47_{-0.02}^{+0.02}$ \\
\hline $23^{\mathrm{b}}$ & $207_{-5}^{+5}$ & $25_{-5}^{+5}$ & $5.86_{-0.01}^{+0.01}$ & $5.74_{-0.01}^{+0.00}$ \\
\hline 24 & $246_{-19}^{+298}$ & $85_{-50}^{+5}$ & $5.03_{-0.49}^{+0.01}$ & $5.45_{-0.47}^{+0.01}$ \\
\hline 25 & $216_{-5}^{+10}$ & $1_{-5}^{+\overline{5}^{50}}$ & $5.25_{-0.01}^{+0.09}$ & $4.32_{-0.03}^{+0.03}$ \\
\hline 26 & $126_{-5}^{+15}$ & $8_{-5}^{+1}$ & $5.15_{-0.06}^{+0.06}$ & $4.77_{-0.53}^{+0.04}$ \\
\hline 27 & $257_{-6}^{+274}$ & $90_{-5}^{+5}$ & $5.63_{-0.47}^{+0.47}$ & $6.03_{-0.01}^{+0.01}$ \\
\hline $28^{\mathrm{b}}$ & $211_{-5}^{+5}$ & $25_{-5}^{+30}$ & $5.54_{-0.02}^{+0.02}$ & $5.42_{-0.02}^{+0.36}$ \\
\hline 29 & $232_{-5}^{+5}$ & $76_{-5}^{+5}$ & $5.72_{-0.02}^{+0.02}$ & $6.11_{-0.04}^{+0.02}$ \\
\hline 31 & $195_{-5}^{+7}$ & $20_{-5}^{+5}$ & $5.69_{-0.02}^{+0.02}$ & $5.52_{-0.02}^{+0.04}$ \\
\hline $32^{\mathrm{b}}$ & $126_{-5}^{+5}$ & $3_{-1}^{+1^{5}}$ & $5.14_{-0.02}^{+0.02}$ & $4.26_{-0.02}^{+0.02}$ \\
\hline $36^{\mathrm{b}}$ & $221_{-10}^{+10}$ & $56_{-30}^{+15}$ & $5.18_{-0.03}^{+0.03}$ & $5.42_{-0.37}^{+0.11}$ \\
\hline 37 & $2_{-1}^{+33}$ & $5_{-1}^{+1}$ & $2.89_{-1.20}^{+1.20}$ & $4.00_{-0.15}^{+0.16}$ \\
\hline $38^{\mathrm{b}}$ & $191_{-54}^{+26}$ & $1_{-1}^{+1}$ & $4.78_{-0.06}^{+0.06}$ & $3.82_{-0.09}^{+0.06}$ \\
\hline 40 & $295_{-10}^{+21}$ & $96_{-5}^{+5}$ & $5.77_{-0.05}^{+0.05}$ & $6.09_{-0.01}^{+0.01}$ \\
\hline $42^{\mathrm{b}}$ & $202_{-5}^{+5}$ & $25_{-5}^{+5}$ & $5.61_{-0.01}^{+0.01}$ & $5.47_{-0.01}^{+0.00}$ \\
\hline 43 & $822_{-98}^{+5}$ & $96_{-5}^{+5}$ & $6.39_{-0.00}^{+0.01}$ & $6.15_{-0.00}^{+0.01}$ \\
\hline $44^{\mathrm{b}}$ & $111_{-5}^{+5}$ & $7_{-1}^{+1}$ & $5.96_{-0.01}^{+0.01}$ & $5.53_{-0.01}^{+0.01}$ \\
\hline 45 & $398_{-5}^{+5}$ & $80_{-5}^{+5}$ & $6.01_{-0.00}^{+0.00}$ & $6.11_{-0.00}^{+0.00}$ \\
\hline $47^{\mathrm{b}}$ & $100_{-5}^{+6}$ & $6_{-1}^{+1}$ & $5.50_{-0.02}^{+0.02}$ & $\begin{array}{l}5.01_{-0.12}^{+0.02} \\
\end{array}$ \\
\hline \multicolumn{5}{|c|}{$\frac{\text { NGC } 2841 \text { Associations }}{\text { Best Fit with No Dust }}$} \\
\hline & & & & \\
\hline 2 & $473_{-130}^{+70}$ & $90_{-5}^{+5}$ & $5.49_{-0.08}^{+0.08}$ & $5.46_{-0.05}^{+0.04}$ \\
\hline 6 & $111_{-5}^{+\frac{1}{5}}$ & $15_{-5}^{+5}$ & $4.98_{-0.03}^{+0.03}$ & $4.93_{-0.03}^{+0.03}$ \\
\hline 27 & $989_{-5}^{+5}$ & $116_{-5}^{+5}$ & $6.05_{-0.00}^{+0.03}$ & $5.50_{-0.00}^{+0.03}$ \\
\hline 42 & $989_{-5}^{+5} 11$ & $126_{-5}^{+5}$ & $6.51_{-0.01}^{+0.00}$ & $5.91_{-0.00}^{+0.00}$ \\
\hline 50 & $30_{-5}^{+5}$ & $6_{-1}^{+1}$ & $4.43_{-0.02}^{+0.02}$ & $4.49_{-0.02}^{+0.00}$ \\
\hline 55 & $822_{-5}^{+5}$ & $100_{-5}^{+5}$ & $6.28_{-0.00}^{+0.02}$ & $6.00_{-0.00}^{+0.02}$ \\
\hline 61 & $35_{-5}^{+10}$ & $6_{-1}^{+1^{-5}}$ & $4.27_{-0.14}^{+0.14}$ & $4.28_{-0.04}^{+0.03}$ \\
\hline \multicolumn{5}{|c|}{ Best Fit with Dust } \\
\hline $1^{\mathrm{a}}$ & $2_{-1}^{+2}$ & $4_{-1}^{+1}$ & $2.86_{-0.12}^{+0.12}$ & $3.91_{-0.17}^{+0.12}$ \\
\hline 3 & $\begin{array}{c}-1 \\
1000_{-11}^{+>5}\end{array}$ & $\begin{array}{l}-1 \\
191_{-49}^{+16}\end{array}$ & $6.19_{-0.01}^{+0.01}$ & $5.67_{-0.05}^{+0.03}$ \\
\hline $4^{\mathrm{b}}$ & $295_{-44}^{+40}$ & $35_{-5}^{+\overline{5}}$ & $5.15_{-0.11}^{+0.11}$ & $5.09_{-0.04}^{+0.03}$ \\
\hline 5 & $195_{-9}^{+5}$ & $20_{-5}^{+5}$ & $5.32_{-0.02}^{+0.02}$ & $5.16_{-0.02}^{+0.02}$ \\
\hline
\end{tabular}


Table 5-Continued

\begin{tabular}{|c|c|c|c|c|}
\hline \multirow{2}{*}{$\begin{array}{c}\text { Association } \\
\text { ID }\end{array}$} & \multicolumn{2}{|c|}{ Age (Myr) } & \multicolumn{2}{|c|}{$\log \operatorname{Mass}\left(M_{\odot}\right)$} \\
\hline & $E(B-V)=0.0$ & $E(B-V)=0.3$ & $E(B-V)=0.0$ & $E(B-V)=0.3$ \\
\hline $7^{\mathrm{b}}$ & $202_{-5}^{+5}$ & $25_{-5}^{+5}$ & $5.47_{-0.02}^{+0.02}$ & $5.34_{-0.02}^{+0.02}$ \\
\hline 8 & $126_{-5}^{+15}$ & $3_{-1}^{+6}$ & $5.00_{-0.06}^{+0.06}$ & $4.13_{-0.03}^{+0.54}$ \\
\hline $9^{\mathrm{b}}$ & $227_{-5}^{+5}$ & $56_{-30}^{+10}$ & $5.34_{-0.01}^{+0.01}$ & $5.60_{-0.36}^{+0.06}$ \\
\hline $10^{\mathrm{a}}$ & $1_{-1}^{+9}$ & $3_{-2}^{+1}$ & $2.78_{-0.46}^{+0.46}$ & $3.67_{-0.07}^{+0.20}$ \\
\hline 11 & $822_{-130}^{+>178}$ & $111_{-5}^{+5}$ & $6.02_{-0.10}^{+0.10}$ & $5.61_{-0.03}^{+0.08}$ \\
\hline 12 & $295_{-23}^{+36}$ & $100_{-65}^{+5}$ & $5.16_{-0.09}^{+0.09}$ & $5.46_{-0.38}^{+0.06}$ \\
\hline 13 & $\begin{array}{l}725_{-108}^{+>276} \\
-102\end{array}$ & $100_{-5}^{+6}$ & $5.75_{-0.13}^{+0.13}$ & $5.52_{-0.08}^{+0.00}$ \\
\hline 14 & $653_{-51}^{+141}$ & $106_{-6}^{+5}$ & $5.80_{-0.10}^{+0.10}$ & $5.56_{-0.01}^{+0.08}$ \\
\hline 15 & $1000_{-5}^{++>5}$ & $989_{-5}^{+11}$ & $6.81_{-0.00}^{+0.00}$ & $6.82_{-0.00}^{+0.01}$ \\
\hline 16 & $822_{-37}^{+100}$ & $111_{-5}^{+5}$ & $6.18_{-0.05}^{+0.05}$ & $5.74_{-0.01}^{+0.08}$ \\
\hline $17^{\mathrm{b}}$ & $195_{-23}^{+16}$ & $1_{-1}^{+1}$ & $4.93_{-0.04}^{+0.04}$ & $4.00_{-0.08}^{+0.04}$ \\
\hline $18^{\mathrm{b}}$ & $221_{-10}^{+10}$ & $25_{-24}^{+5}$ & $5.04_{-0.04}^{+0.04}$ & $4.95_{-0.86}^{+0.03}$ \\
\hline $19^{\mathrm{b}}$ & $316_{-65}^{+19}$ & $35_{-5}^{+5}$ & $5.25_{-0.06}^{+0.06}$ & $5.14_{-0.02}^{+0.02}$ \\
\hline $20^{\mathrm{b}}$ & $989_{-5}^{+5}$ & $116_{-5}^{+5}$ & $6.51_{-0.00}^{+0.00}$ & $5.97_{-0.00}^{+0.00}$ \\
\hline 21 & $35_{-5}^{+76}$ & $4_{-1}^{+1}$ & $4.27_{-0.54}^{+0.54}$ & $4.08_{-0.04}^{+0.03}$ \\
\hline $22^{\mathrm{b}}$ & $106_{-6}^{+5}$ & $6_{-1}^{+1}$ & $4.97_{-0.03}^{+0.03}$ & $4.50_{-0.12}^{+0.01}$ \\
\hline 23 & $1000_{-67}^{+>5}$ & $166_{-6}^{+16}$ & $6.19_{-0.00}^{+0.00}$ & $5.66_{-0.01}^{+0.03}$ \\
\hline $24^{\mathrm{a}}$ & $2_{-1}^{+2}$ & $5_{-1}^{+1}$ & $2.88_{-0.12}^{+0.12}$ & $3.96_{-0.06}^{+0.15}$ \\
\hline 25 & $1000_{-11}^{+>5}$ & $182_{-16}^{+4}$ & $6.29_{-0.00}^{+0.00}$ & $5.78_{-0.02}^{+0.01}$ \\
\hline 26 & $934_{-295}^{+>67}$ & $106_{-5}^{+5}$ & $\begin{array}{r}-0.00 \\
5.99_{-0.03}^{+0.03}\end{array}$ & $5.58_{-0.08}^{+0.02}$ \\
\hline $28^{\mathrm{b}}$ & $216_{-5}^{+5}$ & $56_{-30}^{+10}$ & $5.20_{-0.02}^{+0.02}$ & $5.44_{-0.36}^{+0.07}$ \\
\hline 29 & $35_{-5}^{+\overline{5}}$ & $5_{-1}^{+1}$ & $4.27_{-0.04}^{+0.04}$ & $4.18_{-0.04}^{+0.04}$ \\
\hline $30^{\mathrm{a}}$ & $35_{-5}^{+5}$ & $5_{-1}^{+1}$ & $4.18_{-0.05}^{+0.05}$ & $4.10_{-0.04}^{+0.04}$ \\
\hline 31 & $120_{-5}^{+10}$ & $8_{-1}^{+1}$ & $5.10_{-0.02}^{+0.02}$ & $\begin{array}{l}4.72_{-0.02}^{+0.02} \\
\end{array}$ \\
\hline 32 & $257_{-20}^{+106}$ & $1_{-1}^{+1}$ & $4.74_{-0.27}^{+0.27}$ & $3.90_{-0.04}^{+0.04}$ \\
\hline 33 & $1000_{-11}^{+>5}$ & $989_{-5}^{+>11}$ & $6.14_{-0.00}^{+0.00}$ & $6.13_{-0.00}^{+0.01}$ \\
\hline 34 & $251_{-9}^{+180}$ & $85_{-5}^{+5}$ & $5.20_{-0.38}^{+0.38}$ & $5.62_{-0.03}^{+0.01}$ \\
\hline 35 & $316_{-65}^{+27}$ & $35_{-5}^{+5}$ & $4.93_{-0.08}^{+0.08}$ & $4.83_{-0.03}^{+0.11}$ \\
\hline 36 & $1000_{-11}^{+>5}$ & $989_{-5}^{+5}>11$ & $5.96_{-0.00}^{+0.08}$ & $5.96_{-0.00}^{+0.03}$ \\
\hline $37^{\mathrm{b}}$ & $313_{-40}^{+22}$ & $35_{-5}^{+5}$ & $5.24_{-0.07}^{+0.07}$ & $5.14_{-0.01}^{+0.02}$ \\
\hline $38^{\mathrm{b}}$ & $111_{-5}^{+5}$ & $7_{-1}^{+8}$ & $4.74_{-0.04}^{+0.04}$ & $4.32_{-0.04}^{+0.41}$ \\
\hline $39^{\mathrm{b}}$ & $251_{-14}^{+112}$ & $30_{-5}^{+20}$ & $4.90_{-0.26}^{+0.04}$ & $4.89_{-0.03}^{+0.04}$ \\
\hline $40^{\mathrm{b}}$ & $237_{-16}^{+143}$ & $30_{-5}^{+35}$ & $4.84_{-0.28}^{+0.28}$ & $4.80_{-0.05}^{+0.39}$ \\
\hline 41 & $1000_{-5}^{+>5}$ & $126_{-5}^{+5}$ & $6.51_{-0.00}^{+0.00}$ & $5.92_{-0.00}^{+0.00}$ \\
\hline 43 & $1000_{-5}^{+>5}$ & $989_{-5}^{+5}$ & $6.59_{-0.00}^{+0.00}$ & $6.59_{-0.00}^{+0.00}$ \\
\hline 44 & $822_{-146}^{+>178}$ & $100_{-5}^{+5}$ & $5.82_{-0.07}^{+0.07}$ & $5.53_{-0.01}^{+0.04}$ \\
\hline 45 & $1000_{-56}^{+>5}$ & $116_{-5}^{+4}$ & $6.11_{-0.01}^{+0.01}$ & $5.56_{-0.02}^{+0.04}$ \\
\hline 46 & $1000_{-11}^{+>5}$ & $141_{-5}^{+5}$ & $6.40_{-0.01}^{+0.01}$ & $5.83_{-0.01}^{+0.00}$ \\
\hline 47 & $35_{-5}^{+5}$ & $5_{-1}^{+2}$ & $3.98_{-0.09}^{+0.01}$ & $3.90_{-0.07}^{+0.16}$ \\
\hline 48 & $1000_{-5}^{+>5}$ & $989_{-5}^{+5}$ & $6.65_{-0.00}^{+0.00}$ & $6.65_{-0.00}^{+0.00}$ \\
\hline 49 & $989_{-5}^{+>11}$ & $111_{-5}^{+5}$ & $6.60_{-0.01}^{+0.01}$ & $6.10_{-0.00}^{+0.00}$ \\
\hline $51^{\mathrm{b}}$ & $126_{-6}^{+5}$ & $8_{-1}^{+1}$ & $5.04_{-0.04}^{+0.01}$ & $4.66_{-0.02}^{+0.02}$ \\
\hline 52 & $1000_{-45}^{+}>5$ & $120_{-4}^{+10}$ & $6.03_{-0.02}^{+0.04}$ & $5.50_{-0.05}^{+0.03}$ \\
\hline $53^{\mathrm{b}}$ & $227_{-10}^{+5}$ & $25_{-5}^{+5}$ & $5.09_{-0.03}^{+0.03}$ & $5.00_{-0.02}^{+0.02}$ \\
\hline $54^{\mathrm{b}}$ & $216_{-5}^{+5}$ & $56_{-30}^{+10}$ & $5.25_{-0.02}^{+0.02}$ & $5.48_{-0.36}^{+0.07}$ \\
\hline 56 & $1000_{-34}^{+>5}$ & $120_{-4}^{+16}$ & $6.04_{-0.01}^{+0.01}$ & $5.49_{-0.05}^{+0.02}$ \\
\hline 57 & $295_{-50}^{+52}$ & $35_{-5}^{+\overline{5}^{4}}$ & $4.99_{-0.14}^{-0.01}$ & $4.92_{-0.03}^{-0.05}$ \\
\hline $58^{\mathrm{b}}$ & $246_{-8}^{+15}$ & $40_{-5}^{+45}$ & $5.26_{-0.02}^{+0.02}$ & $5.33_{-0.10}^{+0.35}$ \\
\hline 59 & $653_{-51}^{+169}$ & $100_{-5}^{+6}$ & $5.69_{-0.12}^{+0.12}$ & $5.52_{-0.08}^{+0.01}$ \\
\hline $60^{\mathrm{b}}$ & $202_{-11}^{+10}$ & $1_{-1}^{+1^{-5}}$ & $5.00_{-0.03}^{+0.12}$ & $4.08_{-0.02}^{+0.02}$ \\
\hline $62^{\mathrm{b}}$ & $211_{-10}^{-1 / 1}$ & $20_{-5}^{-15}$ & $5.19_{-0.02}^{+0.03}$ & $5.06_{-0.02}^{+0.02}$ \\
\hline $63^{\mathrm{b}}$ & $202_{-5}^{+5}$ & $20_{-5}^{+5}$ & $5.19_{-0.02}^{+0.02}$ & $5.04_{-0.02}^{+0.02}$ \\
\hline $64^{\mathrm{b}}$ & $211_{-5}^{+10}$ & $1_{-1}^{+1}$ & $5.00_{-0.02}^{+0.02}$ & $4.06_{-0.03}^{+0.03}$ \\
\hline 65 & $1000_{-5}^{+>5}$ & $207_{-16}^{+20}$ & $6.27_{-0.01}^{+0.01}$ & $5.71_{-0.06}^{+0.03}$ \\
\hline 66 & $2_{-1}^{+8}$ & $5_{-1}^{+1}$ & $2.68_{-0.52}^{+0.52}$ & $3.77_{-0.10}^{+0.16}$ \\
\hline 67 & $1000_{-11}^{+>5}$ & $126_{-5}^{+5}$ & $6.27_{-0.00}^{+0.00}$ & $5.67_{-0.00}^{+0.00}$ \\
\hline 68 & $934_{-62}^{+>67}$ & $111_{-5}^{+5}$ & $6.28_{-0.03}^{+0.03}$ & $5.80_{-0.01}^{+0.01}$ \\
\hline
\end{tabular}


Table 5-Continued

\begin{tabular}{|c|c|c|c|c|}
\hline \multirow{2}{*}{$\begin{array}{c}\text { Association } \\
\text { ID }\end{array}$} & \multicolumn{2}{|c|}{ Age (Myr) } & \multicolumn{2}{|c|}{$\log \operatorname{Mass}\left(M_{\odot}\right)$} \\
\hline & $E(B-V)=0.0$ & $E(B-V)=0.3$ & $E(B-V)=0.0$ & $E(B-V)=0.3$ \\
\hline 69 & $2+33$ & $5_{-1}^{+1}$ & $2.82^{+1.19}$ & $3.91_{-0.15}^{+0.15}$ \\
\hline $70^{\mathrm{b}}$ & $216^{+50}$ & $1^{-1}$ & $4.61^{-1}+0.08$ & $3.72^{-0.06}$ \\
\hline 71 & $638_{-43}^{+284}$ & $106_{-5}^{+5}$ & $5.59_{-0.20}^{+0.20}$ & $5.40_{-0.10}^{+0.04}$ \\
\hline 72 & $1000_{-5}^{+43}$ & $989_{-5}^{+5}>11$ & $6.65_{-0.00}^{+0.20}$ & $6.65_{-0.00}^{+0.01}$ \\
\hline 73 & $1000_{-5}^{+>5}$ & $313_{-11}^{+5}$ & $6.74_{-0.00}^{+0.00}$ & $6.15_{-0.02}^{+0.00}$ \\
\hline $74^{\mathrm{b}}$ & $221_{-10}^{+\overline{5}}$ & $56_{-5}^{+15}$ & $5.20_{-0.03}^{+0.03}$ & $5.45_{-0.02}^{+0.11}$ \\
\hline 75 & $1000_{-5}^{+>5}$ & $989_{-5}^{+}>11$ & $6.68_{-0.00}^{+0.00}$ & $6.68_{-0.00}^{+0.01}$ \\
\hline \multicolumn{5}{|c|}{$\frac{\text { NGC } 3621 \text { Associations }}{\text { Best Fit with No Dust }}$} \\
\hline 1 & $6^{+1}$ & $6^{+1}$ & $3.26^{+0.02}$ & $3.87^{+0.00}$ \\
\hline 2 & $130_{-4}^{+6}$ & $9_{-1}^{-1}$ & $4.89_{-0.02}^{+0.02}$ & $4.47_{-0.02}^{+0.02}$ \\
\hline 3 & $100_{-5}^{+5}$ & $6_{-1}^{-1}$ & $4.92_{-0.01}^{+0.02}$ & $4.39^{+0.02}$ \\
\hline 4 & $120^{-5}+10$ & $2^{-1}$ & $5.16^{+0.01}$ & $4.30^{+0.01}$ \\
\hline $5^{\mathrm{a}}$ & $40_{-15}^{+\overline{5} 0}$ & $6_{-1}^{+1}$ & $4.34_{-0.40}^{+0.40}$ & $4.27_{-0.13}^{+0.03}$ \\
\hline 6 & $100_{-5}^{+5}$ & $6_{-1}^{-1}$ & $5.43_{-0.01}^{+0.40}$ & $4.93_{-0.01}^{+0.13}$ \\
\hline 8 & $232_{-5}^{+11}$ & $80_{-10}^{+1}$ & $4.76_{-0.02}^{+0.01}$ & $5.15_{-0.04}^{+0.01}$ \\
\hline 10 & $106_{-5}^{+5}$ & $7_{-1}^{+1}$ & $5.18_{-0.01}^{+0.01}$ & $4.71_{-0.01}^{+0.01}$ \\
\hline 11 & $85_{-5}^{+\overline{5}}$ & $6_{-1}^{+1}$ & $4.78_{-0.04}^{+0.01}$ & $4.33_{-0.02}^{+0.01}$ \\
\hline $14^{\mathrm{a}}$ & $\begin{array}{l}-5 \\
100_{-10}^{+6}\end{array}$ & $6_{-1}^{-1}$ & $\begin{array}{l}-0.04 \\
4.37^{+0.04}\end{array}$ & $3.86_{-0.03}^{+0.02}$ \\
\hline 15 & $90^{+\overline{5}^{-10}}$ & $6^{-1}$ & $5.17^{-0.04}$ & $4.69^{-0.12}$ \\
\hline 16 & $80_{-15}^{+5}$ & $6_{-1}^{-1}$ & $4.51_{-0.05}^{-0.01}$ & $4.08_{-0.03}^{+0.00}$ \\
\hline 18 & $100_{-5}^{+5}$ & $6_{-1}^{+1}$ & $5.4_{-0.01}^{+0.01}$ & $4.96_{-0.00}^{+0.03}$ \\
\hline 20 & $989_{-11}^{+>11}$ & $116_{-5}^{+5}$ & $5.76_{-0.01}^{+0.01}$ & $5.22_{-0.00}^{+0.03}$ \\
\hline 21 & $166_{-50}^{+36}$ & $20_{-12}^{+\overline{5}}$ & $4.37_{-0.12}^{+0.12}$ & $4.26_{-0.49}^{+0.06}$ \\
\hline $23^{\mathrm{a}}$ & $191_{-39}^{+16}$ & $20_{-5}^{+\frac{1}{5}}$ & $4.64_{-0.05}^{+0.05}$ & $4.44_{-0.05}^{+0.19}$ \\
\hline 24 & $130_{-14}^{+59}$ & $15_{-5}^{-5}$ & $4.68_{-0.20}^{+0.20}$ & $4.52_{-0.03}^{+0.03}$ \\
\hline 27 & $106_{-6}^{-\frac{1}{5}}$ & $5_{-1}^{+1^{5}}$ & $4.53_{-0.03}^{+0.20}$ & $3.91_{-0.02}^{+0.13}$ \\
\hline 29 & $85_{-5}^{+\overline{5}^{\circ}}$ & $6_{-1}^{+1}$ & $5.01_{-0.01}^{+0.01}$ & $4.54_{-0.00}^{+0.00}$ \\
\hline 30 & $725_{-101}^{+198}$ & $111_{-5}^{+5}$ & $5.45_{-0.10}^{+0.01}$ & $5.07_{-0.04}^{+0.00}$ \\
\hline 32 & $191_{-5}^{+5}$ & $20_{-5}^{+5^{5}}$ & $5.19_{-0.02}^{+0.10}$ & $5.01_{-0.01}^{+0.04}$ \\
\hline 33 & $243_{-5}^{+5}$ & $85_{-5}^{+5}$ & $5.5_{-0.01}^{+0.01}$ & $5.92_{-0.01}^{+0.01}$ \\
\hline 36 & $106_{-5}^{+5}$ & $6_{-1}^{+1}$ & $4.7_{-0.02}^{+0.01}$ & $4.16^{+0.13}$ \\
\hline 40 & $30_{-5}^{+35}$ & $6_{-1}^{-1}$ & $4.00_{-0.38}^{+0.02}$ & $4.08_{-0.03}^{+0.02}$ \\
\hline 41 & $40^{-55}$ & $6_{-1}^{-1}$ & $4.05^{+0.38}$ & $3.99^{+0.03}$ \\
\hline 42 & $\begin{array}{l}-10 \\
191_{-5}^{+5}\end{array}$ & $20_{-5}^{+1}$ & $5.52_{-0.01}^{-0.43}$ & $5.35_{-0.01}^{-0.12}$ \\
\hline 43 & $80_{-35}^{+15}$ & $6_{-1}^{+\overline{1}^{-5}}$ & $\begin{array}{l}{ }^{-0.01} \\
4.53_{-0.10}^{+0.10}\end{array}$ & $4.14_{-0.02}^{+0.02}$ \\
\hline 44 & $106_{-5}^{+5}$ & $7_{-1}^{+1}$ & $4.88_{-0.02}^{+0.102}$ & $4.44_{-0.02}^{+0.02}$ \\
\hline 45 & $100_{-5}^{+5}$ & $6_{-1}^{+1}$ & $4.66_{-0.02}^{+0.02}$ & $4.15^{+0.02}$ \\
\hline $47^{\mathrm{a}}$ & $106_{-10}^{+5}$ & $6_{-1}^{+1}$ & $4.57_{-0.03}^{+0.03}$ & $4.04_{-0.12}^{+0.13}$ \\
\hline 51 & $85_{-14}^{+10}$ & $6_{-1}^{+1}$ & $4.41_{-0.07}^{+0.07}$ & $3.96_{-0.03}^{+0.03}$ \\
\hline 53 & $\begin{array}{c}-14 \\
251_{-9}^{+161}\end{array}$ & $90_{-5}^{-1}$ & $\begin{array}{r}-0.07 \\
5.13^{+0.36}\end{array}$ & $\begin{array}{r}-0.03 \\
5.53^{+0.02}\end{array}$ \\
\hline 54 & $151^{-9}+35$ & $10^{-5}$ & $4.31^{-0.36}$ & $3.84^{-0.02}$ \\
\hline 57 & $130_{-10}^{+55}$ & $8_{-1}^{+\overline{7}^{1}}$ & $5.25_{-0.01}^{+0.11}$ & $4.86_{-0.00}^{+0.34}$ \\
\hline \multicolumn{5}{|c|}{ Best Fit with Dust } \\
\hline 7 & $725_{-33}^{+5}$ & $106_{-5}^{+5}$ & $6.21_{-0.00}^{+0.00}$ & $5.90_{-0.00}^{+0.00}$ \\
\hline 9 & $582_{-26}^{+14}$ & $100_{-5}^{+5}$ & $5.72_{-0.01}^{+0.00}$ & $5.57_{-0.01}^{+0.00}$ \\
\hline 12 & $785_{-27}^{+37}$ & $106_{-5}^{+5}$ & $5.99_{-0.02}^{+0.01}$ & $5.65_{-0.01}^{+0.01}$ \\
\hline $13^{\mathrm{b}}$ & $221^{-27}$ & $56^{+\overline{5}^{5}}$ & $5.08^{+0.02}$ & $5.33^{+0.01}$ \\
\hline 17 & $1000_{-5}^{+}>5$ & $141_{-5}^{+5}$ & $6.48_{-0.00}^{+0.01}$ & $5.92_{-0.00}^{+0.01}$ \\
\hline 19 & $1000_{-11}^{+5}$ & $141_{-5}^{+5}$ & $5.87_{-0.01}^{+0.00}$ & $5.31_{-0.01}^{+0.00}$ \\
\hline $22^{\mathrm{b}}$ & $227^{+11}$ & $30^{+-56}$ & $4.74^{-0.01}$ & $4.68^{+0.01}$ \\
\hline 25 & $302_{-51}^{+45}$ & $90_{-5}^{+5}$ & $5.00_{-0.11}^{+0.02}$ & $5.32_{-0.03}^{+0.02}$ \\
\hline 26 & $316_{-31}^{+5}$ & $96_{-5}^{+5}$ & $5.14_{-0.00}^{+0.00}$ & $5.41_{-0.00}^{+0.00}$ \\
\hline
\end{tabular}




\section{Table 5-Continued}

\begin{tabular}{|c|c|c|c|c|}
\hline \multirow{2}{*}{$\begin{array}{c}\text { Association } \\
\text { ID }\end{array}$} & \multicolumn{2}{|c|}{ Age $(\mathrm{Myr})$} & \multicolumn{2}{|c|}{$\log$ Mass $\left(M_{\odot}\right)$} \\
\hline & $E(B-V)=0.0$ & $E(B-V)=0.3$ & $E(B-V)=0.0$ & $E(B-V)=0.3$ \\
\hline 28 & $251_{-6}^{+147}$ & $90_{-5}^{+5}$ & $5.03_{-0.33}^{+0.33}$ & $5.44_{-0.00}^{+0.02}$ \\
\hline $31^{\mathrm{b}}$ & $221^{-6}$ & $60^{-5}$ & $5.33+0.013$ & $\begin{array}{r}5.61+0.00 \\
5.04\end{array}$ \\
\hline 34 & $295^{+40}$ & $100^{+5}$ & $4.94^{+0.01}$ & $5.26^{+0.04}$ \\
\hline 35 & $1000^{+29}>5$ & $160^{+42}$ & $5.57^{+0.01}$ & $5.03^{+0.04}$ \\
\hline 37 & $251^{+108}$ & $90^{+5}$ & $4.99^{+0.24}$ & $5.40^{+0.00}$ \\
\hline 38 & $295+40$ & $96^{+5}$ & $4.94^{+0.10}$ & $5.26^{+0.01}$ \\
\hline 39 & $302_{-45}^{+45}$ & $90_{-55}^{+5}$ & $4.81_{-0.12}^{+0.12}$ & $5.14_{-0.02}^{+0.01}$ \\
\hline 46 & $191_{-19}^{+11}$ & $1_{-1}^{+1}$ & $4.67_{-0.03}^{+0.03}$ & $3.68_{-0.03}^{+0.03}$ \\
\hline $48^{\mathrm{b}}$ & $216_{-5}^{+10}$ & $56_{-30}^{+15}$ & $4.80_{-0.03}^{+0.03}$ & $5.05_{-0.39}^{+0.12}$ \\
\hline 49 & $35_{-34}^{+10}$ & $5_{-1}^{+2}$ & $3.78_{-0.14}^{+0.14}$ & $3.68_{-0.05}^{+0.24}$ \\
\hline 50 & $232_{-10}^{+5}$ & $76_{-26}^{+5}$ & $4.92_{-0.02}^{+0.02}$ & $5.31_{-0.21}^{+0.03}$ \\
\hline 52 & $603_{-14}^{+58}$ & $100_{-5}^{+6}$ & $5.29_{-0.05}^{+0.05}$ & $5.16_{-0.07}^{+0.02}$ \\
\hline 55 & $725_{-115}^{+98}$ & $106_{-5}^{+5}$ & $5.44_{-0.06}^{+0.06}$ & $5.13_{-0.08}^{+0.00}$ \\
\hline 56 & $725_{-41}^{+17}$ & $100_{-5}^{+5}$ & $5.78_{-0.01}^{+0.01}$ & $5.54_{-0.00}^{+0.00}$ \\
\hline $58^{\mathrm{b}}$ & $111_{-5}^{+5}$ & $7_{-1}^{+1}$ & $5.05_{-0.00}^{+0.00}$ & $4.61_{-0.01}^{+0.01}$ \\
\hline \multicolumn{5}{|c|}{$\frac{\text { NGC 5055 Associations }}{\text { Best Fit with No Dust }}$} \\
\hline 12 & $989_{-5}^{+5}$ & $96_{-5}^{+5}$ & $6.11_{-0.00}^{+0.00}$ & $5.80_{-0.00}^{+0.00}$ \\
\hline \multicolumn{5}{|c|}{ Best Fit with Dust } \\
\hline 1 & $1000_{-5}^{+>5}$ & $126_{-5}^{+5}$ & $6.79_{-0.00}^{+0.00}$ & $6.19_{-0.00}^{+0.00}$ \\
\hline 2 & $316_{-59}^{+11}$ & $96_{-5}^{+5}$ & $5.23_{-0.03}^{+0.03}$ & $5.50_{-0.01}^{+0.04}$ \\
\hline 3 & $1000_{-5}^{+>5}$ & $251_{-19}^{+15}$ & $6.50_{-0.00}^{+0.00}$ & $5.83_{-0.00}^{+0.04}$ \\
\hline 4 & $1000_{-11}^{+>5}$ & $116_{-5}^{+4}$ & $6.35_{-0.00}^{+0.00}$ & $5.81_{-0.01}^{+0.00}$ \\
\hline 5 & $989_{-11}^{+>11}$ & $111_{-5}^{+5}$ & $6.00_{-0.01}^{+0.01}$ & $5.50_{-0.04}^{+0.00}$ \\
\hline 6 & $216_{-14}^{+15}$ & $25_{-24}^{+5}$ & $4.81_{-0.04}^{+0.04}$ & $4.72_{-0.88}^{+0.04}$ \\
\hline 7 & $1000_{-11}^{+>5}$ & $126_{-5}^{+5}$ & $6.28_{-0.00}^{+0.00}$ & $5.69_{-0.00}^{+0.00}$ \\
\hline 8 & $989_{-5}^{+>11}$ & $126_{-10}^{+5}$ & $6.11_{-0.01}^{+0.01}$ & $5.52_{-0.00}^{+0.05}$ \\
\hline 9 & $1000_{-11}^{+>5}$ & $126_{-5}^{+5}$ & $6.32_{-0.01}^{+0.01}$ & $5.73_{-0.00}^{+0.00}$ \\
\hline 10 & $989_{-5}^{+>11}$ & $111_{-5}^{+5}$ & $6.11_{-0.01}^{+0.01}$ & $5.60_{-0.00}^{+0.00}$ \\
\hline 11 & $1000_{-11}^{+}>5$ & $120_{-5}^{+5}$ & $6.58_{-0.00}^{+0.00}$ & $6.03_{-0.00}^{+0.00}$ \\
\hline 13 & $989_{-11}^{+>11}$ & $116_{-5}^{-5}$ & $6.11_{-0.01}^{+0.01}$ & $5.57_{-0.02}^{+0.00}$ \\
\hline 14 & $35_{-34}^{+\overline{8} 5}$ & $4_{-1}^{+1 \overline{1}}$ & $\begin{array}{r}3.75_{-0.60}^{+0.60} \\
\end{array}$ & $3.57_{-0.18}^{+0.74}$ \\
\hline 15 & $1000_{-11}^{+>5}$ & $126_{-5}^{+5}$ & $6.13_{-0.00}^{+0.00}$ & $5.54_{-0.01}^{+0.00}$ \\
\hline 16 & $243_{-21}^{+133}$ & $30_{-5}^{+20}$ & $4.72_{-0.29}^{+0.29}$ & $4.69_{-0.26}^{+0.26}$ \\
\hline 17 & $603_{-20}^{+130}$ & $100_{-5}^{+5}$ & $5.55_{-0.11}^{+0.11}$ & $5.42_{-0.02}^{+0.02}$ \\
\hline
\end{tabular}

${ }^{a}$ These associations are missing their $4.5 \mu \mathrm{m}$ point and are thus only fit with three datapoints.

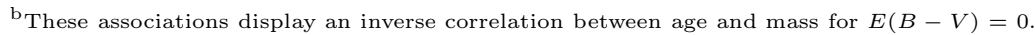




\section{Results}

\subsection{Dust vs. No Dust}

Our results are presented below for each galaxy in three categories: 'no dust' $(E(B-V)$ $=0)$, 'dust' $(E(B-V)=0.3)$, and 'best fit' (either dust or no dust). We use two criteria to determine the 'best fit' category.

1. As stated above, there exists a positive correlation between the age and mass of an association. In $47(\sim 21 \%)$ cases, however, we see an inverse correlation in the confidence intervals of cases involving no dust. Figure 9 shows a typical example. All of these cases become positively correlated when dust is added to the system and, for $\sim 68 \%$ of these cases, adding dust results in a lower minimum $\chi^{2}$ value by $\sim 49 \%$ on average. Finally, $\sim 83 \%$ of these cases show excess 8.0 micron emission, indicating PAH emission in the vicinity of these associations. Therefore, we conclude that these regions are good candidates for higher relative extinction and that the inverse correlation is an artifact of improperly treating the extinction in the no dust case.
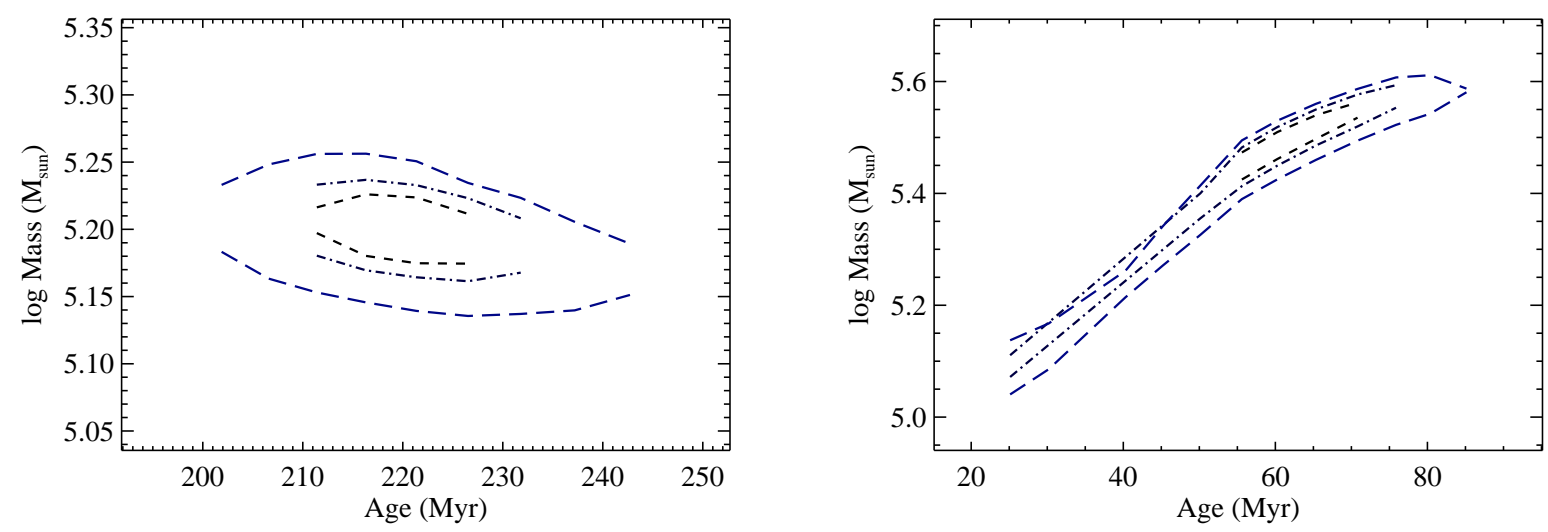

Fig. 9.- Association 74 from NGC 2841 displays a typical inverse correlation (left) in the confidence intervals between age and mass. When dust is added to the models, association 74 presents the expected positive correlation between age and mass (right).

2. For all remaining associations in which the positive correlation between age and mass appears for both the no dust and dust cases, the best fit is determined by the reduced $\chi^{2}$ value. $\sim 34 \%$ of associations have a lower reduced $\chi^{2}$ with no added dust and $\sim 45 \%$ of associations have a lower $\chi^{2}$ value when modeled with dust $(E(B-V)=0.3)$. However, it should be noted that both fits are often acceptable (i.e. within the $1 \sigma$ errors) and so we report both solutions while counting these associations as dusty for 
the 'best fit' statistics. The distribution of minimum $\chi^{2}$ between the dust and no dust cases will be discussed on a galaxy-by-galaxy basis below.

Associations are further divided into three categories: ionizing $(<10 \mathrm{Myr})$, non-ionizing (10 Myr-1 Gyr), and evolved ( $>1$ Gyr). Since our criteria for removing contaminating foreground stars (e.g. Equation (1)) and background galaxies also deplete our sample of associations older than $\sim 1$ Gyr, our statistics for such associations would be biased and we will not discuss the evolved associations any further.

All $\chi^{2}$ values shown or discussed are reduced with $\mathrm{N}=3$ and minimum $\chi^{2}$ comparisons are based on median values.

\subsection{NGC 0628}

The imaged region of NGC 0628 contains 32 star forming associations. A dust map of this field (Figure 10 (left)) is consistent with low extinction in excess of the foreground as almost none of our stellar complexes are associated with dust emission. Table 6 shows the break-down of associations into types, the average and median age and mass of all associations, and the age dispersion. The latter spans the entire range of our models when no dust is applied. When the most extreme dust case is applied to all associations, the age range is still large enough to account for a lack of $\mathrm{H} \alpha$ relative to UV. Figure 11 shows the age and mass distribution. Figure 12 shows the relative $\chi^{2}$ distribution association by association comparing the no dust case to the dust case. The no dust case results in lower minimum $\chi^{2}$ values by $8 \%$. 7 associations $(\sim 22 \%)$ show an inverse correlation between age and mass with no dust, with $5(\sim 16 \%)$ showing both an inverse correlation and a better $\chi^{2}$ with dust. $8(\sim 25 \%)$ additional associations have a better $\chi^{2}$ with dust. These numbers are reflected in the best fit values. 

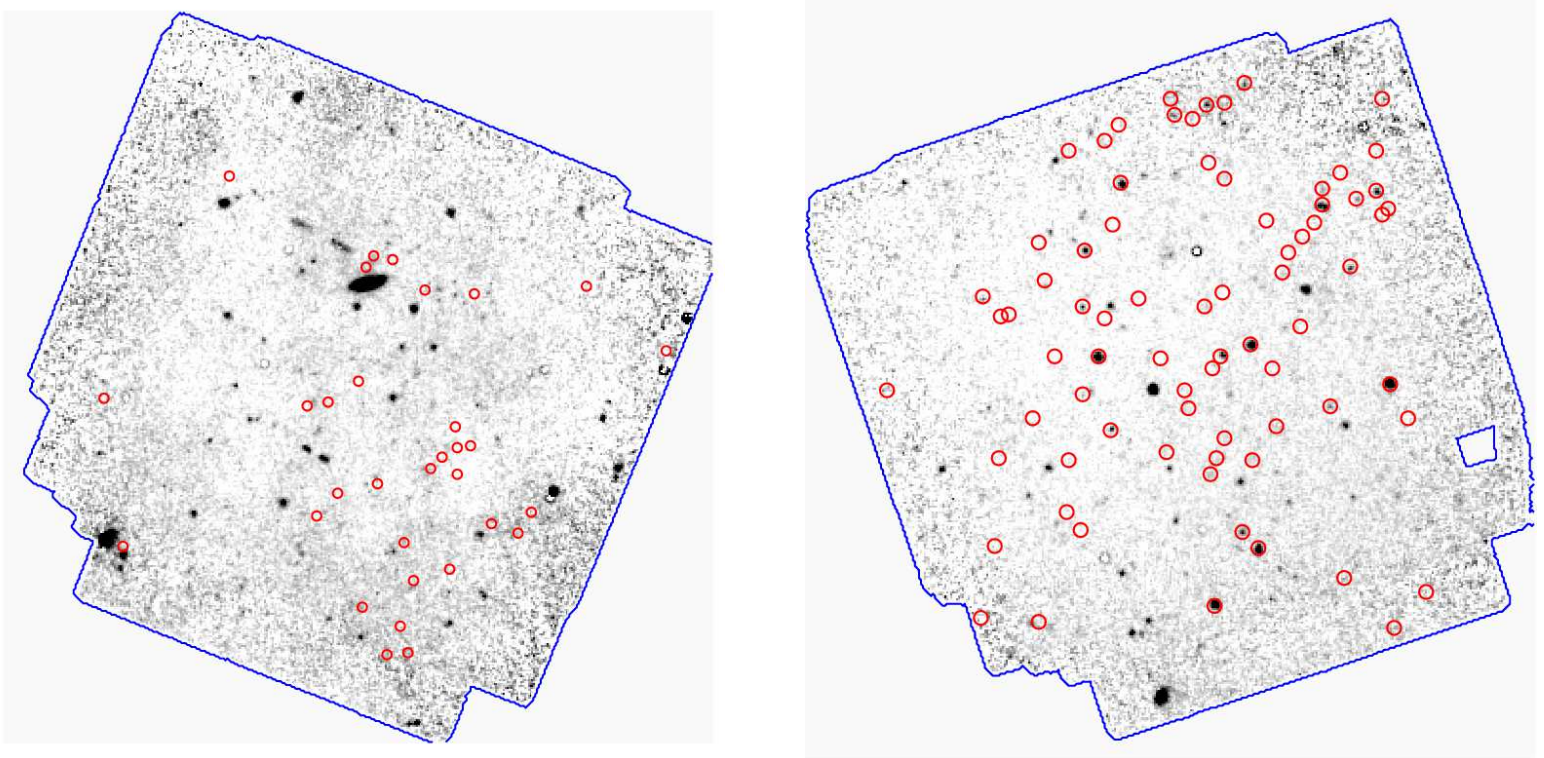

Fig. 10.- The dust maps of NGC 0628 (left) and NGC 2841 (right) were created by scaling the $3.6 \mu \mathrm{m}$ image to approximate the continuum emission and then subtracting from the $8.0 \mu \mathrm{m}$ image. Bright sources are indicative of dust excess as traced by the $8.0 \mu \mathrm{m}(\mathrm{PAH}$ emission), though these sources may be background galaxies due to their intrinsic dust emission. The red apertures indicate UV bright stellar complexes. It is clear that not many UV bright regions are associated with dust, though there are more in NGC 2841, which is supported by overall better $\chi^{2}$ values found when dust extinction is applied to the observed SEDs (Section 5.4). It should be noted that the source of the dust emission and the source of the UV emission are not necessarily associated. 

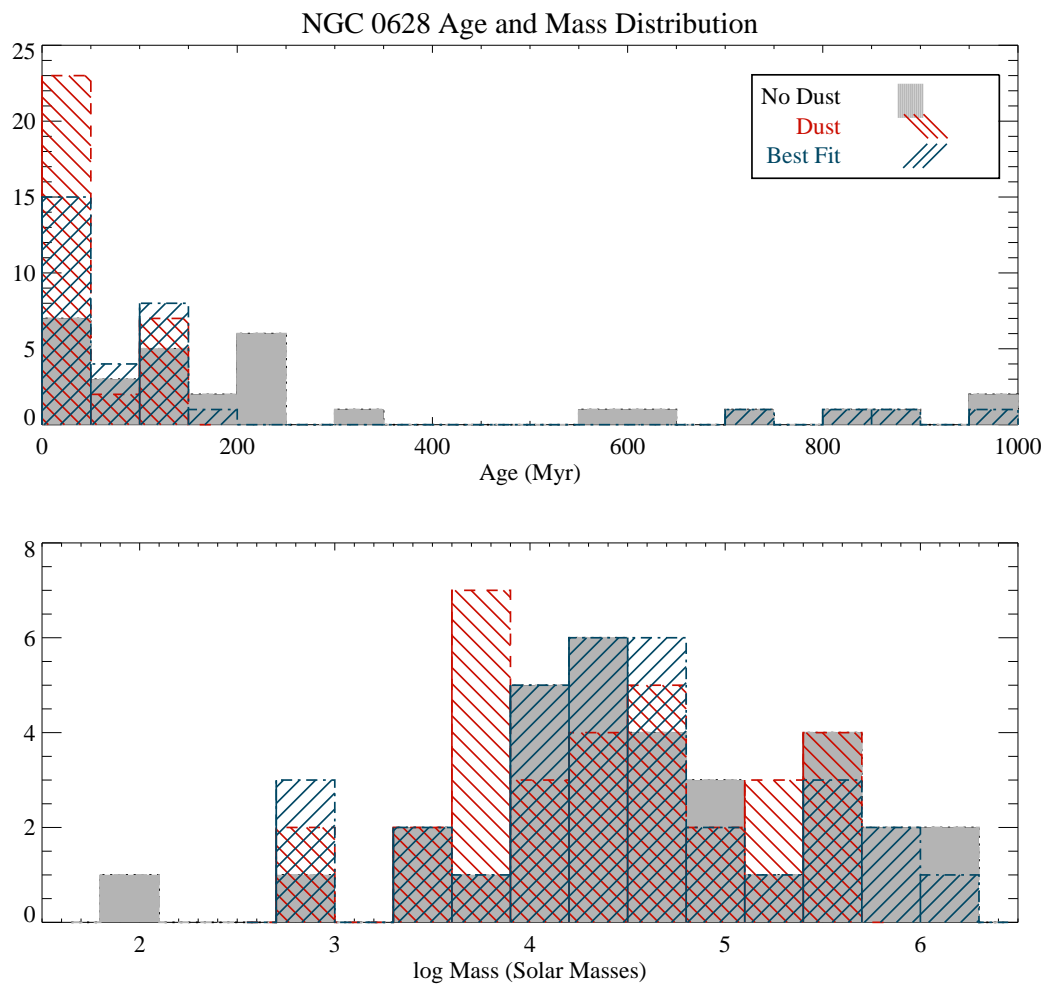

Fig. 11. - The age and mass distribution for NGC 0628. The no dust $E(B-V)=0.0$, dust $E(B-V)=0.3$, and the 'best fit' case are shown, as indicated in the legend. 

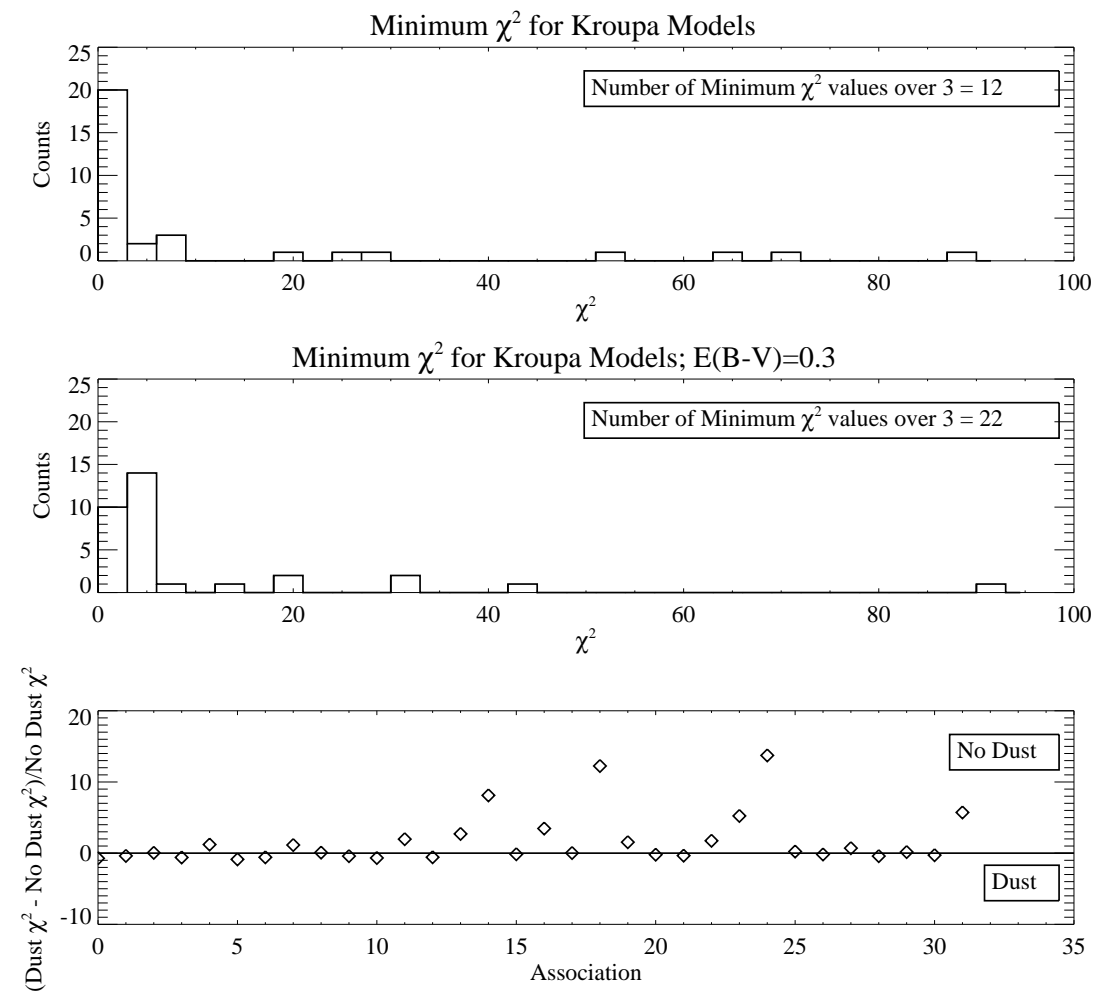

Fig. 12.- NGC 0628 - The $\chi^{2}$ distribution demonstrates that overall this galaxy has a better fit without dust (top) than with it (middle). Combined with the dust map and the number of occurrences of inverse correlation plots, this leads us to conclude that this is a relatively low-dust galaxy. The bottom panel shows the relative degree of improvement in the $\chi^{2}$ value for the no dust or dust cases. When analysed by association a few sources indicates a much better fit with dust. Further studies are needed to ascertain the true nature of the dust on these individual scales.

With the best fit values for age, the percentage of ionizing associations is $\sim 18 \%$, which is consistent with $\mathrm{H} \alpha$ observations in outer disks.

Table 6. NGC 0628 Association Statistics

\begin{tabular}{ccccccc}
\hline \hline & $\begin{array}{c}\text { Ionizing } \\
(<10 \mathrm{Myr})\end{array}$ & $\begin{array}{c}\text { Non-Ionizing } \\
(10 \mathrm{Myr}-1 \mathrm{Gyr})\end{array}$ & $\begin{array}{c}\text { Evolved } \\
(>1 \mathrm{Gyr})\end{array}$ & $\begin{array}{c}\text { Average (Median) } \\
\text { Age }[\mathrm{Myr}]\end{array}$ & $\begin{array}{c}\text { log Average (Median) } \\
\text { Mass }\left[M_{\odot}\right]\end{array}$ & $\begin{array}{c}\text { Age } \\
\text { Range }[\mathrm{Myr}]\end{array}$ \\
\hline No Dust & $2(\sim 6 \%)$ & $29(\sim 90 \%)$ & $1(\sim 3 \%)$ & $297(191)$ & $4.69(4.57)$ & $1-1000$ \\
Dust & $16(\sim 50 \%)$ & $16(\sim 50 \%)$ & 0 & $37(15)$ & $4.39(4.39)$ & $1-125$ \\
Best Fit & $6(\sim 18 \%)$ & $26(\sim 81 \%)$ & 0 & $158(60)$ & $4.48(4.48)$ & $1-988$ \\
\hline
\end{tabular}




\section{3. $\quad$ NGC 2090}

The portion of NGC 2090 imaged contains 47 star forming associations. Table 7 shows the break-down of associations by type, the average and median age and mass of all associations, and the age dispersion. Its dust map (not shown) shows about half of its stellar complexes are not associated with any dust, while the other half, hugging close to the spiral arms, may be. However, the percentage of associations with inverse correlations or better $\chi^{2}$ fits with dust is similar to that of NGC 0628 and these may just be chance superpositions. Figure 13 shows the age and mass distributions and Figure 14 shows the $\chi^{2}$ distribution. The no dust case has lower minimum $\chi^{2}$ by $64 \%$ on average. 12 associations ( $\left.26 \%\right)$ show an inverse correlation between age and mass with no dust, with $5(\sim 11 \%)$ showing both an inverse correlation and a better $\chi^{2}$ with dust. $13(\sim 28 \%)$ associations have a better $\chi^{2}$ with dust. $8(\sim 13 \%)$ associations are ionizing.
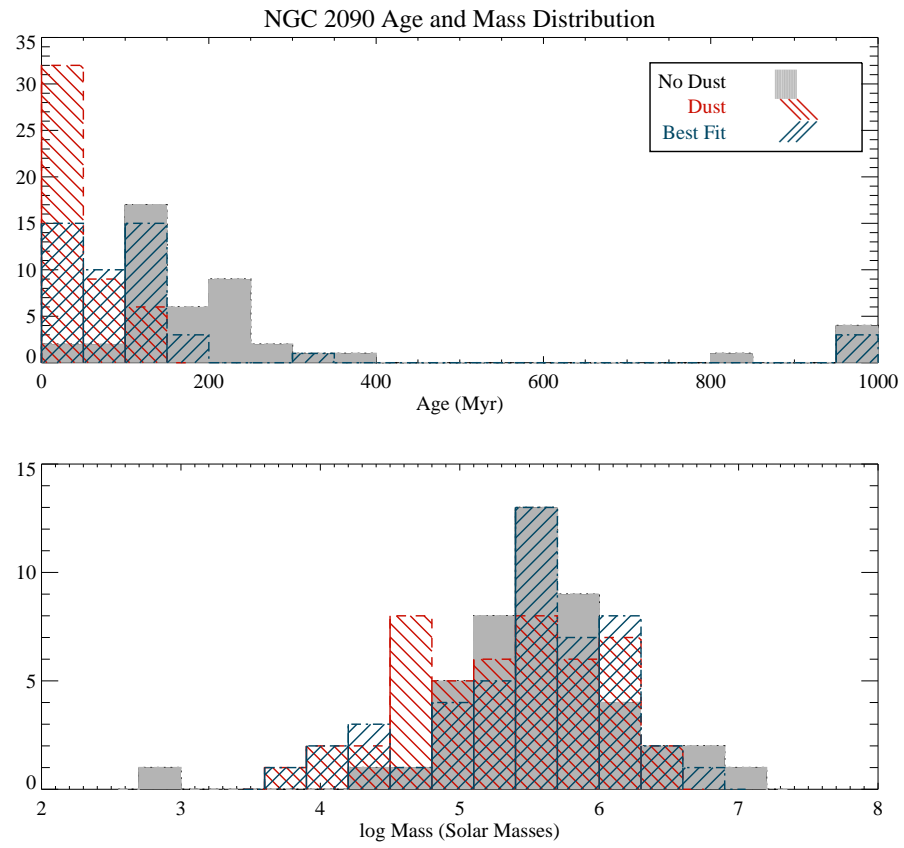

Fig. 13.- The age and mass distribution for NGC 2090, with conventions as in Figure 11. 

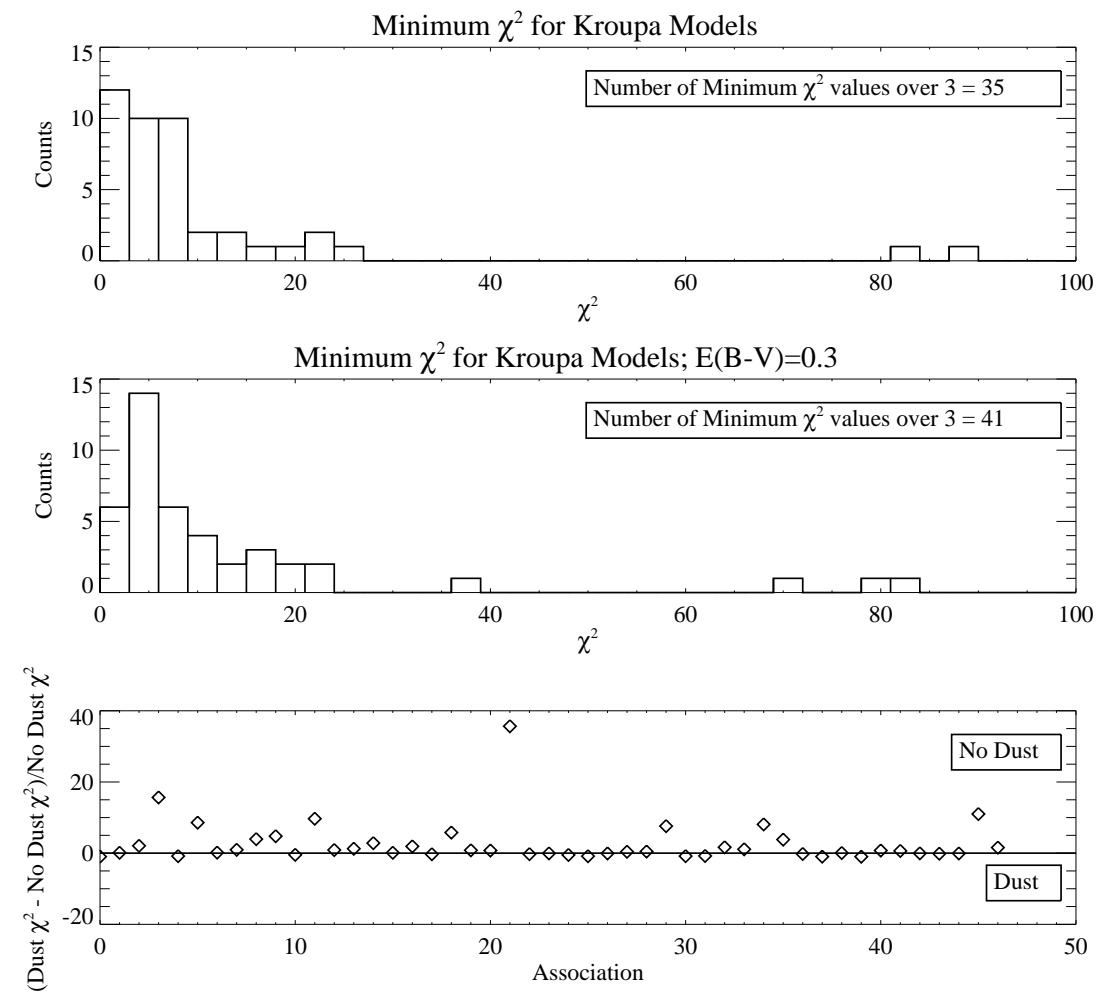

Fig. 14. - NGC 2090 - As with NGC 0628, from a straight analysis of the minimum $\chi^{2}$ values, this galaxy appears to have low dust content except for a few associations.

\subsection{NGC 2841}

NGC 2841 has 75 associations in the portion imaged by IRAC and is by far the most likely candidate of our galaxy sample to have relatively large amounts of dust. 23 ( 31\%) associations show an inverse correlation between age and mass with no dust. 19 of those also show a decrease in the minimum $\chi^{2}$ (Figure 16) and an additional $45(\sim 60 \%)$ associations show this same decrease with a positive correlation. The associations that show some sign

Table 7. NGC 2090 Association Statistics

\begin{tabular}{ccccccc}
\hline \hline & $\begin{array}{c}\text { Ionizing } \\
(<10 \mathrm{Myr})\end{array}$ & $\begin{array}{c}\text { Non-Ionizing } \\
(10 \mathrm{Myr}-1 \mathrm{Gyr})\end{array}$ & $\begin{array}{c}\text { Evolved } \\
(>1 \mathrm{Gyr})\end{array}$ & $\begin{array}{c}\text { Average (Median) } \\
\text { Age [Myr] }\end{array}$ & $\begin{array}{c}\text { log Average (Median) } \\
\text { Mass }\left[M_{\odot}\right]\end{array}$ & $\begin{array}{c}\text { Age } \\
\text { Range [Myr] }\end{array}$ \\
\hline No Dust & $1(\sim 2 \%)$ & $44(\sim 93 \%)$ & $2(\sim 4 \%)$ & $283(191)$ & $5.57(5.62)$ & $2-1000$ \\
Dust & $23(\sim 48 \%)$ & $24(\sim 51 \%)$ & 0 & $38(15)$ & $5.29(5.39)$ & $1-216$ \\
Best Fit & $8(\sim 17 \%)$ & $39(\sim 82 \%)$ & 0 & $141(96)$ & $5.48(5.47)$ & $1-988$ \\
\hline
\end{tabular}


of dust also have excess $8.0 \mu \mathrm{m}$ emission in all cases except five, where the $8.0 \mu \mathrm{m}$ point is missing. The dust map (Figure 10 (right)) shows quite a few complexes very obviously associated with dust emission and the minimum $\chi^{2}$ value improves with dust by $36 \%$ on average.

Due to the relative importance of dust in this galaxy, we questioned (as mentioned above) whether a different dust model may yield a significant change in our results. So far we have assumed a simple model of Milky Way-type dust extinction which places all of the dust in front of the star forming region. To test this, we also apply a dust attenuation model (Calzetti et al. 2000), which places the dust within the region, and find ages intermediate between the no dust and dust extinction case. However, we also find that the overall fits are $\sim 30 \%$ worse in the dust attenuation case as measured by the minimum $\chi^{2}$ value.

Table 8 shows the break-down of the associations. The average age with dust is $\sim 150$ Myr, which is in good agreement with the average ages from the previous two galaxies and the median age of associations in M83 (Dong et al. 2008). This age doesn't change much when the best fits are taken into account. The age range, however, still spans our models. The age and mass distributions can be seen in Figure [15, $19(\sim 25 \%)$ associations are ionizing.
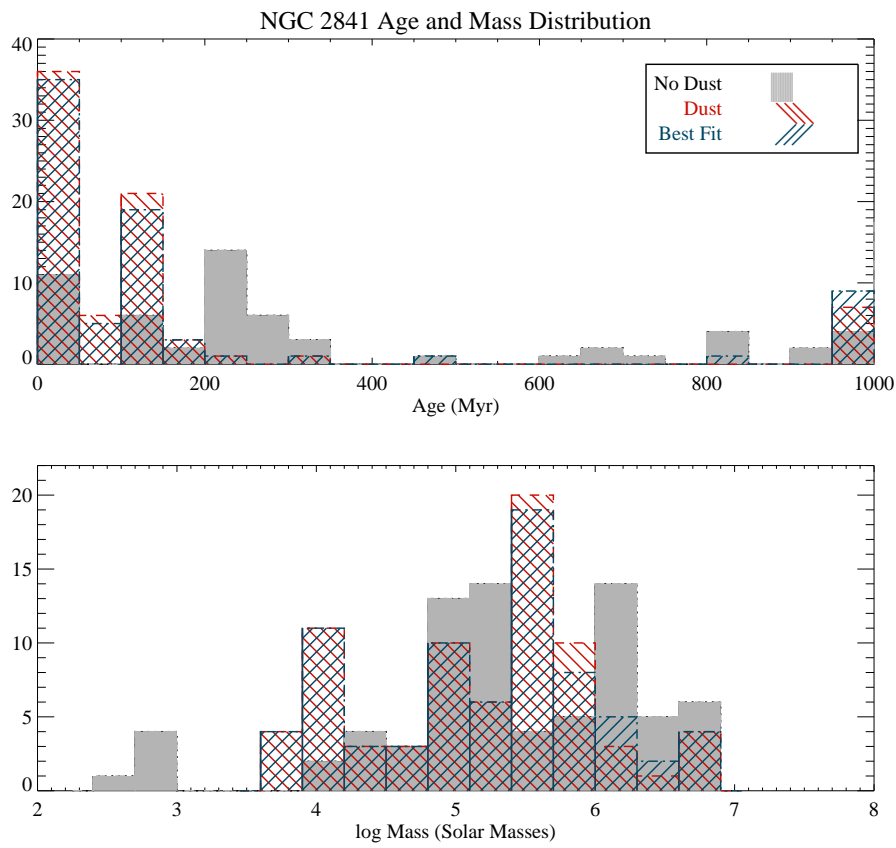

Fig. 15.- As in Figure 11, the ages and mass distribution for NGC 2841. 

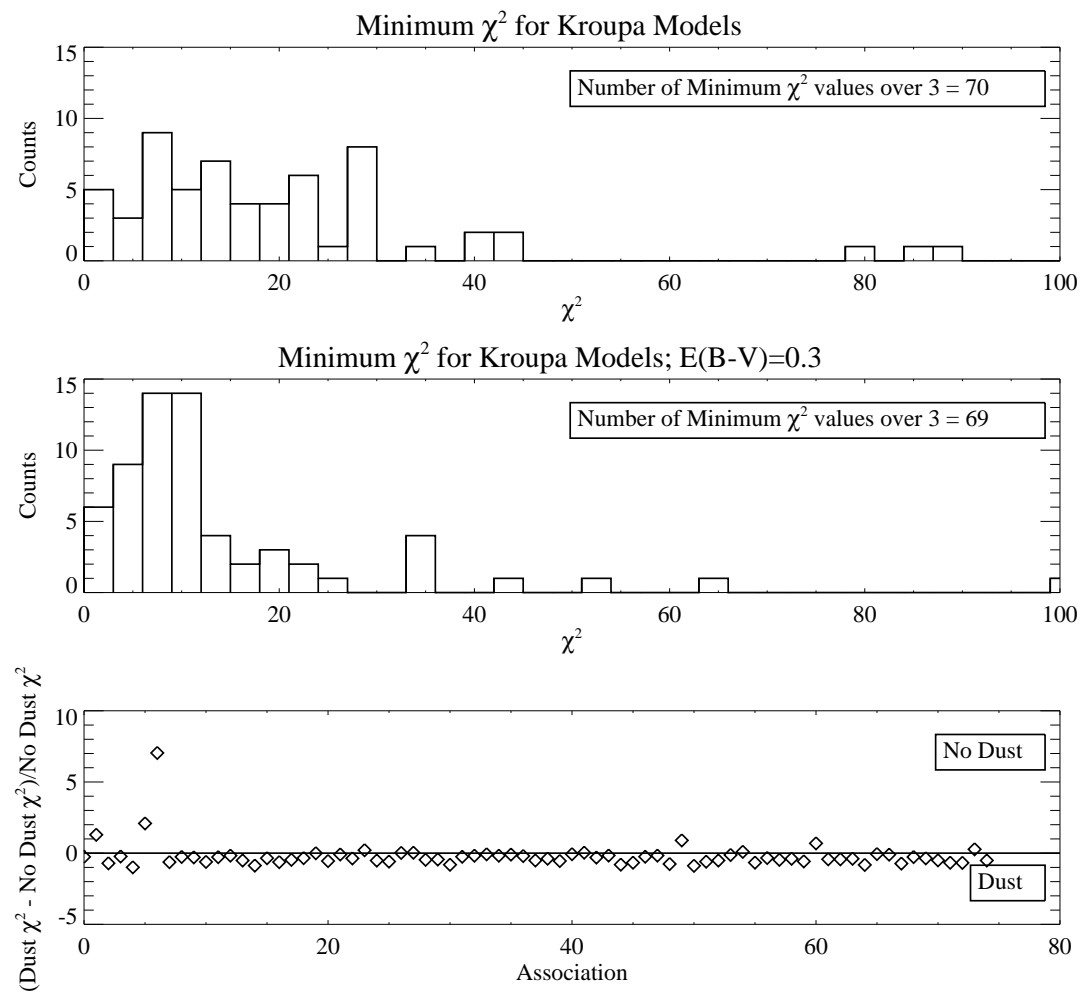

Fig. 16. - NGC 2841 - Associations in NGC 2841 show better fits in the case of $E(B-V)$ $=0.3$, both in the overall minimum $\chi^{2}$ values (top, middle) and in the strengths of the improvement for individual associations (bottom). This is further supported by the number of inverse correlations between age and mass in the no dust case.

Table 8. NGC 2841 Association Statistics

\begin{tabular}{ccccccc}
\hline \hline & $\begin{array}{c}\text { Ionizing } \\
(<10 \mathrm{Myr})\end{array}$ & $\begin{array}{c}\text { Non-Ionizing } \\
(10 \mathrm{Myr}-1 \mathrm{Gyr})\end{array}$ & $\begin{array}{c}\text { Evolved } \\
(>1 \mathrm{Gyr})\end{array}$ & $\begin{array}{c}\text { Average (Median) } \\
\text { Age }[\mathrm{Myr}]\end{array}$ & $\begin{array}{c}\text { log Average (Median) } \\
\text { Mass }\left[M_{\odot}\right]\end{array}$ & $\begin{array}{c}\text { Age } \\
\text { Range }[\mathrm{Myr}]\end{array}$ \\
\hline No Dust & $5(\sim 6 \%)$ & $52(\sim 70 \%)$ & $18(\sim 24 \%)$ & $496(295)$ & $5.36(5.25)$ & $1-1000$ \\
Dust & $21(\sim 28 \%)$ & $54(\sim 72 \%)$ & 0 & $150(56)$ & $5.18(5.44)$ & $1-998$ \\
Best Fit & $19(\sim 25 \%)$ & $56(\sim 74 \%)$ & 0 & $190(56)$ & $5.20(5.44)$ & $1-988$ \\
\hline
\end{tabular}




\subsection{NGC 3621}

NGC 3621 contains 58 associations in the IRAC FOV and is our closest galaxy and deepest imaging. It displays very little signs of dust, with only $5(\sim 9 \%)$ associations with a inverse correlation between age and mass and $20(\sim 34 \%)$ associations where dust improves the minimum $\chi^{2}$. Table 9 shows the association break-down and the $\chi^{2}$ improvement is $174 \%$ in the no dust case. The age and mass distributions can be seen in Figure 17 and $\chi^{2}$ distribution in Figure 18, $4(\sim 6 \%)$ associations are ionizing.
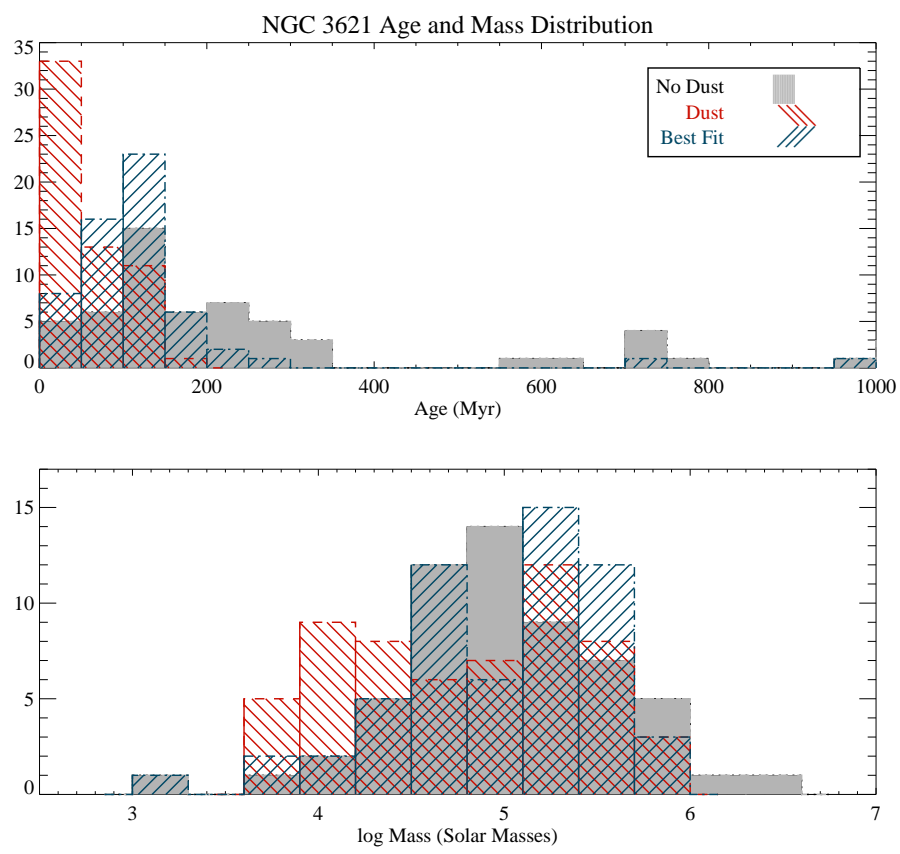

Fig. 17.- As in Figure 11, the ages and mass distribution for NGC 3621. 

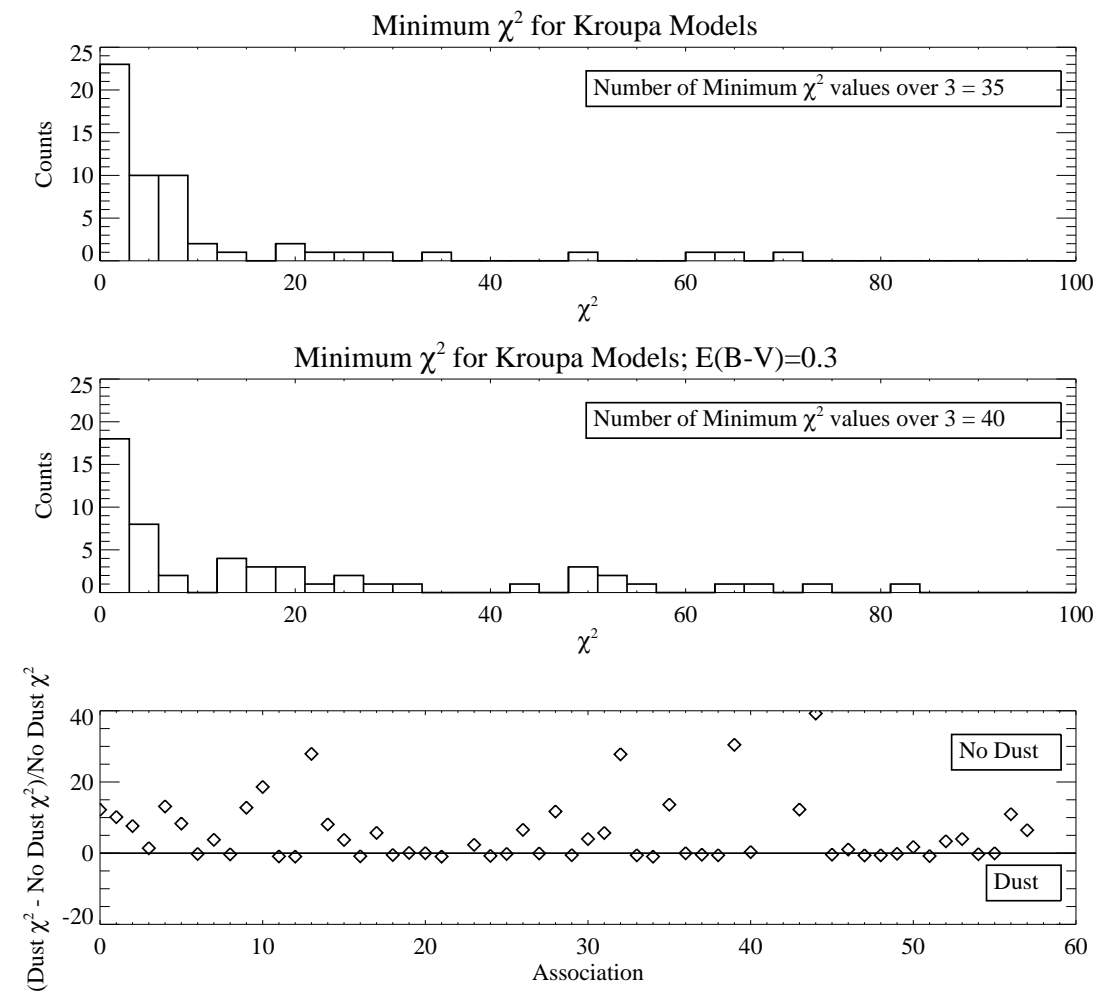

Fig. 18. - NGC 3621 - Like NGC 0628 and NGC 2090, the $\chi^{2}$ distribution for NGC 3621 does not indicate a strong dust presence.

Table 9. NGC 3621 Association Statistics

\begin{tabular}{ccccccc}
\hline \hline & $\begin{array}{c}\text { Ionizing } \\
(<10 \mathrm{Myr})\end{array}$ & $\begin{array}{c}\text { Non-Ionizing } \\
(10 \mathrm{Myr}-1 \mathrm{Gyr})\end{array}$ & $\begin{array}{c}\text { Evolved } \\
(>1 \mathrm{Gyr})\end{array}$ & $\begin{array}{c}\text { Average (Median) } \\
\text { Age [Myr] }\end{array}$ & $\begin{array}{c}\text { log Average (Median) } \\
\text { Mass }\left[M_{\odot}\right]\end{array}$ & $\begin{array}{c}\text { Age } \\
\text { Range [Myr] }\end{array}$ \\
\hline No Dust & $1(\sim 2 \%)$ & $54(\sim 93 \%)$ & $3(\sim 5 \%)$ & $278(191)$ & $4.98(4.99)$ & $6-1000$ \\
Dust & $27(\sim 46 \%)$ & $31(\sim 53 \%)$ & 0 & $47(20)$ & $4.79(4.93)$ & $1-160$ \\
Best Fit & $4(\sim 7 \%)$ & $54(\sim 93 \%)$ & 0 & $129(100)$ & $4.96(5.13)$ & $1-988$ \\
\hline
\end{tabular}




\subsection{NGC 5055}

While 146 associations were detected in the region imaged in NGC 5055, the FUV and NUV images are intrinsically faint and contain high levels of background noise spikes, which SExtractor had difficulty separating from actual sources. As such, noise spikes had to be eliminated by hand before removing foreground stars and background galaxies. The final conservative association count is 17 , with fully half of those classified as evolved without dust. As can be seen in Table 10, the average ages in all three categories is higher than for previous galaxies. The age and mass distributions can be seen in Figure 19 and the $\chi^{2}$ distribution in Figure 20, with a $\chi^{2}$ improvement in the dust case of $43 \%$. Only $1(\sim 6 \%)$ association is ionizing.
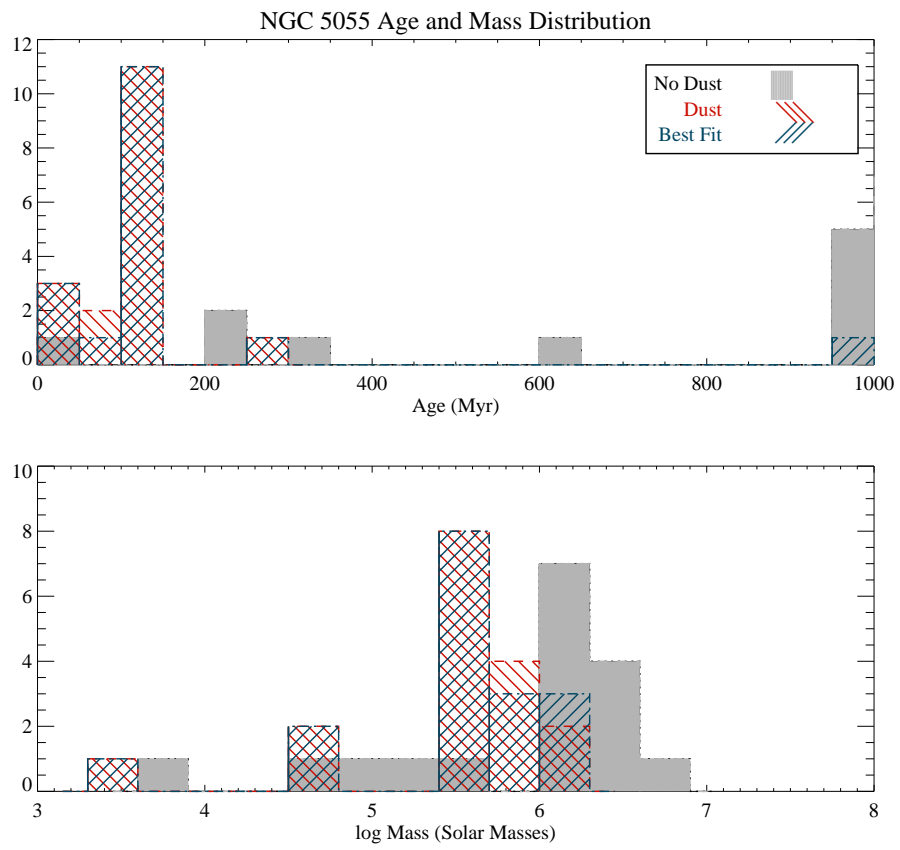

Fig. 19.- As in Figure 11, the ages and mass distribution for NGC 5055. 

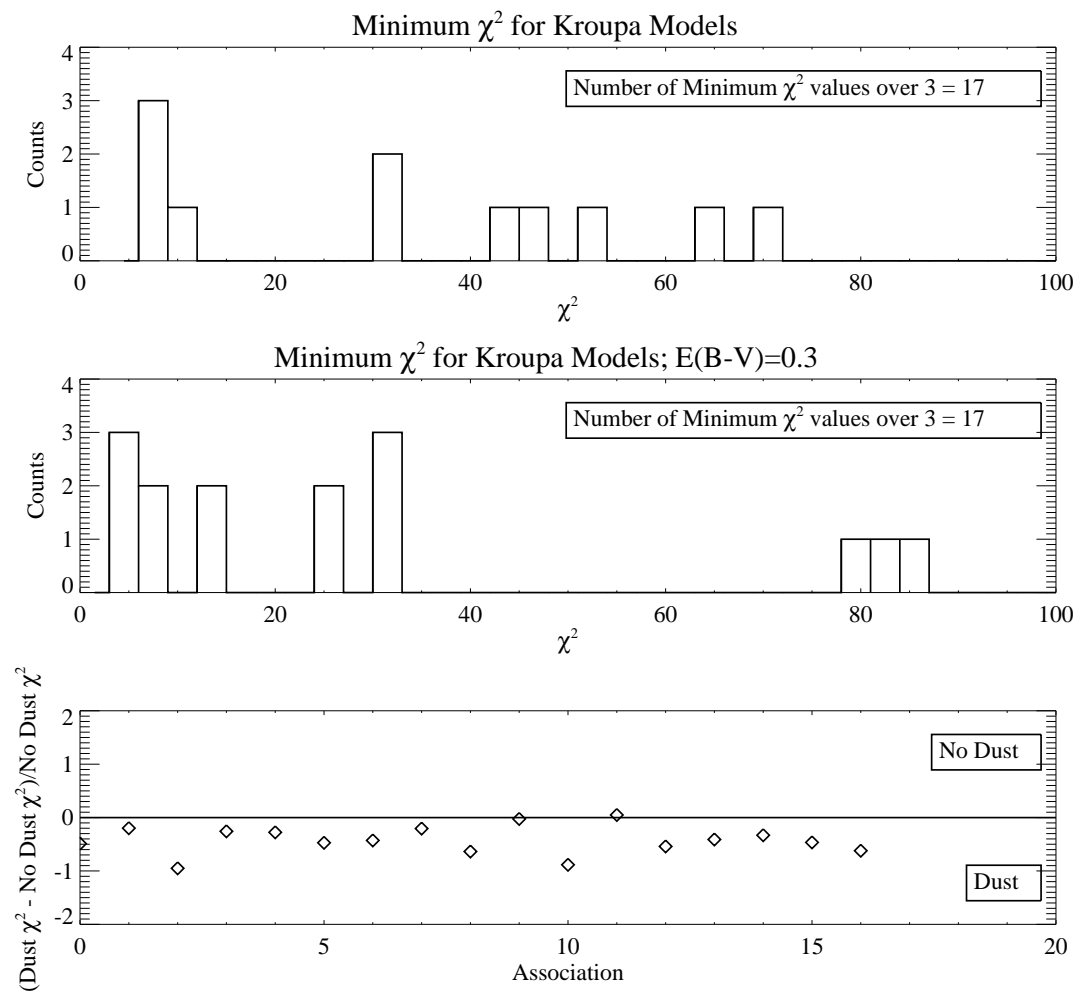

Fig. 20. - NGC 5055 - While the same evidence that led us to suppose that the outer regions of NGC 2841 are relatively dusty compared to our other galaxies is present here, it is hard to tell due to the small number of associations and the large amount of noise in the NGC 5055 UV images.

\subsection{Cumulative Properties}

The cumulative statistics of these five galaxies have demonstrated that star forming associations in the outer disks are represented by a large age dispersion with a median age of $\sim 100 \mathrm{Myr}$ and an average age of $\sim 158 \mathrm{Myr}$ (Table 11). Though extinction has

Table 10. NGC 5055 Association Statistics

\begin{tabular}{ccccccc}
\hline \hline & $\begin{array}{c}\text { Ionizing } \\
(<10 \mathrm{Myr})\end{array}$ & $\begin{array}{c}\text { Non-Ionizing } \\
(10 \mathrm{Myr}-1 \mathrm{Gyr})\end{array}$ & $\begin{array}{c}\text { Evolved } \\
(>1 \mathrm{Gyr})\end{array}$ & $\begin{array}{c}\text { Average (Median) } \\
\text { Age [Myr] }\end{array}$ & $\begin{array}{c}\text { log Average (Median) } \\
\text { Mass }\left[M_{\odot}\right]\end{array}$ & $\begin{array}{c}\text { Age } \\
\text { Range }[\mathrm{Myr}]\end{array}$ \\
\hline No Dust & 0 & $10(\sim 58 \%)$ & $7(\sim 41 \%)$ & $786(989)$ & $5.85(6.11)$ & $35-1000$ \\
Dust & $1(\sim 6 \%)$ & $16(\sim 94 \%)$ & 0 & $106(116)$ & $5.45(5.72)$ & $4-998$ \\
Best Fit & $1(\sim 6 \%)$ & $16(\sim 94 \%)$ & 0 & $159(116)$ & $5.47(5.57)$ & $4-988$ \\
\hline
\end{tabular}


a non-negligible effect on the age determinations of any individual association, the large percentage of young associations that are nevertheless beyond the ionizing stage within the extinction range assumed here is consistent with the presence of UV emission and the scarcity of $\mathrm{H} \alpha$ emission, (Thilker et al. 2005; Gil de Paz et al. 2005; Goddard et al. 2010). These associations also show results consistent with low dust and low extinction in outer disk, although, again, more in-depth studies are needed to determine the dust content of individual associations.

The selection effects due to the detections limits in all bands can be seen in Figure 21 . The dominant effect is that we begin to lose sensitivity to associations below $\sim 10^{4} M_{\odot}$ for ages older than $\sim 50$ Myr due to the FUV sensitivity. In addition, $\sim 25 \%$ of our associations fall into low signal to noise regions in the IRAC coverage maps, which affects our completeness as we may not have selected very young (blue) associations in these regions with high UV flux but undetected $3.6 \mu \mathrm{m}$ emission.

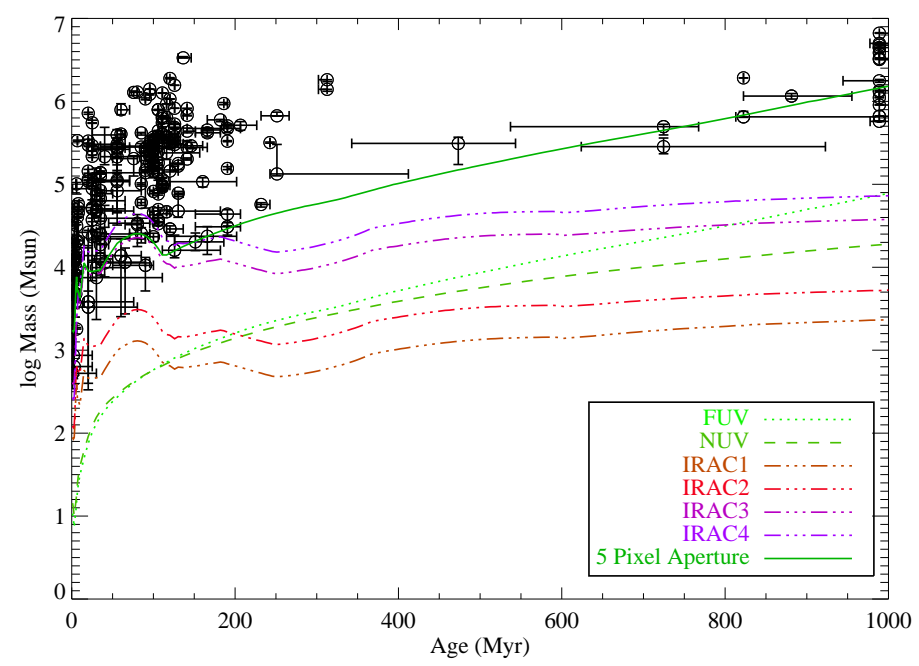

Fig. 21. - Logarithmic mass $\left(M_{\odot}\right)$ as a function of the age (Myr) of all star forming associations. The dotted and dashed lines indicate our per pixel detection limits, as determined by the $2 \sigma$ sensitivity in each band. The limiting sensitivity for a 5 pixel aperture is also indicated. For ages $\lesssim 50 \mathrm{Myr}$, the minimum detectable cluster mass is set by the $3.6 \mu \mathrm{m}$ sensitivity; for ages $\gtrsim 50 \mathrm{Myr}$, the limit is set by the FUV.

The age and masses of all associations in this study can be seen in Figure 22, which demonstrates the wide range of ages seen in the star forming associations in outer disks. Unfortunately, the incompleteness of our sample means that the two most prominent features in this histogram - the peak at $\sim 100 \mathrm{Myr}$ and the paucity of associations beyond $\sim 200 \mathrm{Myr}$ - are best explained as selection effects due to the fact that sources were originally selected to 
be FUV bright. As the FUV mainly traces the youngest stages of SF, this selects heavily for ages $<100$ Myr and heavily against associations $>200$ Myr. A simple Poissonian analysis of the 100 Myr peak shows it to be at the $6 \sigma$ level, indicating that in the age range to which we are most sensitive star forming associations are preferentially on the older end. However, studies have shown that age determinations based on integrated colors can result in age pile-ups (Hunter et al. 2003; Fall et al. 2005). A more complete sample is needed before any determination about the star formation history of outer disks can be made. It is also important to note that since our models stop at $1 \mathrm{Gyr}$, associations that are listed as 1 Gyr actually have undetermined ages and, in the 'best fit' category, they are preferentially selected as dusty, due to the fact that the presence of dust yields younger ages and we lack old-age (red) models without dust. Finally, none of our galaxies are undergoing a major merger, but there is evidence for interactions with small companions which may provide regular triggering of star formation.
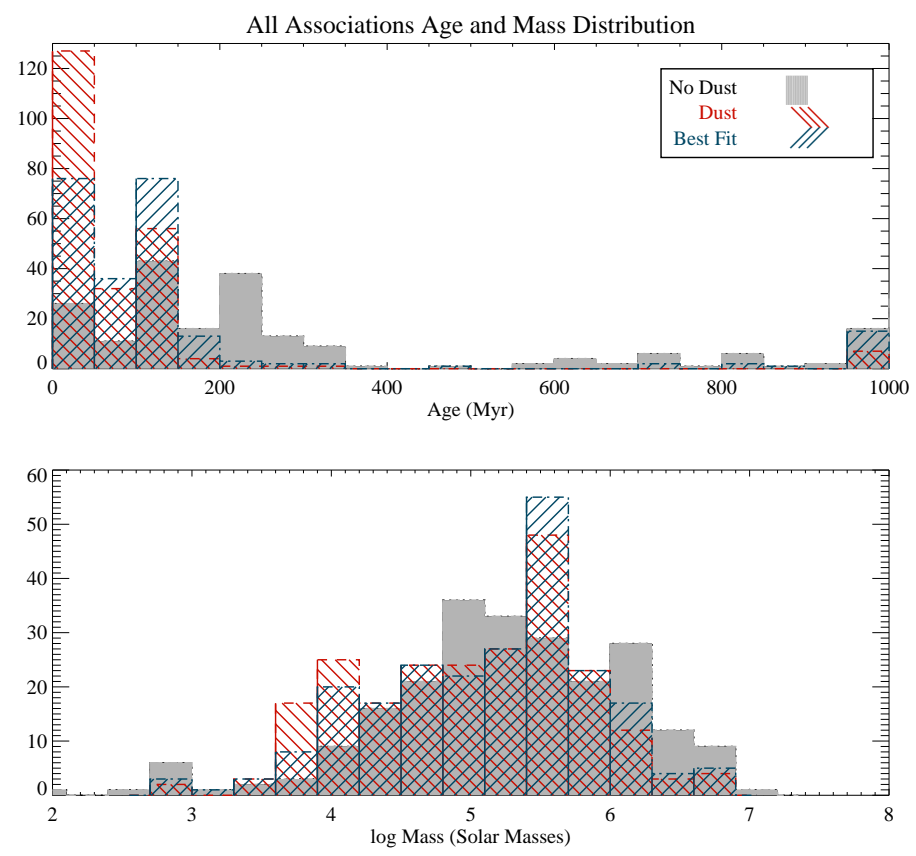

Fig. 22. - This histogram includes all associations. From this we can see that the median age is $\sim 100$ Myr and the range of ages is populated. Two things should be noted here: 1) Associations beyond $\sim 200$ Myr are underrepresented due to selection bias. 2) Associations categorized as 1 Gyr have undetermined ages due to the range of our models (1 Myr-1 Gyr). In addition, due to the lack of models at older ages, these associations are represented in the 'best fit' category as dusty associations as dust reddens the SEDs and provides a better fit to old (red) associations. 


\subsection{Comparison with $\mathrm{H} \alpha$ Observations}

Though most of the galaxies in this study have been imaged in $\mathrm{H} \alpha$ as part of the Spitzer Infrared Nearby Galaxies Survey (SINGS; Kennicutt (2003)), only NGC 3621 has observations extending to the outer disk regions. We performed a simple spatial comparison to determine if our stellar clusters are associated with $\mathrm{H} \alpha$ emission. 39 (66\%) of the associations have no $\mathrm{H} \alpha$ detections while the other 19 (34\%) are spatially coincident with $\mathrm{H} \alpha$ detections ranging from strong point sources to nearby diffuse emission. Associations that have observed $\mathrm{H} \alpha$ emission are generally younger with a median age for $E(B-V)=0.0$ $(E(B-V)=0.3)$ of 100 (6) Myr for those with, and 231 (80) Myr for those without. Due to the large uncertainties in our age determinations, this is consistent with our results. It is also important to note due to our selection criteria (using the FUV to select stellar clusters and then requiring IRAC data and other criteria to reduce contaminants), we do not claim a complete sample and thus cannot make a fair comparison with the number of $\mathrm{H} \alpha$ sources. A statistical sample of $\mathrm{H} \alpha$ emitting sources across several XUV disks would allow us to extend this study and further evaluate the nature of outer disk star-forming clusters.

\section{IMF Analysis and Discussion}

In previous sections, we determined the age and masses of star forming association in outer disks for two extreme values of extinction with a fixed Kroupa IMF and found that at both extremes, there exists a wide age dispersion with a median age older than that at which ionizing stars will still exist. We now address how a difference in the IMF will affect our age determinations.

There are two possible modifications to the IMF that could be explored that would be consistent with the observed lack of $\mathrm{H} \alpha$ : either the IMF can be truncated at some value, suggesting that there is some mechanism preventing high mass stars from forming (Pflamm-Altenburg et al. 2009; Weidner \& Kroupa 2004, 2005), or the slope of the IMF can be steepened. Due to the coarse timesteps of our models and the assumed ages of our associations (beyond the ionizing stage), we believe that we are not sensitive to the differences

Table 11. Total Association Statistics

\begin{tabular}{ccc}
\hline \hline & $\begin{array}{c}\text { Average (Median) } \\
\text { Age }[\mathrm{Myr}]\end{array}$ & $\begin{array}{c}\text { log Average (Median) } \\
\text { Mass }\left[M_{\odot}\right]\end{array}$ \\
\hline No Dust & $391(216)$ & $5.88(5.23)$ \\
Dust & $82(25)$ & $5.59(5.14)$ \\
Best Fit & $158(100)$ & $5.57(5.26)$ \\
\hline
\end{tabular}


between truncation and steepening and we choose here to test our ages against steeper IMF slopes. The effect on our models should be that fewer massive stars are generated, resulting in less ionizing/UV emission and redder theoretical SEDs. Then our associations should be fit at younger ages.

We fit our associations with two more Starburst99 model sets with $\alpha=3.3$ (Meurer et al. 2009) and $\alpha=4.5$ for the mass range $0.5-100 M_{\odot}$. All other parameters are held constant (see Section 3). The result is consistent with the chance in IMF slope having little effect on the age determinations made previous in this study. On average, associations actually became older by $26 \mathrm{Myr}$ for $\alpha=3.3$ and $36 \mathrm{Myr}$ for $\alpha=4.5$, in the case of no dust. The case of $E(B-V)=0.3$ also resulted in a small change to older ages. As a sanity check, an IMF was generated with an extreme slope of 6.0 for a smaller range (1-400 Myr). These fits did result in much younger ages, as predicted. However, slopes this extreme have no observational evidence and are often disregarded in the literature.

The relative insensitivity of our best fit ages to the steepening of the stellar IMF slope can be understood by recalling that a steeper IMF will have the most immediate effect on the short-lived massive stars, but not on the stars contributing to the emission in the GALEX and Spitzer IRAC bands. This can be seen in Figure 23, where the spectral energy distribution of the $10 \mathrm{Myr}$ and $300 \mathrm{Myr}$ Starburst99 cluster models are plotted for a range of IMF slopes. IMF slopes up to $\alpha=4.5$ produce minimal impact on the cluster's spectral energy distribution. As an additional test, we show in Figure 24 the FUV $-3.6 \mu \mathrm{m}$ color as a function of model age for each of the four IMF slopes overplotted with the FUV-3.6 $\mu \mathrm{m}$ color for all star forming associations using the best fit ages as determined with a Kroupa IMF. The vast majority of associations occupy the space where the FUV $-3.6 \mu \mathrm{m}$ colors overlap for IMF slopes 2.3, 3.3, and 4.5. 

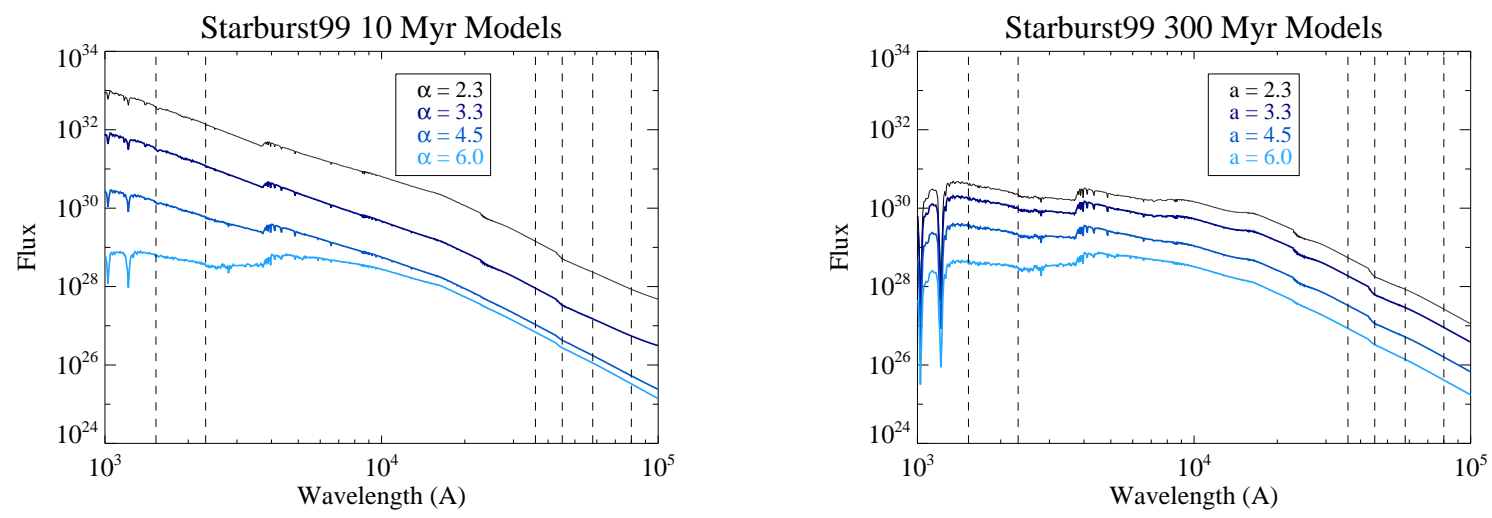

Fig. 23.- Two Starburst99 age models, $10 \mathrm{Myr}$ (left) and $300 \mathrm{Myr}$ (right), are shown for four IMF slopes: $\alpha=2.3,3.3,4.5$, and 6.0. The six dashed lines are the (from left to right) FUV, NUV, $3.6 \mu \mathrm{m}, 4.5 \mu \mathrm{m}, 5.8 \mu \mathrm{m}$, and $8.0 \mu \mathrm{m}$ bands. When using a long wavelength baseline, the age is determined by the relative strength between the UV and IR emission. For the slopes of 2.3-4.5, the ratio between UV and IR emission is relatively constant, meaning that the age of a star forming association is largely independent of the IMF slope. This is contrasted by an IMF with a slope of 6.0 , which has a very different ratio. The offset between slopes does affect the mass of the associations.

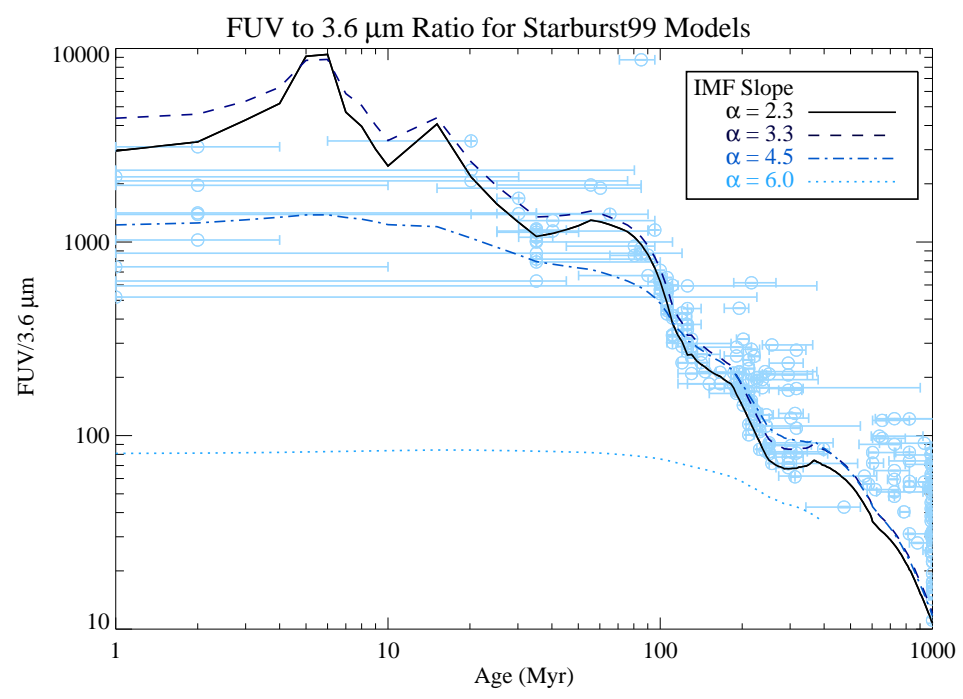

Fig. 24. - The FUV $-3.6 \mu \mathrm{m}$ color as a function of age for the sets of models with IMF slope $\alpha=2.3$ (solid), 3.3 (dashed), 4.5 (dot-dash) and 6.0 (dotted). The FUV-3.6 $\mu \mathrm{m}$ color for all associations in this study is shown in light blue for ages based on an assumed Kroupa ( $\alpha$ $=2.3)$ IMF. The typical FUV $-3.6 \mu \mathrm{m}$ error bar is smaller than the datapoints. 

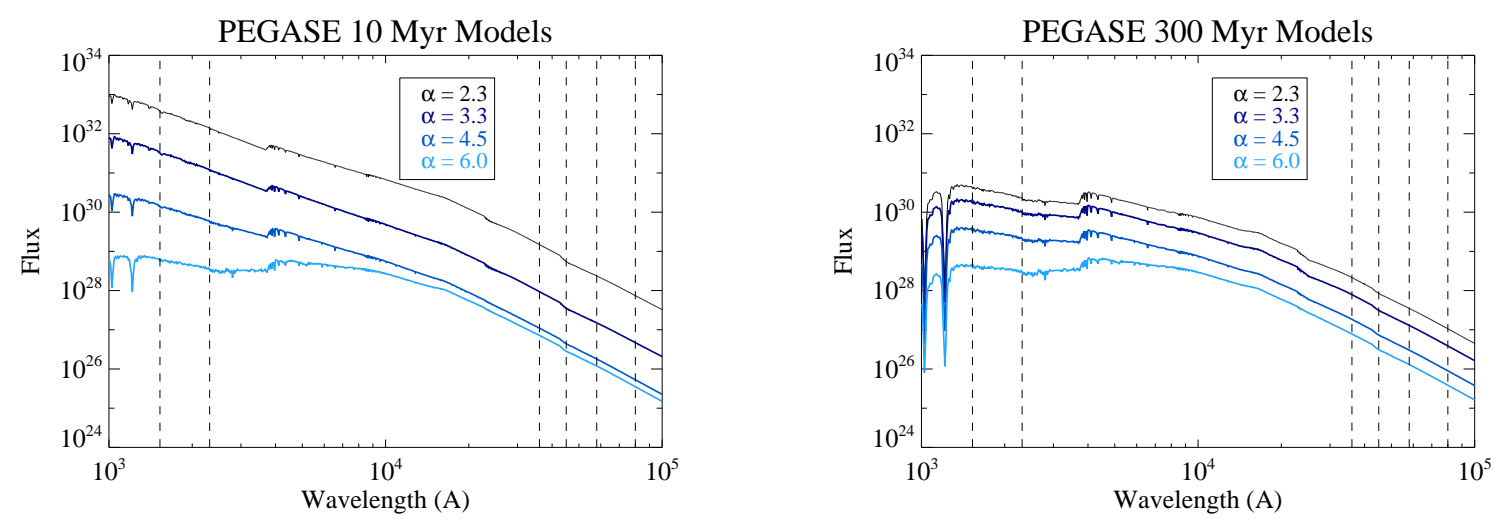

Fig. 25.- As in Figure 23, with PEGASE II models. The same effect is observed, even though the models do have subtle differences.

To further determine that this is not some systematic feature of these particular models, we also generated PEGASE II models (Fioc \& Rocca-Volmerange 1999) for the same slopes in the same mass range at the same metallicity for $10 \mathrm{My}$ and $300 \mathrm{Myr}$. This is shown in Figure 25. For both sets of models, it is clear even by eye that the ratio between the UV and the IR bands remains relatively constant from slope 2.3 to 4.5 as compared to that same ratio for a slope of 6.0. Since the age of an association is determined by the shape of the SED across these wavebands, this means that the age predicted is largely independent of the IMF slope. This in effect breaks the age-IMF degeneracy for our long wavelength baseline SEDs and tells us that, assuming we correctly account for extinction and have accurate models (the two models, Starburst99 and PEGASE II, shown here will give different age results due to differences in the predicted MIR bands), we are looking at the real age distribution. Of course, just because we cannot distinguish between IMF slopes does not mean that a top-light IMF does not exist in these regions. Future studies to quantify the dearth of $\mathrm{H} \alpha$ relative to UV and an accurate age distribution can be used to constrain IMF properties. At this point, however, while a non-standard IMF cannot be excluded, it is not required to account for the observed properties of our associations. The dearth of $\mathrm{H} \alpha$ emission in the outer disk regions is well accounted for by the spread in ages of star forming associations. That some associations are as old as, or exceed, 1 Gyr of age, suggests that the original triggering mechanism of the in-situ star formation has caused sufficient instability in the outer disk that gas clouds are still effectively collapsing and forming stars to the present time. 


\section{Conclusions and Future Work}

In this paper, we have leveraged Spitzer IRAC imaging against the GALEX FUV and NUV wavebands to determine the ages and masses of star forming associations in the lowdensity XUV disks of five nearby spiral galaxies. These associations likely consist of a few stellar clusters with typical cumulative masses of $\sim 10^{5.3} M_{\odot}$. The age dispersion across all five disks is large, spanning the range $1 \mathrm{Myr}-\gtrsim 1 \mathrm{Gyr}$, with a median age of $\sim 100$ Myr. Though the age determinations can vary considerably for different extinction values, the wide age dispersion and the old median age are robust against both extinction (in the range $E(B-V)=0-0.3 \mathrm{mag})$ and steepening of the IMF slope. This indicates that the age dispersion is real and sufficient to explain the observed dearth of $\mathrm{H} \alpha$ in XUV disks as compared to UV emission without the need to invoke a non-standard IMF.

Our insensitivity to the IMF slope is potentially useful in constraining the IMF properties in these regions. The next step in this study is to obtain deep $\mathrm{H} \alpha$ imaging of these

regions, analogous to Goddard et al. (2010), which can be used to confirm our results and give us further insight into the youngest modes of star formation in these low-density regions. In addition, optical datapoints would allow us to further constrain the ages of these associations. In particular, the $\mathrm{U}-\mathrm{B}$ color is highly sensitive to the age of an association while being relatively insensitive to extinction. Combined, $\mathrm{H} \alpha$ observations and accurate ages would provide a powerful diagnostic tool for studies of the local IMF.

This work has made use of the Spitzer Space Telescope data obtained as part of the Cycle 3 GO program 30753. It has been partially supported by the JPL, Caltech, Contract Number 1296193. This research has made use of the NASA/IPAC Extragalactic Database (NED) which is operated by the Jet Propulsion Laboratory, California Institute of Technology, under contract with the National Aeronautics and Space Administration.

\section{REFERENCES}

Bertin, E., Arnouts, S., 1996, A\&AS, 117, 393

Bianchi, L., Madore, B., Thilker, D., et al., 2003, AAS, 203, 9112

Bianchi, L., 2009, ApSS, 320, 11

Boissier, S., Gil de Paz, A., Boselli, A., et al, 2007, ApJS, 173, 524

Bresolin, F., Ryan-Weber, E., Kennicutt, R. C., Goddard, Q., 2009, ApJ, 695, 580

Calzetti, D., Armus, L., Bohlin, R., et al., 2000, ApJ, 533, 682 
Calzetti, D., Kennicutt, R., Bianchi, L., et al., 2005, ApJ, 633, 871

Calzetti, D., Kennicutt, R. C., Engelbract, C., et al., 2007, ApJ, 666, 870

Cuillandre, J., Lequeux, J., Allen, R. J., Mellier, Y., Bertin, E., 2001, ApJ, 554, 190

Dale, D. A., Cohen, S. A., Johnson, L. C., et al. 2009, ApJ, 703, 517

Draines, B. T., Dale, D. A., Bendo, G., et al. 2007, ApJ, 663, 866

Dong, H., Calzetti, D., Regan, M., Thilker, D., Bianchi, L., Meurer, G.R., \& Walter, F., 2008, AJ, 136, 479

Engelbracht, C. W., Gordon, K. D., Rieke, G. H., et al., 2005, ApJ, 628, L29

Engelbract, C. W., Rieke G. H., Gordon, K. D., et al., 2008, ApJ, 678, 804

Fall, S. M., Chandar, R., Whitmore, B. C., 2005, ApJ, 631, 133

Fazio, G. G., Hora, J. L., Allen, L. E., 2004, ApJS, 154, 10

Ferguson, A. M. N., Gallagher, J. S., Wyse, R. F. G., 1998, AJ, 116, 673

Fioc, M., Rocca-Volmerange, B., 1999, arXiv:astro-ph/9912179v1

Gil de Paz, A., Madore, B. F., Boissier, S., et al., 2005, ApJ, 627, L29

Gil de Paz, A., Madore, B. F., Boissier, S., et al., 2007, ApJ, 661, 115

Gil de Paz, A., Boissier, S., Madore, B. F., et al., 2007, ApJS, 173, 185

Goddard, Q. E., Kennicutt, R. C., \& Ryan-Weber, E. V., 2010, MNRAS, 405, 2781

Hunter, D. A., Elmegreen, B. G., Dupuy, T. J., et al., 2003, AJ, 126, 1836

Kennicutt, R. C., 1989, ApJ, 344, 685

Kennicutt, R. C., 1998, ApJ, 498, 541

Kennicutt, R. C., 1998, ARA\&A, 36, 189

Kennicutt, R. C., Armus, L., Bendo, G., Calzetti, D., et al., 2003, PASP, 115, 928

Kroupa, P., 2001, MNRAS, 322, 231

Leger, A., \& Puget, J. L., 1984, A\&A, 137, 5 
Leitherer, C., Schaerer, D., Goldader, J. D., et al., 1999, ApJS, 123, 3

Lelievre, M., Roy, J., 2000, AJ, 120, 1306L

Maiz-Apellaniz, J., 2001, ApJ, 563, 151

Maraston, C., Daddi, E., Renzini, A., et al., 2006, ApJ, 652, 85

Martin, C. L., \& Kennicutt, R. C., 2001, APJ, 555, 301

Meurer, G., Wong, O. I., Kim, J. H., et al., 2009, ApJ, 695, 765

Morrissey, P., Conrow, T., Barlow, T. A., et al., 2007, ApJS, 173, 682

Mun̈oz-Mateos, J. C., Gil de Paz, A., Boissier, S., Zamorano, J., Jarrett, T., Gallego, J., Madore, B. F., 2007, ApJ, 658, 1006

Pahre, M., Ashby, M. L. N., Fazio, G. G., Willner, S. P., 2004, ApJS, 154, 229

Pflamm-Altenburg, Wiender, C., Kroupa, P., 2007, ApJ, 671, 1550

Roskar, R., Debattista, V. P., Stinson, G. S., et al., 2008, ApJ, 675, L65

Schlegel, D. J., Finkbeiner, D. P., Davis, M., 1998, ApJ, 500, 525

Smith, J. D. T., Draine, B. T., Dale, D. A., et al., 2007, ApJ, 656, 770

Thilker, D. A., Bianchi, L., Boissier, S., et al., 2005, ApJ, 619, L79

Thilker, D. A., Bianchi, L., Meurer, G., et al., 2007, ApJS, 173, 538

Vazquez, G. A., \& Leitherer, C., 2005, ApJ, 621, 695

Weidner, C., \& Kroupa, P., 2004, MNRAS, 248, 187

Weidner, C., \& Kroupa, P., 2005, ApJ, 625, 754

Werk, J. K., Putman, M. E., Meurer, G. R., et al., 2010, ApJ, 715, 656

Wyder, T. K., Treyer, M. A., Milliard, B., et al., 2005, ApJ, 619, 15

Xu, C. K., Donas, J., Arnouts, S., et al. 2005, ApJ, 619, L11

Zaritsky, D., \& Christlein, D., 2007, AJ, 134, 135 